\title{
UPPER AND LOWER VISUAL FIELD DIFFERENCES IN PERCEPTUAL ASYMMETRIES
}

\author{
A Thesis Submitted to the College of \\ Graduate Studies and Research \\ In Partial Fulfillment of the Requirements \\ For the Degree of Doctor of Philosophy \\ In the Department of Psychology \\ University of Saskatchewan \\ Saskatoon
}

By

NICOLE THOMAS

(C) Copyright Nicole Annette Marie Thomas, December, 2010. All rights reserved. 


\section{Permission to Use}

In presenting this thesis in partial fulfilment of the requirements for a Postgraduate degree from the University of Saskatchewan, I agree that the Libraries of this University may make it freely available for inspection. I further agree that permission for copying of this thesis in any manner, in whole or in part, for scholarly purposes may be granted by the professor or professors who supervised my thesis work or, in their absence, by the Head of the Department or the Dean of the College in which my thesis work was done. It is understood that any copying or publication or use of this thesis or parts thereof for financial gain shall not be allowed without my written permission. It is also understood that due recognition shall be given to me and to the University of Saskatchewan in any scholarly use which may be made of any material in my thesis.

Requests for permission to copy or to make other use of material in this thesis in whole or part should be addressed to:

Head of the Department of Psychology

University of Saskatchewan

Saskatoon, Saskatchewan S7N 5A5 


\begin{abstract}
Neurologically normal individuals show a leftward spatial bias and tend to collide with objects on the right side more frequently than on the left. The upper visual field is associated with extrapersonal space, and mediated by the ventral stream through parvocellular projections. The lower visual field is associated with peripersonal space, and mediated by the dorsal stream through magnocellular projections. Upper and lower visual field differences have been observed in perceptual asymmetries but results have been mixed. Object- and space-based coordinates also both influence the leftward bias; however their relative contributions are unknown as similar spatial conditions are often collapsed across. More left-side collisions emerged on a route following task in the lower visual field and more right-side collisions were seen in the upper visual field (Thomas, Stuckel, Gutwin, \& Elias, 2009). Left-handers made more right-side collisions in the central condition, whereas right-handers showed no bias. Leftward biases on the greyscales task were stronger in the lower visual field; however no distance-based differences were observed (Thomas \& Elias, 2010). A stronger spatial bias was found on the greyscales task, whereas a stronger object-based bias was found on the object luminosity task (Thomas \& Elias, in press). When individual spatial conditions were examined, the image chosen most often was always located in the lower field. Stimulus type and spatial location interacted to determine which coordinate type contributes more strongly to leftward biases. We also found that the leftward bias on the greyscales task was stronger in the lower visual field during prolonged presentation and in the upper visual field during brief presentation. A global motion task was created to preferentially engage magnocellular projections to the dorsal stream. Isoluminant red/green and blue/yellow colour tasks, which preferentially engage parvocellular projections to the ventral stream, were also created. Leftward biases were seen on the greyscales and motion tasks. On an isoluminant colour task, biases were significantly weakened, suggesting leftward
\end{abstract}


biases exhibited by neurologically normal people are mediated by magnocellular projections to the dorsal stream and this preferential processing leads to a lower visual field advantage on the greyscales task. 


\section{ACKNOWLEDGMENTS}

I am extremely grateful to the many people who made this dissertation possible. This work would not have been possible without the continual support of my graduate studies supervisor, Dr. Lorin Elias. His enthusiasm for both research and teaching were very inspirational and allowed me to appreciate the positive aspects of both components of academia. He provided me with an excellent example of how to be a good and ethical scientist as well as a professional in my field.

I am thankful for the support given to me by my advisory committee throughout the course of my research. I sincerely appreciate the assistance and feedback provided to me by Dr. Carl Gutwin, Dr. Ron Borowsky, and Dr. Mirna Vrbancic, which helped me greatly in completing my research. I am also thankful for the technical assistance provided to me by Dr. Gutwin and his students Dane Stuckel and Oliver Schneider. The computer programming that they assisted me with was instrumental to my research. I am also grateful to all the past members of the Elias lab for the assistance and support that they gave to me during my time there.

I gratefully acknowledge the financial supported provided to me by the Natural Sciences and Engineering Research Council throughout my undergraduate and graduate careers. The scholarships I received provided me with many opportunities that helped me with the work completed for this dissertation.

I would like to thank my parents, Dave and Suzanne Thomas, for the ongoing support that they provided to me while I was completing my dissertation. They raised me to believe in the importance of education and independence, which motivated me to complete my Ph.D. I would also like to thank my friends and fellow graduate students for the support that they gave me throughout my graduate studies. Lastly, I would like to thank my boyfriend, Jerrod Weisbrod, for always being there for me, for caring and for supporting me when I needed it the most. 


\section{TABLE OF CONTENTS}

page

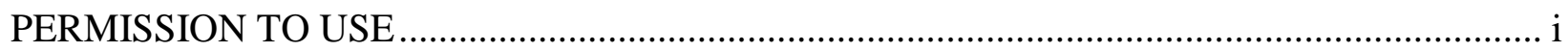

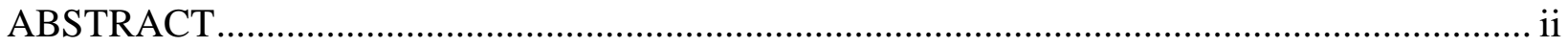

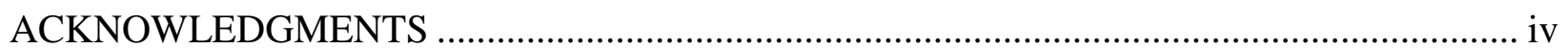

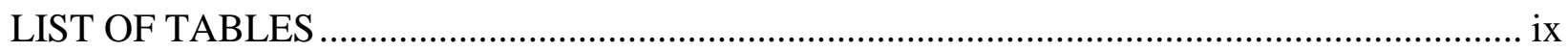

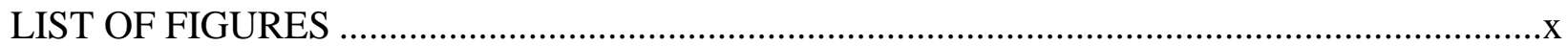

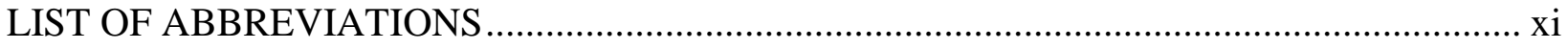

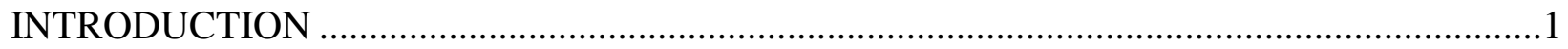

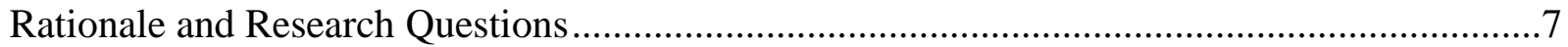

DIRECTIONAL COLLISIONS DURING A ROUTE FOLLOWING TASK ..........................10

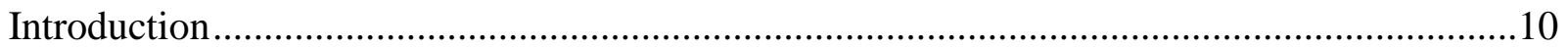

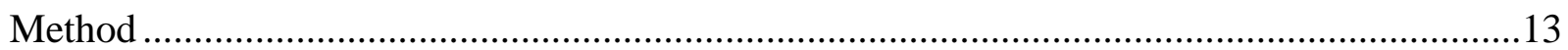

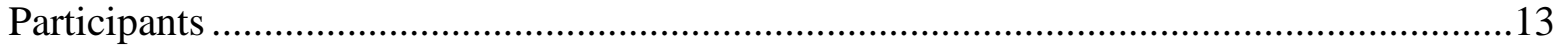

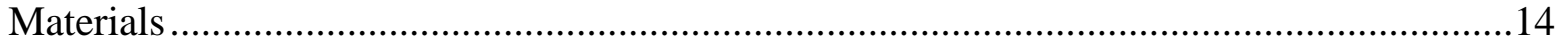

Waterloo Handedness Questionnaire-Revised.........................................................14

Computer-based Route Following Task .................................................................14

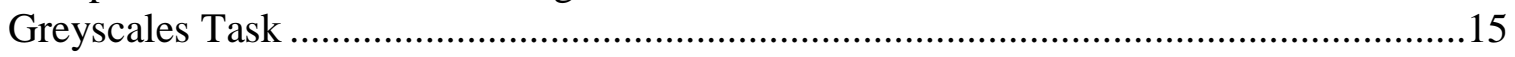

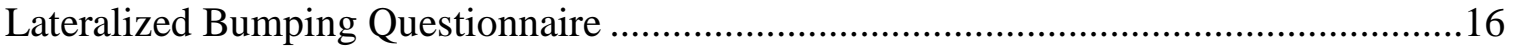

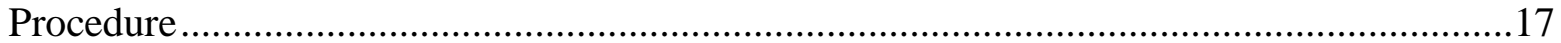

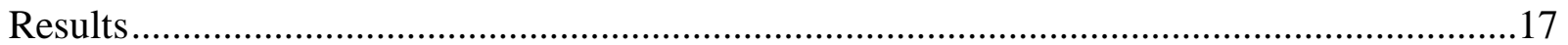

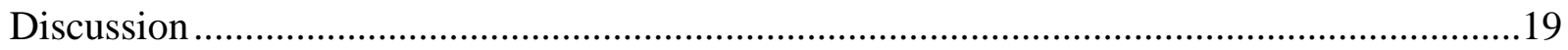

DO PERCEPTUAL ASYMMETRIES DIFFER IN PERIPERSONAL AND

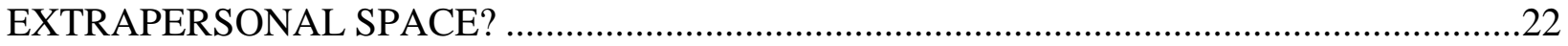

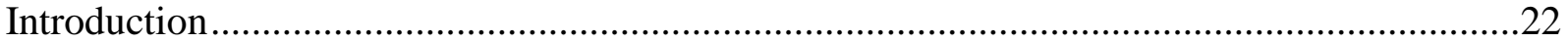

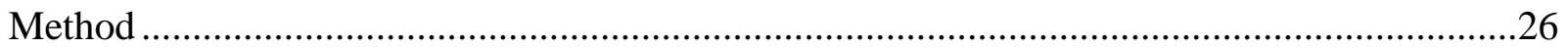

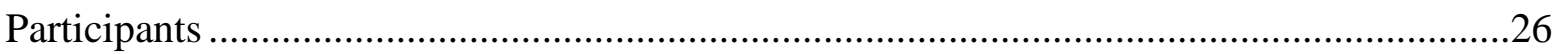

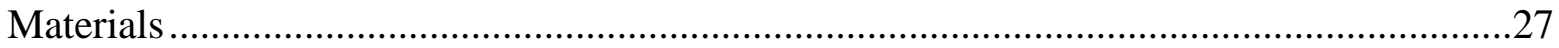

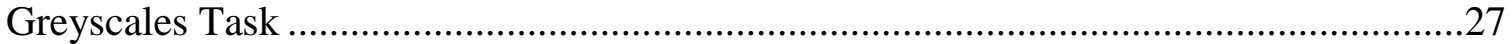

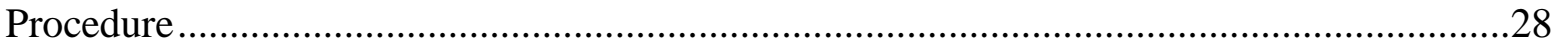

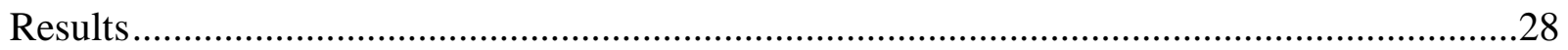

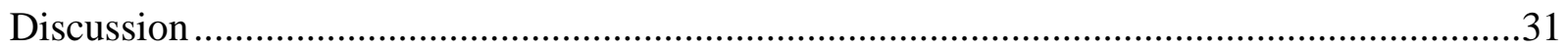

PERCEPTUAL ASYMMETRIES IN GREYSCALES: OBJECT-BASED VERSUS SPACE-

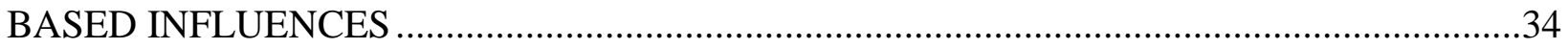

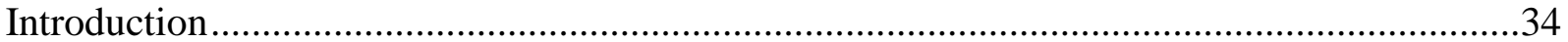

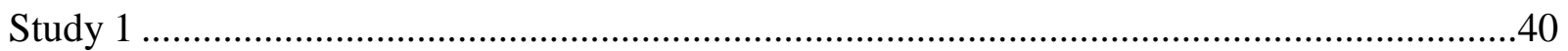

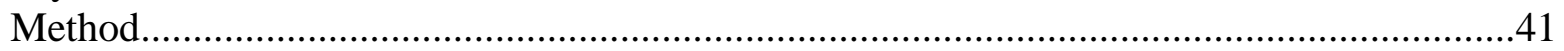




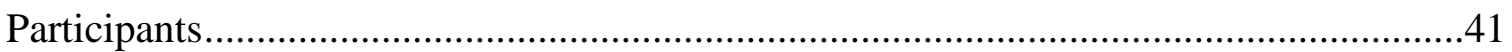

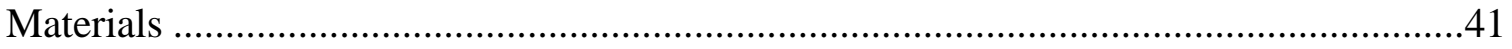

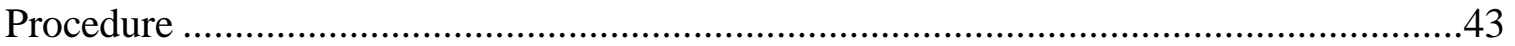

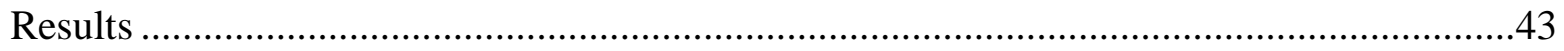

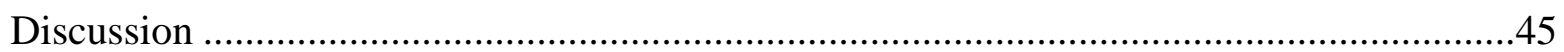

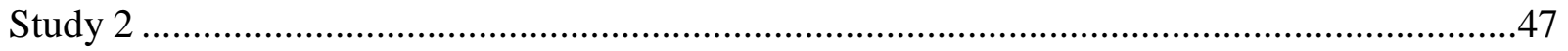

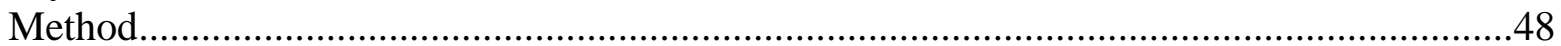

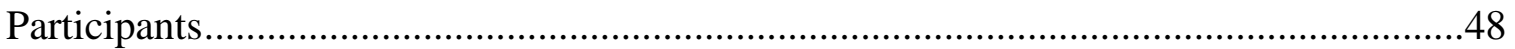

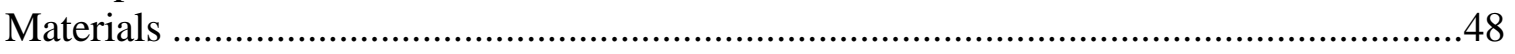

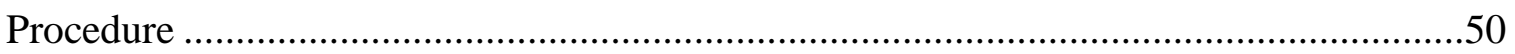

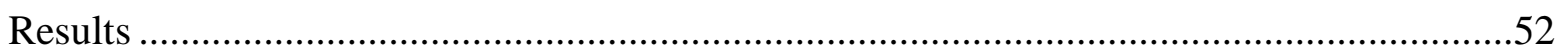

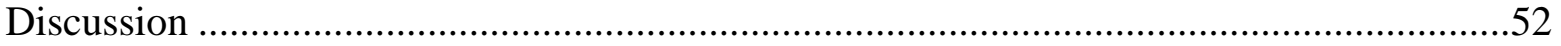

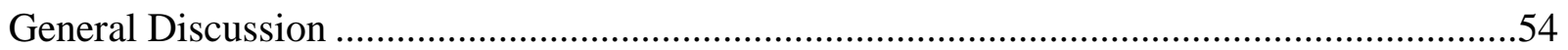

UPPER AND LOWER VISUAL FIELD DIFFERENCES IN PERCEPTUAL

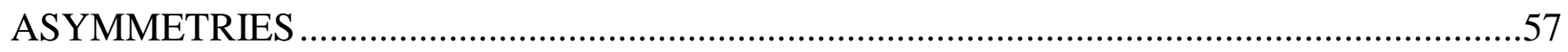

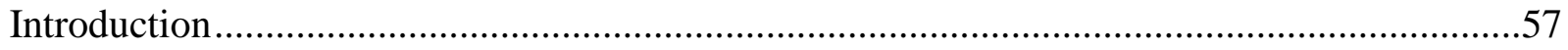

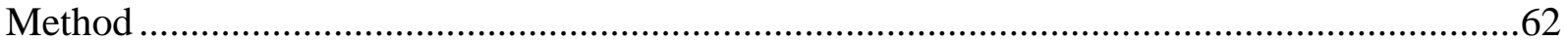

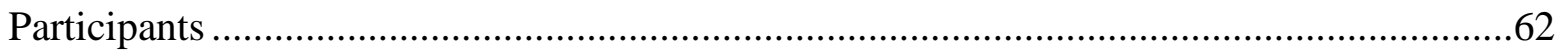

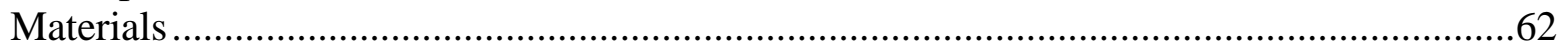

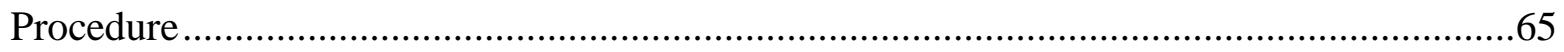

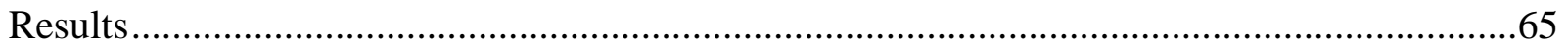

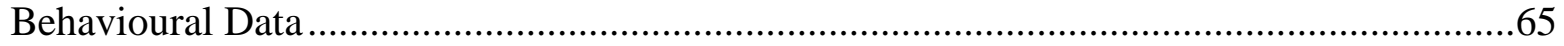

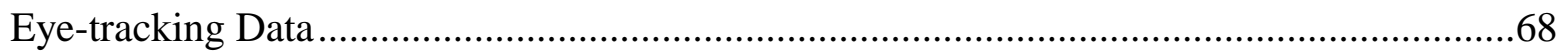

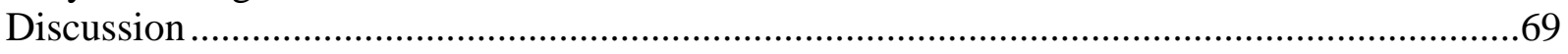

DORSAL AND VENTRAL STREAM CONTRIBUTIONS TO PERCEPTUAL

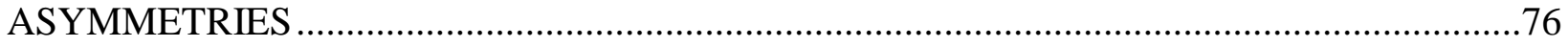

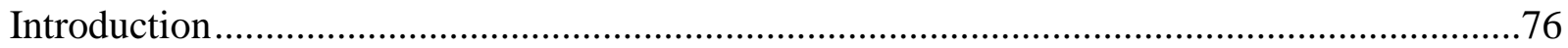

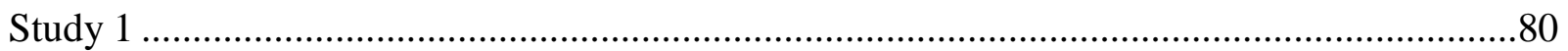

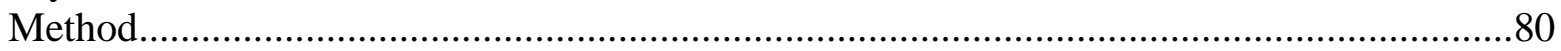

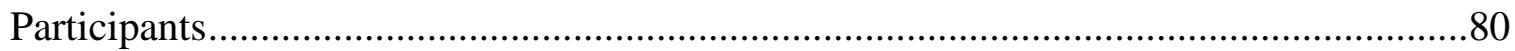

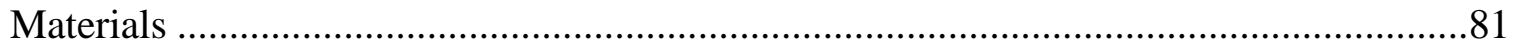

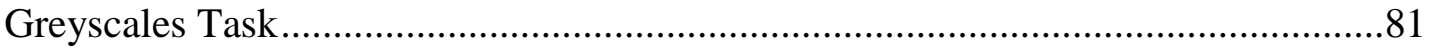

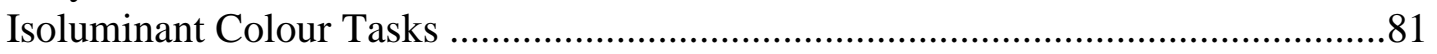

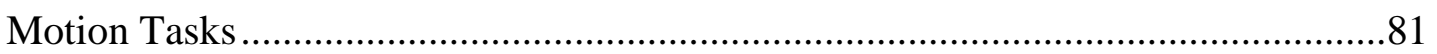

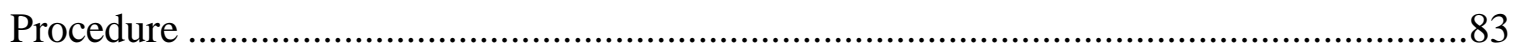

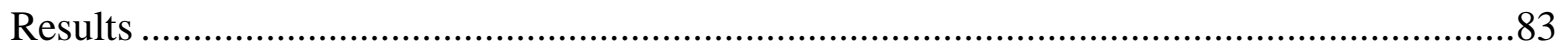

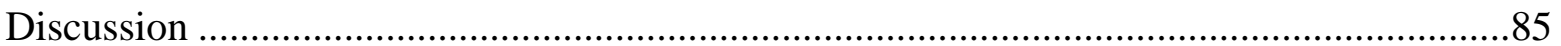

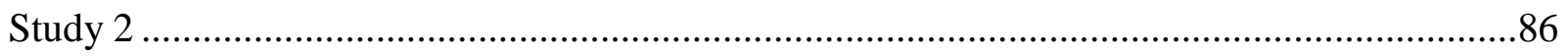

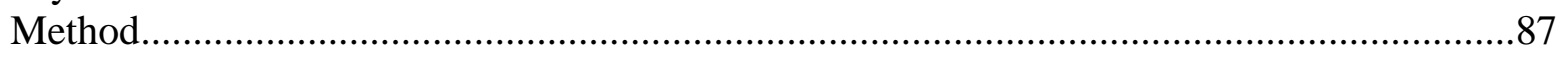

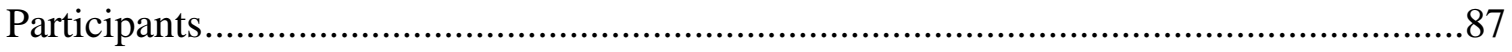

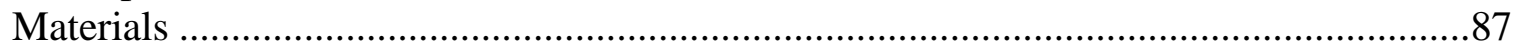

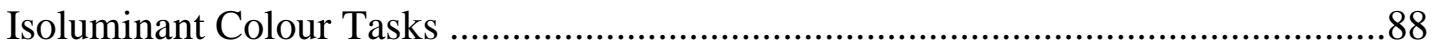

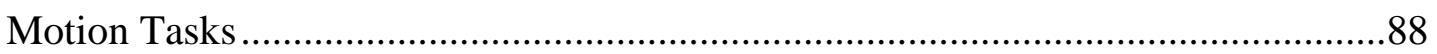

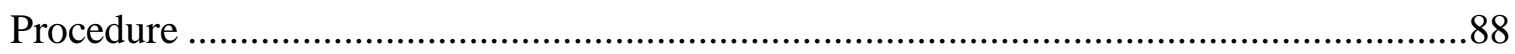

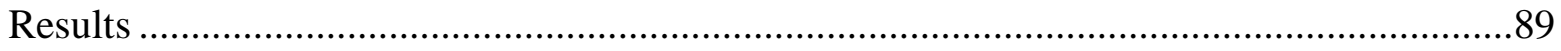




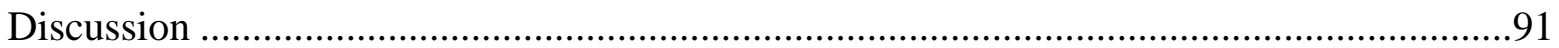

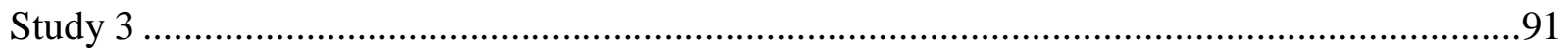

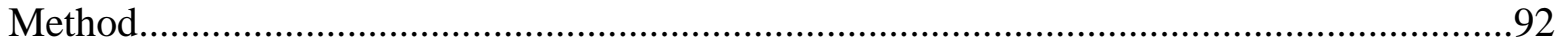

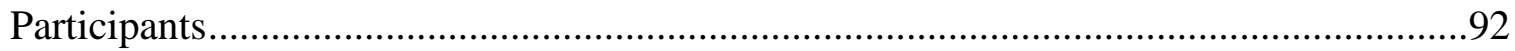

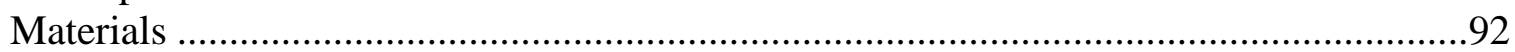

Isoluminant Colour Tasks …………………………......................................93

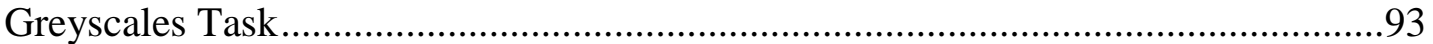

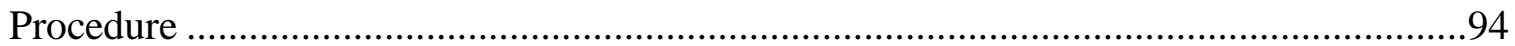

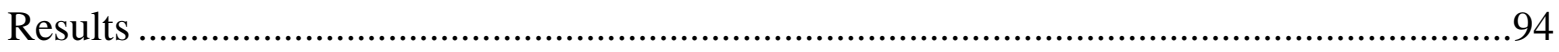

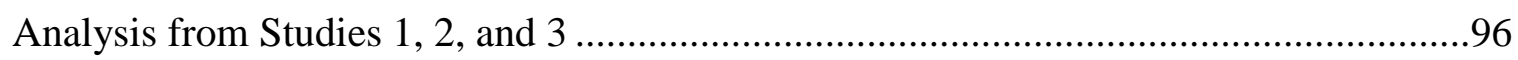

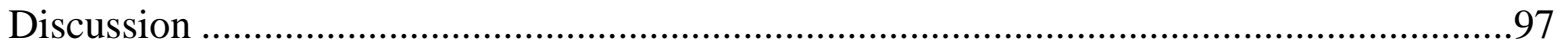

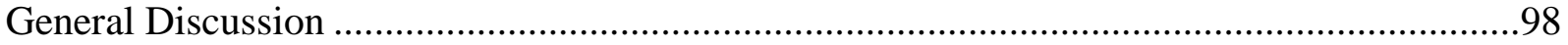

DISCUSSION, CONCLUSIONS AND FUTURE RESEARCH...............................................101

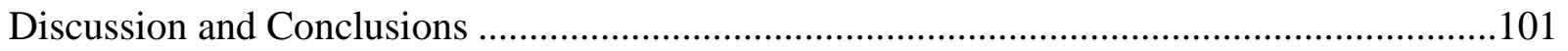

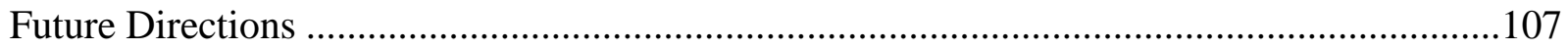

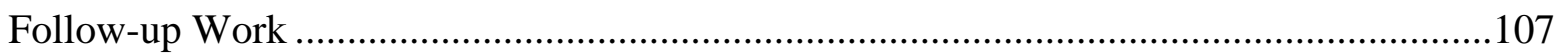

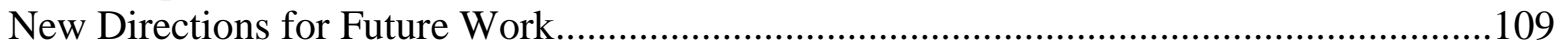

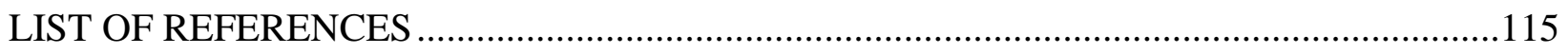

WATERLOO HANDEDNESS AND FOOTEDNESS QUESTIONNAIRE-REVISED.............134

STARTING VIEW OF THE COMPUTER-BASED ROUTE FOLLOWING TASK.................136

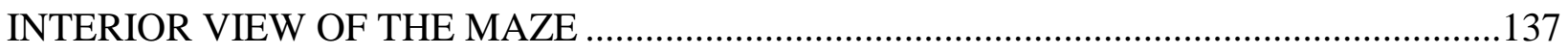

SAMPLE STIMULI FROM THE GREYSCALES TASK ……………………………........138

BUMPING QUESTIONNAIRE FROM DIRECTIONAL COLLISIONS DURING A ROUTE

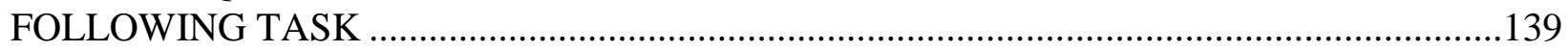

CONSENT FORM FROM DIRECTIONAL COLLISIONS DURING A ROUTE FOLLOWING

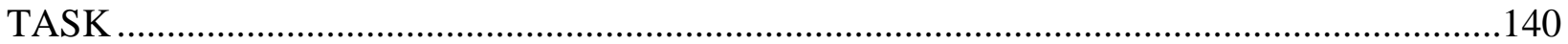

DEBRIEFING FORM FROM DIRECTIONAL COLLISIONS DURING A ROUTE

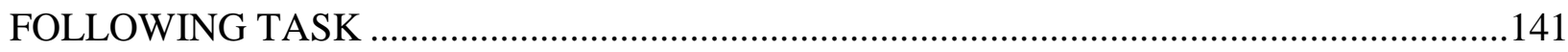

SAMPLE STIMULI FROM THE GREYSCALES TASK WHEN PRESENTED TO EITHER

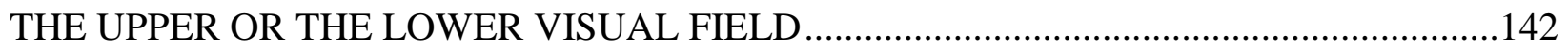

CONSENT FORM FROM DO PERCEPTUAL ASYMMETRIES DIFFER IN

PERIPERSONAL AND EXTRAPERSONAL SPACE?

DEBRIEFING FORM FROM DO PERCEPTUAL ASYMMETRIES DIFFER IN PERIPERSONAL AND EXTRAPERSONAL SPACE? . 
SUMMARY OF PRESENTATION CONDITIONS PREVIOUSLY EMPLOYED IN PERCEPTUAL ASYMMETRIES STUDIES COMPARING OBJECT- AND SPACE-BASED INFLUENCES .

SUMMARY OF PRESENTATION CONDITIONS USED IN PERCEPTUAL

ASYMMETRIES IN GREYSCALES: OBJECT-BASED VERSUS SPACE-BASED

INFLUENCES (STUDY 1)....

CONSENT FORM FROM PERCEPTUAL ASYMMETRIES IN GREYSCALES: OBJECTBASED VERSUS SPACE-BASED INFLUENCES (STUDY 1) AND UPPER AND LOWER VISUAL FIELD DIFFERENCES IN PERCEPTUAL ASYMMETRIES

DEBRIEFING FORM FROM PERCEPTUAL ASYMMETRIES IN GREYSCALES: OBJECTBASED VERSUS SPACE-BASED INFLUENCES (STUDY 1).

STIMULI USED IN PERCEPTUAL ASYMMETRIES IN GREYSCALES: OBJECT-BASED VERSUS SPACE-BASED INFLUENCES (STUDY 2)

CONSENT FORM FROM PERCEPTUAL ASYMMETRIES IN GREYSCALES: OBJECT-

BASED VERSUS SPACE-BASED INFLUENCES (STUDY 2).

DEBRIEFING FORM FROM PERCEPTUAL ASYMMETRIES IN GREYSCALES: OBJECTBASED VERSUS SPACE-BASED INFLUENCES (STUDY 2).

DEBRIEFING FORM FROM UPPER AND LOWER VISUAL FIELD DIFFERENCES IN PERCEPTUAL ASYMMETRIES.

SAMPLE STIMULI FROM THE RED-GREEN ISOLUMINANT COLOUR TASK .153

CONSENT FORM FROM DORSAL AND VENTRAL STREAM CONTRIBUTIONS TO PERCEPTUAL ASYMMETRIES.

DEBRIEFING FORM FROM DORSAL AND VENTRAL STREAM CONTRIBUTIONS TO PERCEPTUAL ASYMMETRIES. 


\section{LIST OF TABLES}

$\underline{\text { Table }}$

page

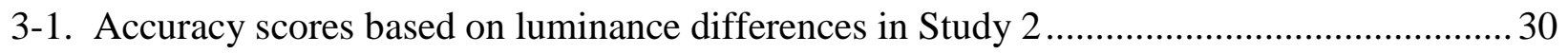

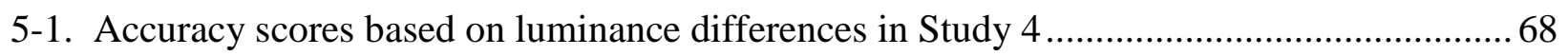

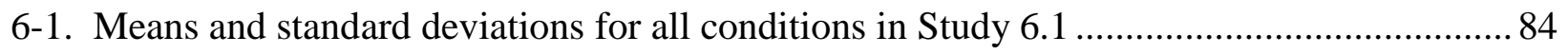

6-2. Means and standard deviations for all conditions in Study 6.2 ................................... 90

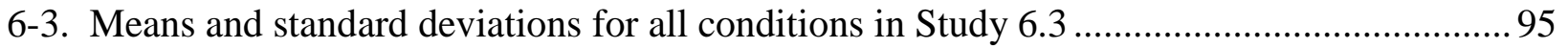




\section{LIST OF FIGURES}

$\underline{\text { Figure }}$

page

1-1. Description of the different ways in which visual field differences were examined in the current research program. The first stream examined processing differences by comparing space-based differences in peripersonal vs. extrapersonal space. The second stream examined spatial location by comparing upper and lower visual field differences directly as well as their relation to the left and right visual field differences.................................................................

2-1. Handedness by presentation condition interaction. Values represent mean bias scores obtained by subtracting the number of leftward collisions from the number of rightward collisions, with positive values indicating a rightward bias and negative values a left side bias. Error bars represent the standard errors of the mean

2-2. Scatterplot illustrating the lack of relationship between the greyscales task and each of the conditions of the route following task

3-1. Interaction of visual field and viewing distance. Error bars represent standard errors of the mean.....

4-1. Interaction of horizontal shift and relative position. Error bars indicate the standard error of the mean

4-2. Interaction of horizontal shift and vertical location. Error bars indicate the standard error of the mean

4-3. Interaction of side of space and side of luminosity. Error bars indicate the standard error of the mean

5-1. Interaction of presentation time and visual field. Error bars indicate the standard error of the mean.

6-1. Mean bias scores for motion, luminance, red and green tasks completed in Experiment 1 of Study 5. Error bars indicate the standard error of the mean

6-2. Mean bias scores for free-viewing and non-free-viewing motion as well as red and green tasks completed in Experiment 2 of Study 5. Error bars indicate the standard error of

the mean

6-3. Mean bias scores for blue and yellow tasks, non-free-viewing green and red tasks and the greyscales task completed in Experiment 3 of Study 5. Error bars indicate the standard error of the mean

6-4. Mean bias scores for colour, motion, luminance and red tasks across all three experiments

in Study 5. Error bars indicate the standard error of the mean 


\section{LIST OF ABBREVIATIONS}

$\underline{\text { Abbreviation }}$

page

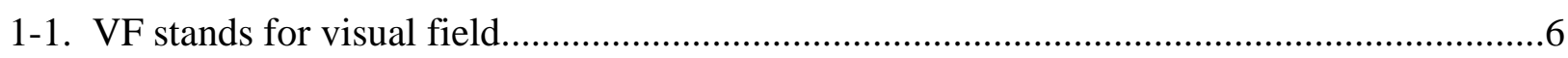

2-1. ANOVA stands for analysis of variance ............................................................ 15

4-1. TL/BR stands for top left/bottom right pairing ................................................... 49

4-2. BL/TR stands for bottom left/top right pairing ...................................................... 49

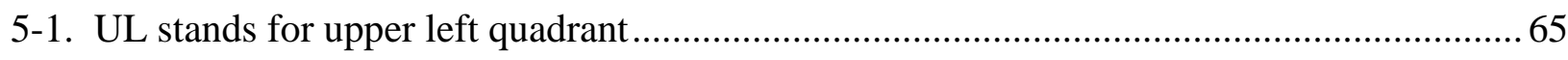

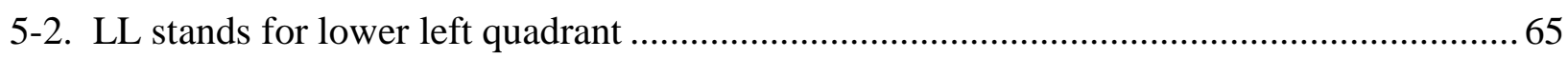

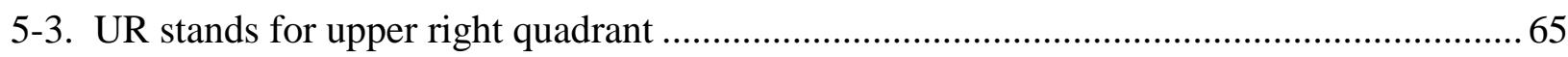

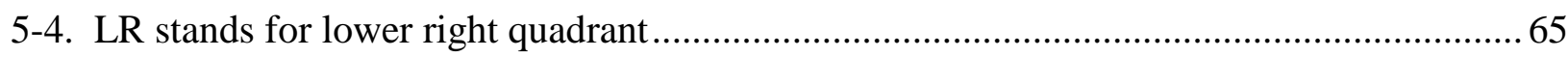




\section{CHAPTER 1}

\section{INTRODUCTION}

In 1941, Brain described a previously unfamiliar deficit. Following a right parietal lesion, three patients were unable to follow familiar routes. His patients were making right turns instead of left turns, leading them to get lost and disoriented in their own homes. Further, these patients reported frustration as they were aware that they had made a mistake, but could not describe what the error was. Interestingly, the patients were able to correctly visualize the route and to verbally describe how to get to each location. Brain (1941) referred to this navigational disturbance as neglect of the left side of space.

"The effect of a lesion of the posterior part of the right hemisphere is to cause the patient to neglect the left half of external space and to select a right turn instead of a left turning, or even the right hand of two doors or other objects facing him, in error" (Brain, 1941, p. 263). Brain (1941) goes on to describe the patients' attitude toward the left side of extrapersonal space as similar to that observed in patients with a disordered perception of the left side of their own body. He concludes that, "the patients attitude to the left half of external space was one of inattention or neglect: in walking he behaved as if it were not there, but in conversation he recognized its existence” (Brain, 1941, p. 264). Brain's (1941) description of these patients would forever change the way in which visual inattention was studied, as hemispatial neglect research was born from it.

Neglect is most commonly defined as an inability to identify or orient to information or objects on the side contralateral to a brain lesion, a failure that neither sensory nor motor defects can account for (Heilman, 1979). Early reports of neglect-like behaviours were made by several German researchers, including Anton, Pick, Poppelreuter and Zingerle (see Halligan \& Marshall, 1993 for a review), as well as by Holmes (1918; 1919), with the earliest account believed to be 
that described by Hughlings Jackson in 1876 (Halligan \& Marshall, 1993). It is thought that Pineas was the first to consistently use the term neglect in his description of a 60-year-old woman who did not have any field deficits or sensorimotor loss, but demonstrated neglect of the left side (Halligan \& Marshall, 1993). Interestingly, it would be 65 years after these early descriptions of the behavioural features that would eventually come to be associated with hemispatial neglect, that Brain (1941) first reported the precise characteristics that isolated visual neglect as a distinct disorder and identified it as a disturbance of perceptual space. As a result of this, Brain is credited with changing the way in which neglect was studied.

The primary conclusions made by Brain (1941) indicated that neglect was neither a visual field (VF) nor a sensory deficit, but was related to posterior lesions in the right hemisphere. Previous work had used the terms visual inattention and neglect interchangeably and Brain (1941) identified that there was a difference between the two, and further, that neglect should be distinguished from topographical memory loss, visual agnosia and left-right discrimination problems. Zangerwill and colleagues followed up on the work of Brain (1941), completing a great deal of research between 1944 and 1960 (Halligan \& Marshall, 1993). They were the first to illustrate that neglect could be dissociated based on distance, occurring solely in peripersonal or extrapersonal space. Between the years of 1970 and 1990, a drastic increase in the number of studies looking at neglect was seen (Halligan \& Marshall, 1993). This work isolated many different contexts in which neglect could occur, including further dissociations based on spatial coordinate systems, including personal, peripersonal, extrapersonal and representational space. It was also shown that deficits could occur in three separate frames of reference: environmentcentered, body-centered (i.e., retinal, head, and trunk), and object-centered (Halligan \& Marshall, 1993). 
As work looking at hemispatial neglect began to grow in popularity, Bowers and Heilman (1980) decided to examine tactile line bisection performance in neurologically normal participants. This was done in an attempt to determine whether laterality effects were the result of a hemispheric mechanism involved in perceiving and acting in the contralateral hemispace or whether these effects were related to hemispheric processing specialization. Bowers and Heilman (1980) found that participants were more likely to bisect to the left of centre, regardless of whether they employed their left hand or their right hand and whether the bisection was in the right hemispace or the left hemispace or at the midline. This type of directional error had not been demonstrated previously and Bowers and Heilman (1980) termed the phenomena pseudoneglect, as it resembled the directional biases of clinical hemispatial neglect patients on line bisection tasks.

Spatial biases have subsequently been observed on several measures, including both manual and computerized line bisection (i.e., Jewell \& McCourt, 2000; McCourt \& Jewell, 1999), cancellation tasks (Vingiano, 1991), the landmark task (Dufour, Touzalin, \& Candas, 2007), the greyscales task (Nicholls, Bradshaw, \& Mattingley, 1999), and measures looking at judgments of numerosity, size (Nicholls et al., 1999) and distance (Krupp, Robinson, \& Elias, in press). Lateral biases have also been identified on more real-world behaviours, such as passing through a doorway (Nicholls, Loftus, Mayer, \& Mattingley, 2007; Nicholls, Loftus, Orr, \& Barre, 2008), choosing a seat in the theatre (Okubo, 2010), completing a route following task (Thomas, Stuckel, Gutwin, \& Elias, 2009) or multiple-choice questionnaire response patterns (Nicholls, Orr, Okubo, \& Loftus, 2006). The leftward lateral biases seen on laboratory measures also occur on more real-world types of tasks, making it of extreme importance to understand more clearly how and why these biases emerge. 
As the left bias is likely due to a bias in spatial attention (i.e., Bjoertomt, Cowey, \& Walsh, 2002; Çiçek, Deouell, \& Knight, 2009; Corbetta, Shulman, Miezin, \& Petersen, 1995; Fink et al., 2000; Fink, Marshall, Weiss, \& Zilles, 2001; Foxe, McCourt, \& Javitt, 2003; Posner \& Petersen, 1990; Posner \& Rothbart, 2007), the influence of spatial location has been investigated. This has typically been done by isolating stimuli to the right VF or the left VF, to avoid having information processed in both VFs. Although several studies have examined this factor in line bisection, no consistent findings have emerged. There have been reports of a rightward bias occurring when lines are presented in the right hemispace and a leftward bias when lines are in the left hemispace (McCourt, Garlinghouse, \& Slater, 2000; McCourt \& Jewell, 1999; Milner, Brechmann, \& Pagliarini, 1992; Reuter-Lorenz, Kinsbourne, \& Moscovitch, 1990). However, bisections have also been in the opposite direction of the presented hemispace (McCourt et al., 2000; Nichelli, Rinaldi, \& Cubelli, 1989). Further complicating matters, some researchers have observed exclusively leftward (Luh, 1995) or rightward biases (Mennemeier, Vezey, Chatterjee, Rapcsak, \& Heilman, 1997; Nielsen, Intriligator, \& Barton, 1999) under both VF conditions. A meta-analysis by Jewell and McCourt (2000) identified a pattern whereby bisections were most commonly leftward in the left hemispace and rightward in the right hemispace, but results have generally been inconsistent.

An upward bias has been observed when using vertical stimuli, that span both the upper and lower VFs (Bradshaw, Nettleton, Nathan, \& Wilson, 1985; Drain \& Reuter-Lorenz, 1996; McCourt \& Olafson, 1997; Nicholls, Mattingley, Berberovic, Smith, \& Bradshaw, 2004; Nicholls, Smith, Mattingley, \& Bradshaw, 2006). Biases toward the upper features have been observed on line bisection (Bradshaw et al., 1985; Drain \& Reuter-Lorenz, 1996; McCourt \& Olafson, 1997) and on the greyscales task (Nicholls et al., 2004b; 2006b). Both leftward and 
upward biases have been explained in relation to object-centred biases in that the most significant features of a stimulus are attended to. Left side features are likely significant due to Western reading and scanning habits and the upper features of a stimulus are thought to carry more information than the lower ones (Jeerakathil \& Kirk, 1994). This has been demonstrated by showing a bias toward the label "top", even when the labelled portion of the stimulus is not the actual top (Jeerakathil \& Kirk, 1994).

Visual field comparisons have also been made by presenting horizontal stimuli to either the upper VF or the lower VF, but results from these studies have been mixed. The leftward bias has been shown to be stronger in both the upper VF (McCourt \& Garlinghouse, 2000; McCourt \& Jewell, 1999), and the lower VF (Barrett, Crosson, Crucian, \& Heilman, 2000; Thomas \& Elias, 2010). Due to the significant lack of prior research in this area, the goal of the current research was to further examine upper and lower VF differences in perceptual asymmetries as well as potential reasons for their occurrence.

Thomas and colleagues (2009) designed an exploratory computer-based route following task to examine whether spatial biases would emerge on a more "real-world" measure of perceptual asymmetries. Participants were encouraged to follow the route as quickly as possible and the number of collisions made with both the left walls and the right walls was recorded. Thomas et al. (2009) also explored the effect of upper and lower VF presentations on the number of collisions with each wall.

Thomas and Elias (2010) then examined whether the strength of the left bias on the greyscales task would be affected by VF or viewing distance and whether these factors would interact. The greyscales task was presented to either the upper or the lower VF, in both 
peripersonal and extrapersonal space. This allowed the potential relation between the lower VF and peripersonal space, and the upper VF and extrapersonal space (Previc, 1990) to be examined.

Following this, the relative position of the greyscale images was manipulated to look at how object- and space-based coordinates contribute to the left bias (Thomas \& Elias, in press). One image from each greyscale pair was shifted toward the left side or the right side of the screen to create either a partial or no overlap between the two images. Object-based biases were also examined using actual objects, with an obvious central axis, as the greyscale images do not have an easily identifiable midline. This allowed for a more ecologically valid comparison of object-and space-based biases.

Prior research looking at upper and lower VF differences had not used a consistent methodology, with various researchers employing different tasks and different presentation times (see Barrett et al., 2000; McCourt \& Garlinghouse, 2000; McCourt \& Jewell, 1999). The greyscales task was presented in the upper and lower VFs, using two different presentation times: prolonged (10 seconds) and brief $(150 \mathrm{msec})$. This was done to investigate the potential interaction of these two factors.

Although various explanations have been put forth to account for the left bias, none of these can adequately explain why upper and lower VF differences exist. It has been suggested that the upper VF is better processed by the ventral stream, whereas information in the lower VF is preferentially processed by the dorsal stream (Previc, 1990). It has been shown that when information is processed in peripersonal space, it leads to dorsal stream activation, whereas extrapersonal space processing is synonymous with ventral stream activation (Weiss et al., 2000). The possible contribution of dorsal or ventral stream processing to perceptual asymmetries was investigating by creating tasks that incorporated either motion or colour. 
It has been shown that the dorsal stream is consistent with the magnocellular pathway (Goodale \& Westwood, 2004; Livingstone \& Hubel, 1988), promotes temporal segregation, is advantaged in processing motion (Nieuwenhuis, Jepma, La Fors, \& Olivers, 2008) and is thought to be responsible for spatial judgments (Goodale \& Milner, 1992). In contrast, the ventral stream is an extension of the parvocellular pathway (Goodale \& Westwood, 2004; Livingstone \& Hubel, 1988), supports spatial segregation and is superior at processing colour (Nieuwenhuis et al., 2008). Tasks that target either dorsal (motion) or ventral (colour) stream functioning can isolate (for the most part) one visual stream, and should identify which preferentially contributes to the emergence of perceptual asymmetries.

\section{Rationale and Research Questions}

There has been a significant lack of prior research looking at upper and lower VF comparisons (only three studies: Barrett et al., 2000; McCourt \& Garlinghouse, 2000; McCourt \& Jewell, 1999). The purpose of the current research program was to examine upper and lower VF differences on the greyscales task, and on an exploratory computer-based route following task as a measure of real-world perceptual asymmetries (see Figure 1-1 for flow chart of studies). After finding that upper and lower VF differences occurred on both tasks, we examined factors that might influence upper and lower VF differences as well as possible explanations for why VF differences are seen in perceptual asymmetries. Several of these factors have not been considered previously and this therefore provides a significant addition to the literature. Further, reasons for why VF differences occur in perceptual asymmetries have not been addressed and the current findings shed light on this. The following questions were investigated:

1) Can lateralized bumping be observed on a computer-based route following task that exemplifies a more "real-world" measure of perceptual asymmetries? 
b. Do upper and lower VF differences occur on this task?

c. Does handedness influence the number and direction of collisions on this task? Does handedness influence upper and lower VF differences?

2) Do upper and lower VF differences occur on the greyscales task?

3) Do peripersonal and extrapersonal space differences occur on the greyscales task?

b. Is there an interaction between upper and lower VF differences and peripersonal and extrapersonal space processing on this task?

4) What are the contributions of object- and space-based coordinates to the left bias?

b. Do object- and space-based biases interact to influence the leftward bias?

c. What is the impact of the location of each greyscale rectangle relative to one another on the strength of the left bias?

5) Does the use of actual objects, with central axes, better clarify the contribution of object-based coordinates to the leftward bias?

b. Does the type of stimuli used lead one frame of reference to contribute more strongly to the left bias?

c. Does spatial location influence the type of coordinates that are relied upon?

6) Does length of presentation time (brief vs. prolonged) influence the strength of the left bias on the greyscales task?

b. Is there an interaction between length of presentation time and VF presentation (upper vs. lower) on this task?

c. Which areas of the VF do participants examine when performing the greyscales task and are their eye movements consistent with their responses? 
7) Can the leftward bias be diminished by using tasks that incorporate motion or colour to target either dorsal or ventral stream processing?

b. Does length of presentation time (brief vs. prolonged) influence the strength of the left bias on the motion task?

c. Does length of presentation time (brief vs. prolonged) influence the strength of the left bias on an isoluminant red-green colour task?

d. Does the colour red create a potential confound in visuospatial attention research? Can this be eliminated by using isoluminant blue-yellow stimuli instead?

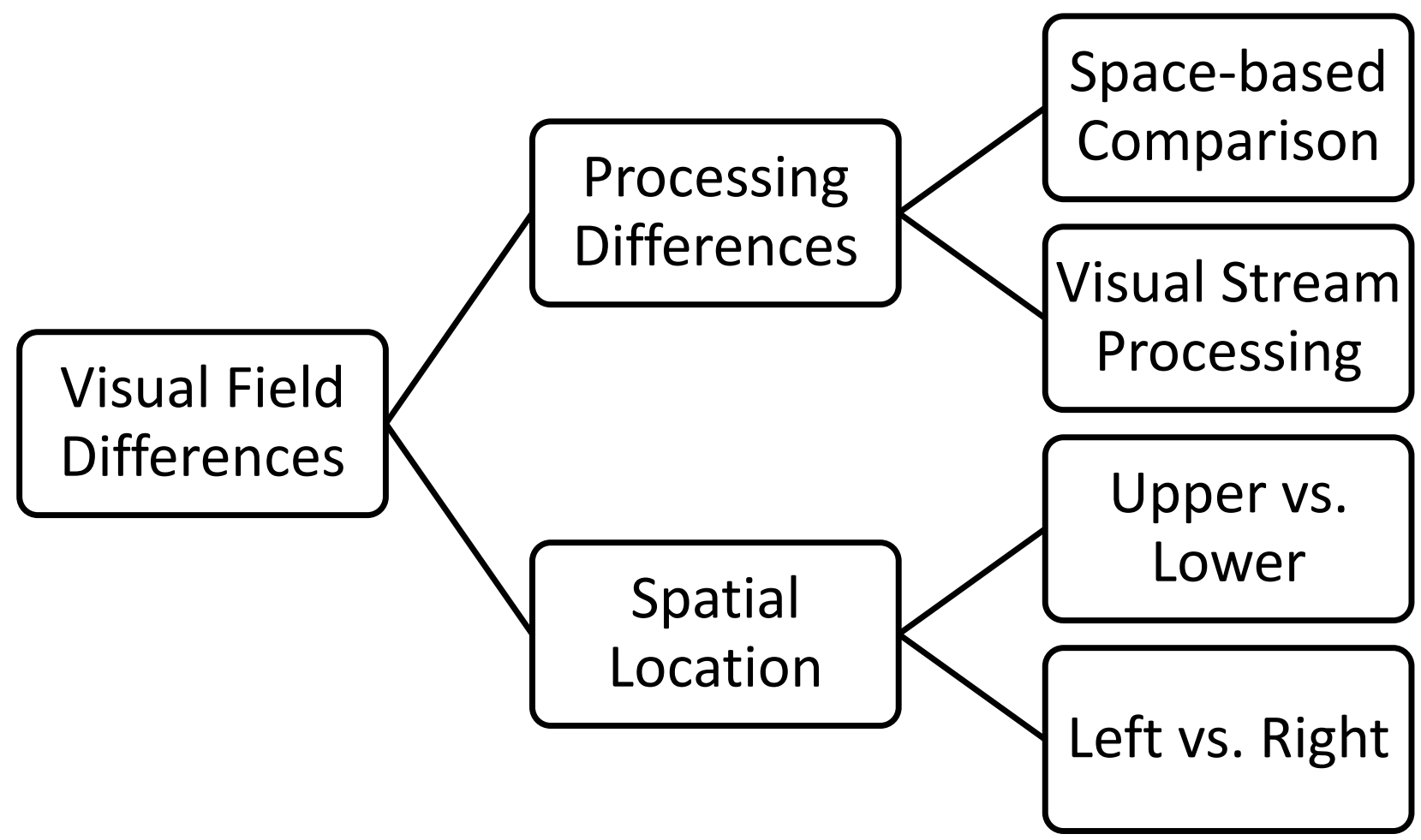

Figure 1-1. Description of the different ways in which visual field differences were examined in the current research program. The first stream examined processing differences by comparing space-based differences in peripersonal vs. extrapersonal space. The second stream examined spatial location by comparing upper and lower visual field differences directly as well as their relation to the left and right visual field differences. 


\section{CHAPTER 2 \\ DIRECTIONAL COLLISIONS DURING A ROUTE FOLLOWING TASK}

\section{Introduction}

Hemispatial neglect patients with good walking ability tend to collide with objects located on the left side of their body more frequently than with objects located on the right side (Grossi, Lepore, Napolitano, \& Trojano, 2001; Huitema et al., 2006; Robertson, Tegnér, Goodrich, \& Wilson, 1994). This has been referred to as lateralized bumping and it has been observed that normal individuals experience a similar (although nonsignificant), but opposite lateral bias (Turnbull \& McGeorge, 1998). Turnbull and McGeorge (1998) investigated selfreported lateralized bumping and found that individuals report colliding into objects on the right

side of their body more often. They also found that individuals who reported bumping their right side showed a significant leftward bias on line bisection (i.e., bisect to the left of centre), illustrating a potential relationship between lateralized bumping and another measure of perceptual asymmetry. However, as self-report measures are susceptible to false memory (Hyman \& Loftus, 1998; Winograd, Peluso, \& Glover, 1998) they may not reflect real-world behaviour, making an objective behavioural measure desirable.

Nicholls and colleagues (2007) further investigated lateralized bumping by constructing an adjustable doorway for participants to walk through. Participants were asked to shoot a toy gun at a target while walking through the doorway using either their left hand or their right hand, or both hands. When targeting with their left hand, participants were more likely to collide with the right side of the doorway, while the opposite was true when using the right hand. In the bimanual condition, thought to reflect bilateral activation, participants experienced more collisions with the right side. Further, across conditions, more right side collisions occurred, providing behavioural evidence of lateralized bumping. 
A recent study investigated perceptual asymmetries when individuals are asked to make distance judgments. Krupp et al. (2010) examined perceptual asymmetries for distance judgments by presenting a pair of three-dimensional rectangle boxes in the centre of the screen. Each box had a dark square representing the front. Both boxes were identical but mirror-reversed so that one box was facing slightly left and the other slightly right. Participants were asked to judge which of the pair appeared to be closer to them. Krupp and colleagues (2010) found that when the box was facing left it was judged to be closer than the rightward facing box, despite the boxes being equidistant. This was indicative of a leftward bias (i.e., chosen as being closer despite a lack of difference in distance) and the authors concluded that this bias in distance judgments may contribute to the phenomenon of lateralized bumping.

Perceptual asymmetries have been examined extensively using the greyscales task (Mattingley et al., 2004; Nicholls et al., 1999; Okubo \& Nicholls, 2006). In the greyscales task, participants are presented with a pair of equiluminant, mirror-reversed rectangles that are bright on one side and dark on the other. Images are presented parallel to one another and participants are asked to select which image appears darker. Participants tend to select the image that is dark on the left side as being darker overall, despite the images being identical (Mattingley et al., 2004), referred to as pseudoneglect (Bowers \& Heilman, 1980; Jewell \& McCourt, 2000). When performed by hemispatial neglect patients who have suffered right parietal damage (Brain, 1941; Vallar \& Perani, 1986), the greyscales task shows a similar but opposing rightward bias (Mattingley et al., 2004). The greyscales task exhibits a bias similar to that observed on line bisection tasks (Mattingley, Pierson, Bradshaw, Phillips, \& Bradshaw, 1993); however the greyscales are a newer task that have demonstrated both large and reliable effects (i.e., Nicholls 
et al., 1999). Further, using the greyscales, Nicholls and Roberts (2002) demonstrated that the leftward bias likely results from an attentional bias and not a pre-motor or scanning bias.

Horizontal biases have also been examined in the upper and lower VFs; however research in this area has been inconclusive. Examinations using a landmark task where participants are presented with a pre-transected line and asked to indicate whether the transector is to the left or right of centre have suggested the presence of a stronger leftward bias in the upper VF (McCourt \& Garlinghouse, 2000; McCourt \& Jewell, 1999). However, Barrett et al. (2000) found a stronger leftward bias in the lower VF using a line bisection task. This indicates a need for further research to understand the nature of VF differences on perceptual asymmetries.

Handedness influences performance on various cognitive and perceptual tasks (Jewell \& McCourt, 2000). Several reports have included hand use effects on line bisection by having participants bisect with both hands; however few studies have investigated the effect of handedness. Left-handed participants have been found to bisect farther to the left of centre than right-handers (Luh, 1995; Scarisbrick, Tweedy, \& Kuslansky, 1987); however bisections for both handedness groups are typically leftward (Luh, 1995; Sampaio \& Chokron, 1992). Although this illustrates that perceptual asymmetries are influenced by handedness, the nature of this effect is not clearly understood.

The current study further examined the factors listed above, with the goal being to determine whether more right side collisions would occur on a computer-based route following task. An exploratory route following task was used to assess directional differences in the number of collisions participants experienced. Further, upper and lower VF differences during the route following task were explored by including three presentation conditions: central as well as upper and lower VF conditions. 
Neurologically normal individuals have shown a leftward bias on the greyscales task (implying greater attention to the left) and lateralized bumping measures demonstrate a right side collision bias, also suggesting an attentional bias. Therefore, performance on these two tasks should be correlated. Further, individuals show a stronger leftward bias in the upper VF on the landmark task, which is also an attentional measure. In contrast, performance on line bisection tasks (i.e., Barrett et al., 2000) suggests that the leftward attentional bias (leading to right side collisions) may be moderated by leftward motor biases (causing left side collisions) in the lower VF. These results would predict that performance on the current task would lead to more right side collisions in the upper VF, where attentional biases would dominate. In the lower VF, the number of right side collisions should be reduced as leftward motor biases oppose or potentially surpass the effects of the attentional bias. In addition, performance in the upper VF would be predicted to be correlated with performance on the greyscales task, whereas performance in the lower VF would only correlate weakly.

\section{Method}

\section{Participants}

A total of 52 University of Saskatchewan students (17 male; mean age $=20.94, S D=4.47)$ were tested. Three participants were excluded due to technical errors in recording their data. Four participants were classified as left-handed (two male) based on scores from the Waterloo Handedness Questionnaire (see Appendix A; Elias, Bryden, \& Bulman-Fleming, 1998). Students received course credit in exchange for their participation. This study was conducted with the ethical approval of the Behavioural Research Ethics Board at the University of Saskatchewan and has therefore been performed in accordance with the ethical standards laid down in the 1964 Declaration of Helsinki. 


\section{Materials}

Waterloo Handedness Questionnaire-Revised. The questionnaire consisted of 15 questions assessing hand preference on various tasks and 3 questions related to potential reasons for hand preference (see Appendix A). Participants responded on a five-point likert-type response scale consisting of right hand always (score of +2$)$, right hand usually $(+1)$, equally often (0), left hand usually (-1), and left hand always (-2) in terms of preference. These values were then summed to create an overall handedness score (range +30 to -30 ), where positive scores indicate an overall right preference and negative scores an overall left preference. Participants were classified as either left-handed or right-handed depending on whether their scores were positive or negative. This questionnaire was taken from Elias et al. (1998).

Computer-based Route Following Task. Stimuli were administered on an IBM clone computer (PIV 2.4 GHz) interfaced with a 19" SVGA CRT monitor running at 1024 x 768 resolution. A computer-based program used bitmaps (128 x 128 pixels) to generate three possible pathways for participants to follow (see Appendices B and C). All 3 pathways consisted of 26 left turns and 26 right turns. There were no wrong turns or dead ends along the pathway, removing the need for path selection during the task. Three presentations were used: upper VF, lower VF, or central view. The size of the pathway was held consistent in each condition as the view of the entire screen was shifted either above or below centre. During the upper and lower VF presentations the opposing field was blacked out to ensure that participants were directing their attention to the desired field. The order of presentation was counterbalanced among participants and each presentation followed a different pathway (also counterbalanced) to avoid practice effects. 
Participants were seated directly in front of the computer in the same location and neither the computer nor the screen was moved between participants. All participants were told to use the forward and side arrow keys on the keyboard to move along the pathway. All participants performed the task with their right hand as the keypad was located on the right side of the keyboard and it would not be desirable for participants to use their left hand in the right side of space. Although left-handed participants did not complete the task with their preferred hand, prior research indicates that hand use may also influence performance (i.e., Jewell \& McCourt, 2000) and therefore this factor was controlled. Further there were no reaction times differences between handedness groups [repeated-measures analysis of variance (ANOVA): $F(2,94)=1.102$, $p=.337$ ] illustrating that left-handed participants (upper: $M=181.78 \mathrm{~s}, S D=103.78$; central: $M=153.92, S D=75.47$; lower: $M=147.31, S D=59.84)$ were able to complete the task in a similar fashion to the right-handed participants (upper: $M=161.32, S D=68.45$; central: $M=163.43$, $S D=63.86$; lower: $M=157.69, S D=71.80)$.

Participants were naïve to the purpose of the task but were told to complete the route as quickly as possible, and that there were no wrong turns or dead-ends. The pathway was not visible to participants and they were not provided with a map or with directions. Time taken to complete the task, as well as the number of collisions with the right walls and left walls, was automatically recorded. Participants were given an unlimited amount of time [mean completion time for upper $(M=162.97 \mathrm{~s}, S D=70.71)$, central $(M=162.65 \mathrm{~s}, S D=64.04)$, and lower $(M=156.85 \mathrm{~s}$, $S D=70.41)]$. Bias scores were created for each condition by subtracting left side collisions from right side collisions and dividing by the total number of collisions. An overall bias score was also created by summing the total number of left side collisions and right side collisions across presentation conditions. 
Greyscales Task. E-prime (Psychology Software Tools, Inc., Pittsburgh, USA; www.pstnet.com/eprime) was used to administer a series of 36 greyscales, of varying lengths, on the same computer described above. Two mirror-reversed images were presented in the central VF, one directly above the other, one of the images being dark on the left side and the other dark on the right (see Appendix D). Participants were asked to select whether the image on the top or the bottom appeared darker, with the instructions emphasizing the forced-choice nature of the task. Key press responses, as well as response times, were recorded. A response bias score was calculated by subtracting the number of leftward responses from the number of rightward responses, with a negative score indicating a leftward bias (Nicholls et al., 1999). This task has been used extensively in examinations of perceptual asymmetries as the key press required by this task minimizes motor involvement in comparison to that required in line bisection tasks (i.e., McCourt \& Olafson, 1997).

Lateralized Bumping Questionnaire. This questionnaire (see Appendix E) was given as a measure of lateralized bumping, which could be correlated with performance on the route following task. Participants were asked if they had ever bumped themselves against an object while concentrating on something else. They were to then describe the specifics of the incident, including the part and side of the body that they bumped. All participants were instructed to answer the first question and those who responded no to this question were instructed to return the questionnaire to the experimenter. Additionally, those participants who could not recall a specific incident were instructed to indicate so on the questionnaire and return it to the experimenter. As only preliminary research has been conducted in this area (see Turnbull \& McGeorge, 1998), psychometrics are not available for this questionnaire. 


\section{Procedure}

Following informed consent (see Appendix F), participants completed the Waterloo Handedness Questionnaire-Revised (see Appendix A; Elias et al., 1998). The questionnaire also contained additional demographic questions, such as sex, age, visual or hearing impairments and familial sinistrality. Following this, participants completed three route following tasks, followed by the greyscales task, and lastly the lateralized bumping questionnaire. All participants were debriefed following participation (see Appendix G).

\section{Results}

A $3 \times 2 \times 2$ repeated measures ANOVA with within-subjects factor presentation condition (upper field, central, lower field) and between-subjects factors handedness (righthanded, left-handed) and sex (male, female) was computed to examine directional bias for collisions on the route following task; however none of the effects related to sex reached significance (all $F<1$, all $p>.433$ ). The dependent measure was the bias score for each presentation condition. There was a significant main effect of presentation condition, $F(2,94)=7.779, p=.001$. Paired-samples t-tests indicated that there was a significant difference between the upper and lower VF presentations $t(48)=2.913, p=.005$, with the lower presentation demonstrating more left side collisions $(M=-.135, S D=.381)$ and the upper presentation demonstrating more right side collisions $(M=.075, S D=.274$; both significantly different from zero, $t(48)=-2.475, p=.009$ and $t(48)=1.912, p=.031$, respectively). The lower VF presentation was also significantly different from the central presentation $(t(48)=2.525, p=.015)$, which did not exhibit a directional bias $(t(48)=-1.006, p=.160, M=.051, S D=.356)$.

This main effect was qualified by a significant handedness by presentation condition interaction, $F(2,94)=3.794, p=.026$. Post hoc comparisons indicated that handedness did not 
influence performance in either the upper or the lower field conditions; however left-handed individuals had significantly more right side collisions in the central condition $t(47)=-2.237$, $p=.030$ (See Figure 2-1).

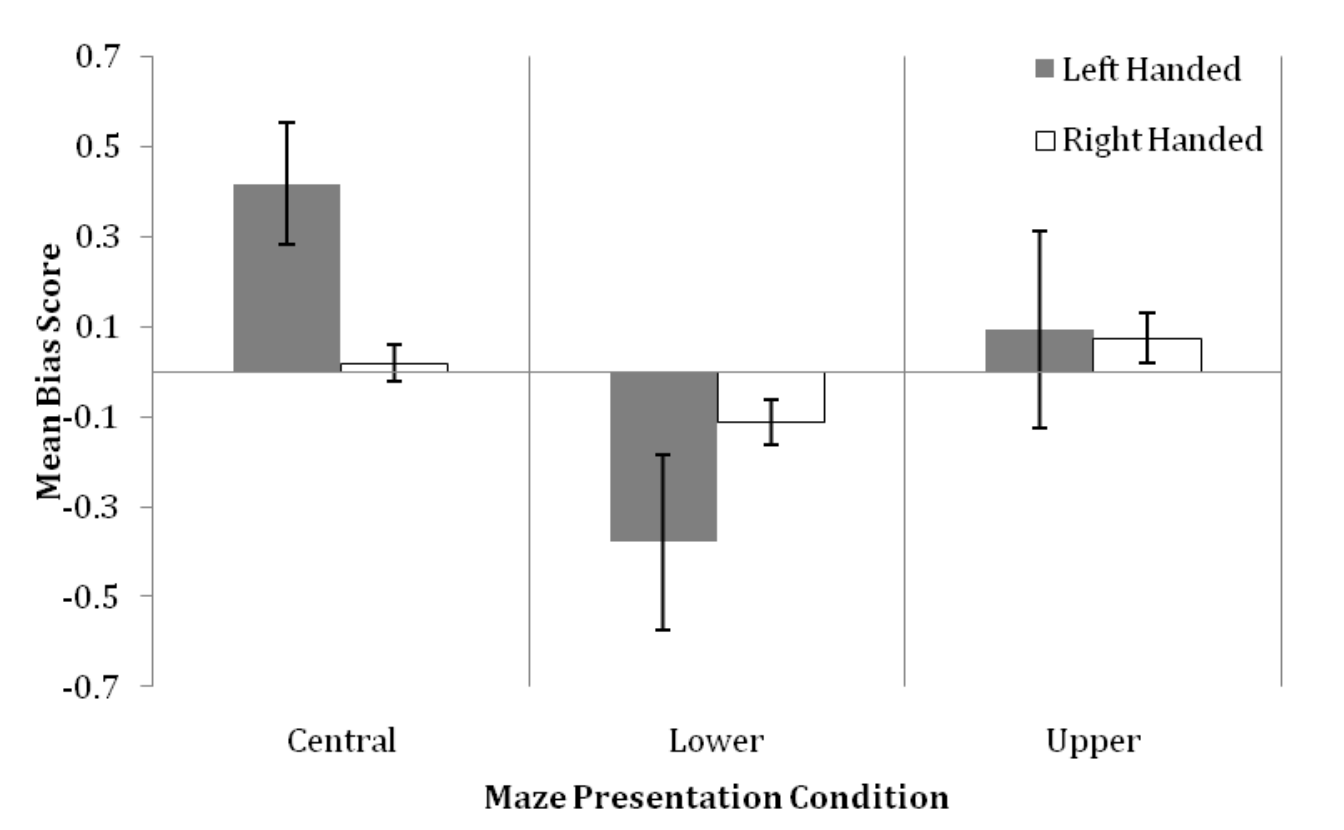

Figure 2-1. Handedness by presentation condition interaction. Values represent mean bias scores obtained by subtracting the number of leftward collisions from the number of rightward collisions, with positive values indicating a rightward bias and negative values a left side bias. Error bars represent the standard errors of the mean.

The overall number of left side collisions and right side collisions (collapsed across conditions) was also examined; however no significant overall bias emerged, $t(48)=.586, p=.561$, $M=.014, S D=.164$. The total number of collisions in each condition was compared to assess whether more collisions were recorded in any one presentation condition. A repeated-measures ANOVA with presentation condition (upper, central, lower) as the within-subjects factor revealed a main effect of presentation condition $F(2,96)=4.625, p=.012$. Paired-samples t-tests revealed that there were more collisions overall in the upper VF condition $(M=36.53, S D=29.36)$ when compared with both the central condition $t(48)=2.314, p=.025(M=29.24, S D=22.66)$ and 
the lower VF condition $t(48)=2.468, p=.017(M=28.57, S D=24.68)$. The central and lower VF conditions did not differ from one another.

A one-sample t-test was computed for bias scores on the greyscales task and a significant leftward bias emerged, $t(26)=-3.215, p=.003, M=-10.37, S D=16.76$. Although the self-report measure indicated slightly more right side bumps, this difference was not significant, $t(47)=1.159, p=.252$. Pearson-Product Moment correlations were performed to examine potential relationships among the three conditions on the route following task as well as the greyscales task and the self-report measure; however all failed to reach significance (all $p$ values>.05; See Figure 2-2).

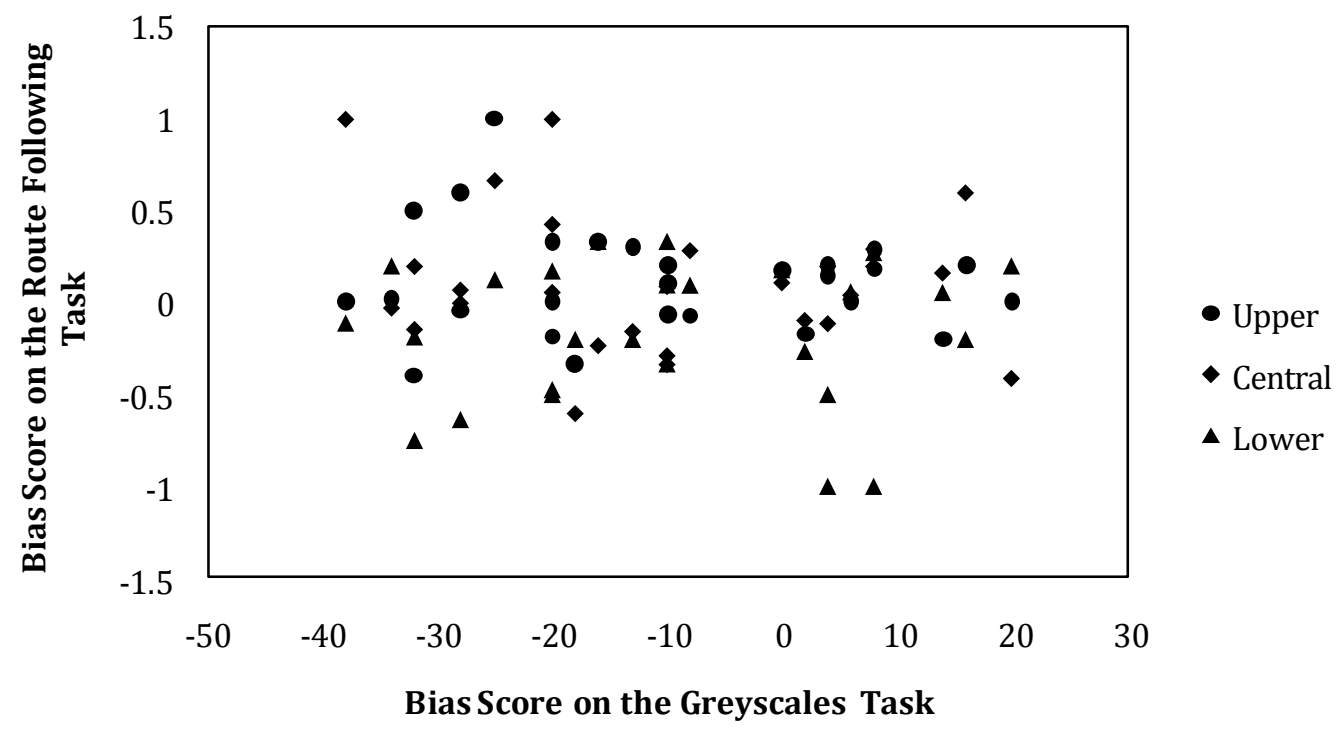

Figure 2-2. Scatterplot illustrating the lack of relationship between the greyscales task and each of the conditions of the route following task.

\section{Discussion}

A computer-based route following task was used to compare the number of collisions made with the left walls and the right walls. In the central condition, participants did not demonstrate a bias, which was contrary to the expectation of more right side collisions in this condition. When the task was performed in either the upper or the lower VF, significant biases 
were observed. Participants collided with the walls on the right side more often in the upper VF condition; however when performing the task in the lower VF, collisions occurred more often with the left side.

The finding of more right side collisions in the upper VF is consistent with the findings of McCourt and colleagues $(1999,2000)$ regarding upper and lower VF biases. The incidence of more right side collisions could reflect either a slight neglect of the right side or an overestimation of the left side. McCourt et al. (1999; 2000) observed stronger leftward biases in the upper VF using a landmark task, which would be consistent with the occurrence of more right side collisions in the upper VF. It was suggested that in the lower VF, the leftward attentional bias may be moderated by leftward motor biases that would decrease or possibly even reverse the right side collision bias. Therefore, the left side collision bias observed in the lower VF was also in line with hypotheses.

Handedness was found to influence performance on the route following task in the current study; however as the number of left-handed participants was very small, no firm conclusions can be made. In the central condition, left-handed participants experienced more right side collisions than right-handed participants. As prior research examining handedness in relation to perceptual asymmetries has shown a stronger leftward bias (i.e., Luh, 1995; Sampaio \& Chokron, 1992; Scarisbrick et al., 1987), it follows that left-handed individuals would experience significantly more right side collisions. Nicholls and colleagues' (2007) examination of lateralized bumping did not find an effect of handedness; however as research in this area is preliminary, it remains possible that laterality influences performance. These findings suggest that handedness effects should be considered in future investigations of directional collisions to better illustrate whether laterality contributes significantly to performance. 
The finding of a lack of correlation between performance on the route following task and the greyscales task was not consistent with expectations. As research linking performance on perceptual asymmetries tasks with lateralized bumping has been inconclusive, it is possible that performance on the current task is not related to perceptual asymmetries. Future research should examine performance on the greyscales task in the upper and lower VFs as performance on this measure should be similar to that observed on the landmark task (McCourt \& Garlinghouse, 2000; McCourt \& Jewell, 1999), also an attentional measure. It appears that vertical biases differ for attentional and motor biases whereby stimuli that are higher in the VF demonstrate a stronger attentional bias; however stimuli that are lower in the VF show a stronger motor bias.

Preliminary investigations of lateralized bumping have suggested that right side collisions occur more often than left side collisions (Nicholls et al., 2007; Turnbull \& McGeorge, 1998). The current study further suggests the existence of a directional difference in the number of collisions that individuals experience; however it appears that this effect is further mediated by the vertical VF in which the task is carried out. The exploratory route following task used in the current study also appears to be a useful measure that merits inclusion in future studies examining this phenomenon. Other factors that should be considered include further examination of handedness differences by including equal numbers of left-handed and right-handed participants as well as a specific comparison of performance on the route following task in near and far space. Further, handedness comparisons related to hand use should also be considered in future research. This may be able to shed light on the nature of upper and lower VF differences in directional collisions and potentially also perceptual asymmetries. 


\section{CHAPTER 3 \\ DO PERCEPTUAL ASYMMETRIES DIFFER IN PERIPERSONAL AND \\ EXTRAPERSONAL SPACE?}

\section{Introduction}

It has been suggested that differences in the upper and lower VF might relate to extrapersonal and peripersonal space differences, respectively (Previc, 1990). Previc (1990) suggests that stimuli that are located in the lower VF are more likely to be found in peripersonal space, whereas those located in the upper VF are more likely to be located at a distance, in

extrapersonal space. Further, it is suggested that when an object is brought into peripersonal space, it will likely also be brought into the lower VF (Previc, 1990). These differences are additionally thought to be associated with dorsal and ventral visual stream processing differences.

Weiss et al. (2000) demonstrated that when acting in peripersonal space there is greater neural activity in the dorsal stream; however ventral stream activity is greater during extrapersonal space processing. A space-based dissociation has also been shown in clinical hemispatial neglect as it can occur solely in peripersonal space (Halligan \& Marshall, 1991), or alternatively, can be stronger in extrapersonal space (i.e., Keller, Schindler, Kerkhoff, von Rosen, \& Golz, 2005), suggesting that distance-related differences might occur in pseudoneglect (Bowers \& Heilman, 1980) as well.

Various tasks have been used to examine pseudoneglect such as line bisection and landmark tasks (i.e., Luh, 1995; McCourt \& Jewell, 1999). On these tasks typically individuals exhibit a lateral bias where they are more likely to bisect lines to the left of centre or to indicate that a transection mark is located to the left of centre (landmark task). It has been suggested that this reliably observed phenomenon is similar to clinical hemispatial neglect and therefore has 
been referred to as pseudoneglect (Bowers \& Heilman, 1980). Examinations of pseudoneglect in peripersonal and extrapersonal space using line bisection and landmark tasks have typically found that the leftward bias is stronger in peripersonal space than extrapersonal space (Bjoertomt et al., 2002; Garza, Eslinger, \& Barrett, 2008), with one study identifying a progressive bias that became rightward in extrapersonal space (Varnava, McCarthy, \& Beaumont, 2002).

Line bisection tasks are typically performed in extrapersonal space through the use of a laser pointer. Interestingly, Longo and Lourenco (2006) found that although the leftward bias shifted rightward when a laser pointer was used during bisection, the use of a stick led to a consistent leftward bias, regardless of distance. They concluded that the use of a stick allowed extrapersonal space to be remapped as peripersonal space, allowing the leftward bias to persist. When using a laser pointer this remapping did not occur and areas typically activated by peripersonal space (i.e., intraparietal sulcus in the right hemisphere; Bjoertomt et al., 2002) were no longer activated, leading to a rightward shift in bias (Longo \& Lourenco, 2006).

Alternatively, Varnava et al. (2002) suggest that each cerebral hemisphere has a unique spatial coordinate system and that as one system is used more or less prominently there is a gradual shift in coordinate system, leading to opposing biases in peripersonal and extrapersonal space. They propose that the progressive nature in which the bias becomes rightward points to a gradual shift as opposed to an abrupt switch from the left side to the right. This suggestion is consistent with the findings of Longo and Lourenco (2006). However, Wilkinson and Halligan (2003) did not find distance-based differences when the visual angle of the image was held constant, highlighting the importance of controlling this factor.

Initial research examining upper and lower VF differences on line bisection and landmark tasks has been conducted; however results of these studies have been inconsistent. McCourt and 
Jewell (1999) and McCourt and Garlinghouse (2000) compared performance on a landmark-type task in the upper and lower VFs using tachistoscopic presentation $(150 \mathrm{msec})$. Lines were presented at $3.6^{\circ}$ and $5.8^{\circ}$ visual angle, respectively. Participants were asked whether a transector was located to the left or right of centre. In both instances, a stronger leftward bias was identified in the upper VF compared with the lower VF.

A third investigation by Barrett et al. (2000) used a manual line bisection task (unlimited completion time) presented at $40.5 \mathrm{~cm}$ either above or below the midsagittal plane. Barrett and colleagues (2000) identified a stronger leftward bias in lower body space as opposed to upper body space. It is difficult to draw any strong conclusions relating to these findings as all three studies were methodologically different. It is clear that the results of these studies are not consistent with one another.

In order to better understand how perceptual asymmetries are affected by viewing distance, the greyscales task was presented in both peripersonal and extrapersonal space. Further, to increase understanding of how VF differences and space-based differences relate, this task was presented to both the upper and the lower VF. Previous findings have been contradictory, suggesting that both space-based and VF differences should be examined in one manipulation to better understand their relation as well as how each individually influences perceptual asymmetries.

The greyscales task has been used to examine perceptual asymmetries (Nicholls et al., 1999). This task involves making a judgement between two equiluminant mirror-reversed rectangles going from black on one side to white on the other, one rectangle being dark on the left and the other dark on the right. When asked to select which image appears to be darker (or brighter) overall, most often individuals will choose the rectangle with the salient feature on the 
left side, despite the images being equiluminant (Nicholls et al., 1999). Kinsbourne (1970) hypothesized that due to right hemisphere involvement in attention, attentional vectors are preferentially directed to the left side of space. Therefore, the leftward bias observed on this task is thought to reflect increased attention being directed to the left side, and leading to a left side spatial bias. Similar tasks have been used to examine judgements of size, numerosity (Nicholls et al., 1999), and distance (Krupp et al., 2010), all of which also demonstrate leftward biases.

There have been a number of suggested explanations, including scanning and reading habits (Chokron, Bartolomeo, Perenin, Helft, \& Imbert, 1998; Manning, Halligan, \& Marshall, 1990), motor biases, and intentional or pre-motor biases (Brodie \& Pettigrew, 1996; Heilman \& Valenstein, 1979). Nicholls and Roberts (2002) carried out a series of experiments demonstrating that these explanations do not fully account for observed leftward biases. Individuals with opposite reading patterns demonstrated leftward biases and bimanual responding led to leftward biases as well, illustrating that these explanations are incomplete.

Attentional or perceptual biases currently appear to be the most plausible explanation for perceptual asymmetries. The leftward bias also occurs during tachistoscopic presentation (McCourt \& Jewell, 1999), supporting the idea that left biases are attentional in nature. A final explanation, although preliminary, also appears promising. It has been shown that birds demonstrate a leftward bias on perceptual asymmetries tasks, which suggests the possibility of an evolutionary basis for the leftward bias (Diekamp, Regolin, Güntürkün, \& Vallortigara, 2005; Regolin, 2006). However, this explanation is quite preliminary and admittedly difficult to examine in a human population.

Prior research indicates a need to evaluate space-based differences in perceptual asymmetries by using a paradigm that is easily performed in both peripersonal and extrapersonal 
space. The current study examined both peripersonal and extrapersonal space differences and VF differences using the greyscales task. Although a stronger leftward bias has been previously observed in extrapersonal space, it was hypothesized that when the visual angle of the stimuli was kept constant, (i.e., Dellatolas, Vanluchene, \& Coutin, 1996; Wilkinson \& Halligan, 2003), there would not be any distance-based differences in perceptual asymmetries. Controlling the visual angle of the stimulus would ensure that the relative size of the stimulus remained constant and did not create a confound. We also investigated VF differences by presenting greyscale pairs to either the upper or the lower VF. As McCourt and Garlinghouse (2000) and McCourt and Jewell (1999) found a stronger leftward bias in the upper VF using an attention-based landmark task, it was expected that the leftward bias would be stronger in the upper compared with the lower VF. It has been suggested that upper and lower VF differences may correspond with extrapersonal and peripersonal space; however as distance-based differences were not expected, this interaction was not expected to be observed.

\section{Method}

\section{Participants}

Thirty-nine right-handed undergraduate Psychology students (10 males; mean age=19.72, $S D=3.74$ ) at the University of Saskatchewan participated in the current study. Students received course credit in exchange for participation. Based upon self-report (see Appendix A; Elias et al., 1998), all participants had normal or corrected-to-normal vision. This study was conducted with the ethical approval of the Behavioural Research Ethics Board at the University of Saskatchewan and has therefore been performed in accordance with the ethical standards laid down in the 1964 Declaration of Helsinki. 


\section{Materials}

Greyscales Task. E-prime (Psychology Software Tools, Inc., Pittsburgh, USA; www.pstnet.com/eprime) was used to administer a series of 320 greyscales, of 5 different lengths: 110, 132, 154, 176 and $198 \mathrm{~mm}$. Eighty of the greyscales were two mirror-reversed images where one appeared directly above the other, with one of the images being dark on the left side, whereas the other was dark on the right. The remaining 240 greyscales were not mirrorreversed in that one greyscale was 250,500 , or 1000 pixels darker than the other was. Greyscales were at $9.2^{\circ}$ visual angle, either above or below the centre of the screen with 160 trials in each VF condition (upper, lower; see Appendix H). As it is important to control visual angle when looking at distance-based differences (i.e., Dellatolas et al., 1996), visual angle was maintained across conditions. This task can be easily performed in extrapersonal space through the use of a data projector.

There were 2 viewing distances: peripersonal space and extrapersonal space, each with 160 trials. Stimuli in the peripersonal space condition were administered on an IBM clone computer (PIV 2.6 GHz) interfaced with a 19” LCD monitor running at 1024 x 768 resolution. The maximum resolution of the monitor was 1400 x 1050 and the refresh rate was $8 \mathrm{~ms}$. Participants used a chin rest in this condition to minimize head movement and distance to the screen was $71.1 \mathrm{~cm}$. In the extrapersonal space condition, an IBM laptop computer interfaced with a Sony VPL-ES3 3LCD projector was used to project stimuli onto a screen at a distance of $289.6 \mathrm{~cm}$ from the participant. As it was not possible to use a chin rest in the extrapersonal space condition, participants were asked to remain as still as possible for the duration of the task.

Participants were asked to select whether the image on the top or the bottom of the pair appeared darker. A central fixation was presented prior to each trial and participants were free to 
scan the images prior to responding. The greyscales task does not require significant motor involvement for responses, which were made with the right hand using the " $t$ " and " $b$ " keys, where " $t$ " represents the top image being chosen, and " $b$ " represents the bottom image being chosen. Responses, as well as response times, were recorded. Participants were given a maximum of five seconds to respond and no one exceeded this maximum. A response bias score was calculated by subtracting the number of leftward responses from the number of rightward responses, with a negative score indicating a leftward bias (Nicholls et al., 1999).

\section{Procedure}

Following informed consent (see Appendix I), participants completed a demographic questionnaire addressing items such as sex, age, visual or hearing impairments, and handedness and footedness (see Appendix A; Elias et al., 1998). Following completion of the questionnaire, participants completed the greyscales task twice, once in peripersonal space and once in extrapersonal space, with presentation order being counterbalanced. All participants were debriefed following participation (see Appendix J).

\section{Results}

A $2 \times 2 \times 5$ repeated-measures ANOVA was computed with within-subjects independent variables of VF (upper, lower), viewing distance (peripersonal, extrapersonal) and length of the greyscale $(110,132,154,176,198)$, and the dependent variable being bias score on the greyscales task. There was no main effect of viewing distance, $F(1,38)=.023, p=.879$, demonstrating that performance did not differ between peripersonal and extrapersonal space. There was no significant main effect of length, $F(4,152)=1.758, p=.140$. The interaction of viewing distance and VF also failed to reach significance, $F(1,38)=.878, p=.355$. There was an 
overall main effect of $\mathrm{VF}, F(1,38)=28.288, p<.001$, indicating a stronger leftward bias in the lower VF than the upper one $(p<.001$; see Figure 3-1).

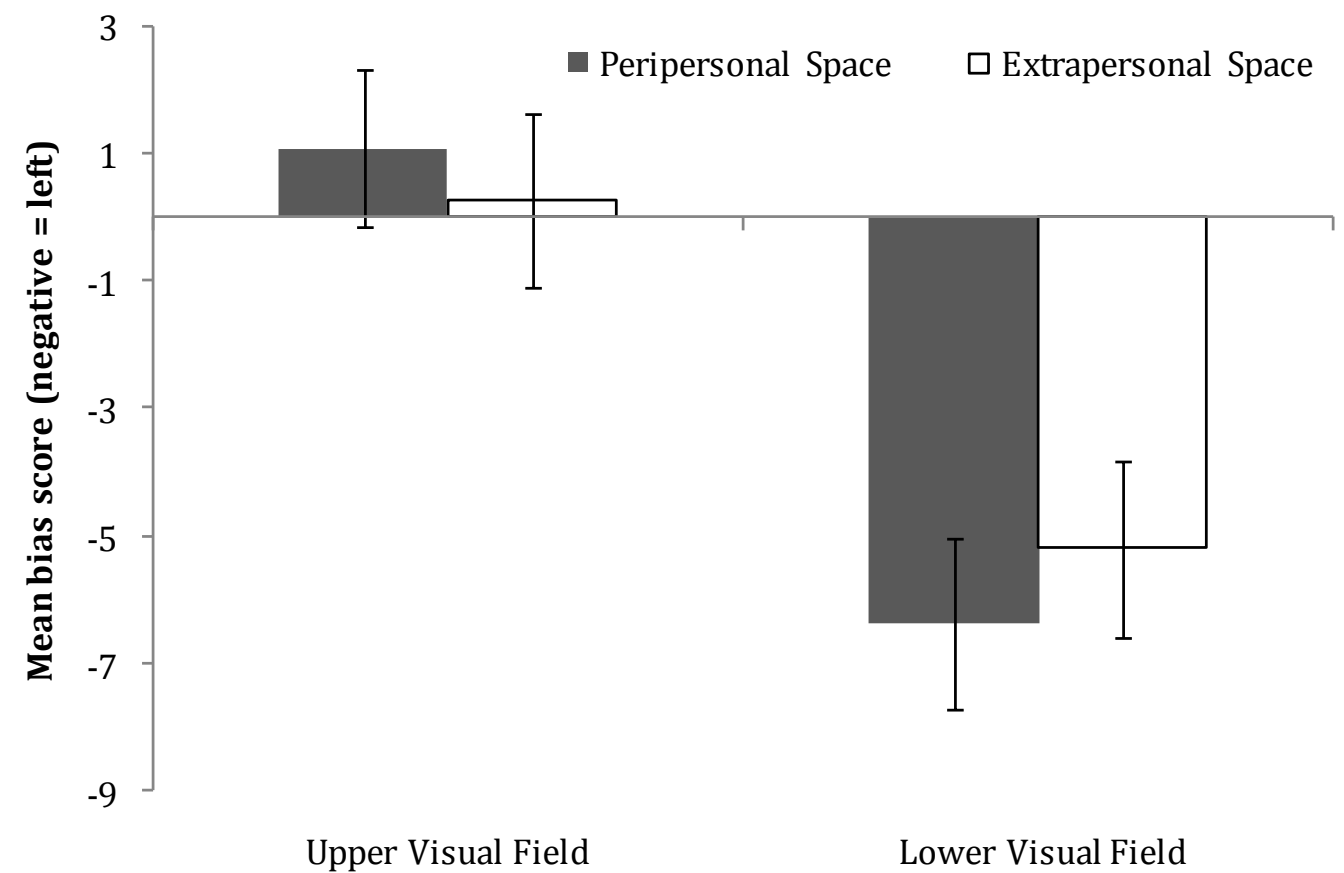

Figure 3-1. Interaction of visual field and viewing distance. Error bars represent standard errors of the mean.

Significant interactions between length and VF $(F(4,152)=9.929, p<.001)$ and between length and viewing distance were also observed $(F(4,152)=2.689, p=.033)$. For the length by VF interaction, post hoc paired-samples $t$-tests showed that a stronger left bias occurred in the lower VF for the 720 pixel length than for the $400(p=.015)$ and $640(p=.008)$ pixel lengths. In the upper VF, post hoc paired-samples $t$-tests showed that a stronger left bias occurred for the 400 $(p<.001), 480(p<.001)$, and $640(p<.001)$ pixel lengths when compared with the 720 pixel length. There was also a stronger left bias for the $480(p=.004)$ and $640(p<.001)$ pixel lengths compared with the 560 pixel length. The 640 pixel length also had a stronger left bias than the 400 pixel length $(p=.034)$.

For the length by viewing distance interaction, post hoc paired-samples $t$-tests indicated that in extrapersonal space, the greyscale that was 640 pixels in length had a significantly 
stronger leftward bias than all other lengths (all $p$ 's <.014). No other significant differences were seen for the length by viewing distance interaction.

Table 3-1. Accuracy scores based on luminance differences in Study 2

\begin{tabular}{|c|c|c|c|}
\hline Luminance Condition & Viewing Distance & Visual Field & Rate of left bias \\
\hline \multirow[t]{4}{*}{ Equiluminant } & \multirow[t]{2}{*}{ Peripersonal } & Upper & $61 \%$ \\
\hline & & Lower & $62 \%$ \\
\hline & \multirow[t]{2}{*}{ Extrapersonal } & Upper & $61 \%$ \\
\hline & & Lower & $66 \%$ \\
\hline \multirow[t]{4}{*}{250 pixel difference } & \multirow[t]{2}{*}{ Peripersonal } & Upper & $63 \%$ \\
\hline & & Lower & $64 \%$ \\
\hline & \multirow[t]{2}{*}{ Extrapersonal } & Upper & $65 \%$ \\
\hline & & Lower & $61 \%$ \\
\hline \multirow[t]{4}{*}{500 pixel difference } & \multirow[t]{2}{*}{ Peripersonal } & Upper & $70 \%$ \\
\hline & & Lower & $72 \%$ \\
\hline & \multirow[t]{2}{*}{ Extrapersonal } & Upper & $75 \%$ \\
\hline & & Lower & $71 \%$ \\
\hline \multirow[t]{4}{*}{1000 pixel difference } & \multirow[t]{2}{*}{ Peripersonal } & Upper & $78 \%$ \\
\hline & & Lower & $79 \%$ \\
\hline & \multirow[t]{2}{*}{ Extrapersonal } & Upper & $83 \%$ \\
\hline & & Lower & $79 \%$ \\
\hline
\end{tabular}

Overall, for greyscale pairs that were equiluminant, participants chose the greyscale that was darker on the left side $62.5 \%$ of the time. Accuracy scores were also calculated to examine how accuracy was influenced by luminance difference (see Table 3-1). When there was a 250 pixel difference between the greyscales, participants were accurate in choosing the greyscale that was actually darker $63 \%$ of the time. When there was a 500 or 1000 pixel difference between the greyscales, participants were accurate in choosing the greyscale that was actually darker $72.5 \%$ and $79.5 \%$ of the time. As VF differences were observed, accuracy scores were also compared across the upper and bottom VFs for each luminance difference. There was no significant difference in accuracy scores for 250 pixel differences $(t(38)=1.69, p=.08)$; however a difference was seen in both the 500 pixel $(t(38)=-1.83, p=.04)$ and the 1000 pixel luminance differences $(t(38)=-2.02, p=.03)$. Participants were more accurate in the upper VF in both instances $(500$ 
pixels: upper $=75 \%$, lower $=71 \% ; 1000$ pixels: upper $=83 \%$, lower $=79 \%$ ); however this difference only occurred in extrapersonal space as there were no accuracy differences in peripersonal space (all $p$ 's $>$.15).

\section{Discussion}

A significant leftward bias was observed, with participants exhibiting a stronger leftward bias in the lower VF than the upper VF. Interestingly a significant interaction was observed with length and both viewing distance and VF; however no consistent pattern of findings emerged. There were no space-based differences when comparing performance in peripersonal and extrapersonal space. This is consistent with prior suggestions that when the visual angle of stimuli is held constant, space-based differences will not occur (i.e., Dellatolas et al., 1996). Although prior investigations of perceptual asymmetries have identified the possibility of spacebased differences, the current findings did not demonstrate any such difference. Although variations in the type of task might also contribute to the lack of a significant difference, the current result supports the suggestion that when visual angle is maintained, space-based differences will not occur.

As prior studies have identified distance-based differences using line bisection tasks, it is possible that line bisection and the greyscales task are not measuring the same phenomenon. In the past, researchers (i.e., Nicholls et al., 1999) have failed to observe a correlation between the two tasks, which might show that performance on one task is not closely related to performance on the other. Although it remains unclear as to why the two phenomena do not correlate, motor involvement in manual line bisection may contribute to differential findings. The underlying neural mechanisms are thought to be similar for both tasks as both are proposed to measure 
spatial biases; however pseudoneglect previously observed on line bisection tasks might not be the same as the perceptual asymmetries measured by the greyscales task.

There was a significant difference observed between the VF conditions, with a stronger leftward bias being observed in the lower VF than in the upper VF. This was not consistent with the findings of McCourt and Jewell (1999) or McCourt and Garlinghouse (2000), who found a stronger leftward bias in the upper VF on a landmark task. This result, however, is consistent with that of Barrett et al. (2000), who also observed a stronger left bias in the lower VF. It is of interest to note that McCourt and colleagues $(1999 ; 2000)$ used tachistoscopic presentation (150 msec) in both studies and the method employed by Barrett et al. (2000) and in the current study was a free-viewing presentation. Methodological differences in task and presentation time appear to be the most likely explanation for these mixed results; however the method employed by Barrett et al. (2000) bears no similarities to the current method, further complicating the interpretation of these findings.

Examination of the accuracy scores by VF showed that participants were more accurate in the upper VF in extrapersonal space as the luminance difference increased. This suggests an upper VF accuracy advantage is occurring in extrapersonal space, which would be consistent with a stronger leftward bias in the lower VF (performance is not as accurate). As perceptual biases exhibited VF differences in the absence of distance-based differences it does not appear to be the case that distance-based and VF differences correspond with one another in perceptual asymmetries. Based on the current results, it would appear that this explanation is not plausible in explaining VF differences. As there is some evidence to indicate that dorsal and ventral visual stream differences might relate to VF processing, it would be of interest to compare perceptual asymmetries performance using tasks that specifically target either the dorsal or the ventral 
visual stream. This would assist in determining whether visual stream processing differences relate to the VF differences that have been observed in perceptual asymmetries.

An additional possibility relates to the more recent evolutionary explanations that have emerged from the examination of perceptual asymmetries in birds (Diekamp et al., 2005; Regolin, 2006). Further research in this area might also contribute to the understanding of VF differences that have been observed among human participants. As research in this area has been preliminary thus far, it is difficult to suggest possible mechanisms that might underlie the leftward bias. It has been suggested that processing differences in the upper and lower VFs might correspond with situations in which an individual would have been more likely to engage with a particular stimuli in a specific VF. Future research in this area should aim to clarify this through the identification of stimuli-specific processing differences in the upper and lower VFs. 


\section{CHAPTER 4 \\ PERCEPTUAL ASYMMETRIES IN GREYSCALES: OBJECT-BASED VERSUS SPACE- BASED INFLUENCES}

\section{Introduction}

Hemispatial neglect most typically occurs following right hemisphere damage, leading to a rightward spatial bias, which is believed to be the result of the right hemisphere's role in spatial attention (Heilman, Watson, \& Valenstein, 2003). Interestingly, left and right are only meaningfully defined with respect to a particular frame of reference (Subbiah \& Caramazza, 2000). When referring to left and right, their actual locations will differ depending on whether one is referencing their own body, or an object or location in space. A distinction has been made between retinocentric (Hillis \& Caramazza, 1995), body-centred (Heilman, Bowers, \& Watson, 1983), space-based (Halligan, Fink, Marshall, \& Vallar, 2003), and object-centred (Driver \& Halligan, 1991; Hillis \& Caramazza, 1995) hemispatial neglect. This illustrates the different frames of reference that are relied upon when discussing location and also how a disorder of one frame of reference does not necessarily indicate all will be affected in the same way.

Hemispatial neglect can also occur following left brain damage, although it is reported less frequently (Kleinman et al., 2007). It may be the case that right hemispatial neglect is underdiagnosed or misdiagnosed, leading it to be studied less and to remain poorly understood. Due to a lack of accurate data regarding the occurrence of right hemispatial neglect, Kleinman et al. (2007) examined 47 patients following left hemisphere stroke. They found that 19 percent of patients presented with right hemispatial neglect and that neglect was better detected when several testing measures were used. This illustrates that although neglect can be seen following left hemisphere stroke, it occurs less frequently than after right hemisphere damage. 
Object-based neglect has been examined by presenting patients with either an array of objects or a single stimulus and having them copy it (Gainotti, Messerli, \& Tissot, 1972). Objectbased neglect is seen when the left side of an object is neglected, regardless of where it is located or whether the image is presented in any particular orientation (Caramazza \& Hillis, 1990;

Driver, Baylis, \& Rafal, 1992; Driver \& Halligan, 1991; Niemeier \& Karnath, 2002). The central axis of the stimulus defines the left and right sides, which leads patients to neglect the left half of a specific object, but not that side of space more generally (Driver, Baylis, Goodrich, \& Rafal, 1994; Driver \& Halligan, 1991). As seen in Appendix K, when stimuli are presented in their upright orientation, object- and space-based coordinates are congruent and it is impossible to dissociate the two. When the object is rotated by $45^{\circ}$ (see Appendix K, Images b \& c), spaceand object-based coordinates can now be dissociated from one another, making it possible to demonstrate which of the two contributes more to the bias.

Driver and Halligan (1991) showed patient PP two nearly identical nonsense objects with obvious midlines, one directly above the other. The patient was asked to detect the difference between the two images in both an upright presentation and after $45^{\circ}$ rotation. Object-centred neglect was revealed as the principal axis of the shape, and not its spatial location, determined which portion would be neglected (Driver and Halligan, 1991). Object-based biases have been shown using similar tasks (Driver et al., 1994; Hillis, Rapp, Benzing, \& Caramazza, 1998) as well as reading tasks, where a portion of each individual word is neglected (Caramazza \& Hillis, 1990; Hillis \& Caramazza, 1995). In contrast to this, space-based neglect occurs when patients fail to orient toward or attend to the contralateral side of space (typically the left side; see Heilman et al., 2003 for a review), regardless of individual objects. 
Hemispatial neglect has been reliably identified using the greyscales task (Mattingley et al., 2004; see Appendix D). In choosing which image appears to be darker (or brighter), the image with the salient feature on the right is chosen the majority of the time, despite the images being equiluminant (Mattingley et al., 2004). This task has also been used to examine perceptual asymmetries in neurologically normal individuals, who tend to select the image with the salient feature on the left side (Nicholls et al., 1999). Similarly, participants typically bisect lines to the left of centre in manual (Barrett et al., 2000; Luh, 1995) and computerized line bisection (McCourt \& Jewell, 1999), and most often falsely indicate that the left end of a line is longer on the landmark task (Dufour et al., 2007). It has been suggested that the leftward bias exhibited by neurologically normal individuals, and referred to as pseudoneglect (Bowers \& Heilman, 1980; Jewell \& McCourt, 2000), from the same neural mechanisms as the rightward bias in hemispatial neglect (Loftus, Vijayakumar, \& Nicholls, 2009; McCourt \& Jewell, 1999).

Numerous explanations have been put forth to account for the leftward bias, such as premotor/intentional biases (Brodie \& Pettigrew, 1996; Heilman \& Valenstein, 1979) or scanning and reading habits (Chokron et al., 1998; Manning et al., 1990). These explanations are not adequate as a left bias is also seen among right-to-left readers and when using bimanual responding (Nicholls \& Roberts, 2002). An underlying asymmetry of spatial attention, resulting from right hemisphere dominance, could account for the leftward bias observed among neurologically normal participants as well as the rightward error exhibited by hemispatial neglect patients. The posterior parietal area in the right hemisphere is primarily responsible for spatial attention (e.g.s, Corbetta et al., 1995; Posner \& Petersen, 1990; Posner \& Rothbart, 2007), implying that attention is preferentially directed to the left side. This suggestion has been supported with neuroimaging evidence showing that information in the left VF activates 
proposed visuospatial attention networks more strongly than information in the right VF (SimanTov et al., 2007). Further, the right hemisphere is more active during line bisection and landmark tasks (Çiçek et al., 2009; Fink et al. 2000, 2001; Foxe et al., 2003), suggesting an attentional bias underlies pseudoneglect.

If the same neural mechanisms are responsible for both phenomena, the body-centred, object-based and space-based biases described in clinical neglect likely also contribute to the spatial biases observed among neurologically normal individuals. Prior research has examined each of these spatial frames of reference in neurologically normal individuals; however their contributions to the left bias are unclear as no consistent pattern of findings has emerged. This indicates the importance of further considering the influence of each frame of reference on the strength of the left bias.

Nicholls, Mattingley, Bradshaw, and Krins (2003) manipulated object-, trunk- and headcentred coordinates to determine their contributions to lateral biases. They found a leftward bias in all conditions with no effect of either trunk- or head-centered coordinates. The authors concluded that either object-based or space-based coordinates could be responsible for the left bias as only object-based coordinates were examined and the potential influence of space-based biases could not be excluded.

A bias toward the upper features of vertical stimuli has been observed on line bisection (Bradshaw et al., 1985; Drain \& Reuter-Lorenz, 1996; McCourt \& Olafson, 1997). Both leftward and upward biases have been explained in relation to object-centred biases as the most significant features are attended to, with the left side being significant due to Western reading and scanning habits and upper features carrying more information than the lower ones 
(Jeerakathil \& Kirk, 1994). This has been demonstrated by showing a bias toward the label "top", even when the labelled portion is not the actual top (Jeerakathil \& Kirk, 1994).

Nicholls et al. (2004b) employed the greyscales task and rotated each pair $45^{\circ}$ (see Appendix K, Images b \& c) to examine whether vertical and horizontal biases interact to influence the left bias. They found a significant leftward bias when greyscales were rotated negatively (see Appendix K, Image b); however no significant bias was seen when rotated positively (see Appendix K, Image c). Nicholls et al. (2004b) concluded that upper and leftward biases opposed one another in the positive rotation, eliminating both biases, whereas biases were additive in the negative rotation, leading to an upper left bias.

Nicholls et al. (2006b) used vertical (see Appendix K, Image d) and horizontal (see Appendix K, Image a) presentations of the greyscales task and manipulated head-centred coordinates. Participants showed significant leftward and upward biases only when their head was upright, showing that environment- and head-centered coordinates were co-dependent. Horizontal presentations of the greyscales were more affected by body-centred coordinates as "up" and "down" tend to be more objectively defined than "left" and "right". Nicholls et al. (2006b) concluded that body- and environment-centred coordinates mix in a destructive way, leading the left bias to be eliminated when participants tilted their head. Nicholls et al. (2006b) conducted a second experiment where tasks were completed in a supine position. When environmental up and down coordinates were inconsistent with stimulus-based up and down, the upward bias was eliminated; however, the left bias was not affected by body position. This showed that upper and leftward biases were dissociated, with the left bias occurring in relation to body-centred coordinates, and the upper bias being associated with environmental coordinates. 
Pia, Neppi-Modona, and Folegatti (2010) investigated whether the left bias could be seen in a solely object-based frame of reference. Participants bisected elongated caricatures of basset hounds, with some seeing images with the head on the left side and the tail on the right and others seeing the opposite. During the final bisection trials, the image was mirror-reversed to examine whether bisection errors would reverse as well. Results indicated that a rightward bias emerged on the mirror-reversed trials, showing it was possible to induce an object-centred bias on the newly learned canonical orientation of non-verbal visual stimuli.

Nicholls, Hughes, Mattingley, and Bradshaw (2004) compared space- and object-based coordinates directly (see Appendix K, Image e \& f) and also included the typical presentation (baseline condition) of the greyscales task. Findings indicated the congruent and baseline conditions exhibited the same bias, with no bias emerging in the incongruent condition. The authors concluded that object- and space-based biases were not additive as the bias in the congruent condition was not stronger than that observed in the baseline condition. It is possible that the baseline condition was spatially congruent as opposed to spatially neutral, accounting for the similar bias in these two conditions.

Orr and Nicholls (2005) also investigated the interaction of object- and space-based coordinates (see Appendix K, Image g), but used side-by-side presentation as opposed to parallel. Biases were maximal when objects were further apart from one another, indicating that the relative location of each image influenced the strength of the left bias. Results showed there was a bias toward the most centrally located stimulus (i.e., the inner object), indicative of an object-based bias that was dependent on the centrality of the object. Orr and Nicholls (2005) concluded that a stronger space-based central bias moderates a weaker object-based leftward 
bias, suggesting that both a moderate attentional gradient biased to the left side and a slightly stronger attentional bias to the central VF occur.

As both object- and space-based biases have been found (Nicholls et al., 2003; 2004a; 2006b; Orr \& Nicholls, 2005), and it is not clear whether they interact with or counteract one another, two studies were conducted to examine the contributions of both object- and spacebased coordinates. The current work also examined how the relative position of the images within the display influenced the strength of the leftward bias. The current studies were conducted to determine whether one frame of reference is relied upon in a consistent manner, or whether this is dependent upon context. It was believed space-based coordinates would contribute more strongly when greyscales were used; however object-based biases would emerge more strongly when actual objects were used in place of the greyscales, which are more ambiguous.

\section{Study 1}

The first study investigated the interaction of object- and space-based biases by shifting one greyscale in each pair toward either the left or the right side. As the centrality of the image and the location of one rectangle relative to the other have shown an influence on the leftward bias (Orr \& Nicholls, 2005), relative position was manipulated to see how the proximity of the greyscale rectangles impacted the strength of the left bias. The rectangles were presented parallel to one another, with either a partial overlap (50\% of each rectangle) or no overlap. As relative position has typically not been considered (Nicholls et al., 2003; 2004a; 2004b; 2006b), either by omission or by collapsing across seemingly similar conditions, no directional hypotheses were made in relation to this factor. 
Although it would seem logical to expect that the leftward bias would be strongest when the relevant portion was shifted toward the upper left quadrant and would be weakest (or absent) when shifted toward the lower right quadrant (as in Nicholls et al., 2004b), an additive bias such as this has not typically been found (Nicholls et al., 2004a; 2006b). Following Orr and Nicholls (2005), it was expected that strong attentional biases to the centre VF and moderate leftward object-based biases would both occur. It was expected that incorporating relative position would reveal that collapsing across spatial conditions masks the complex interaction of relative position and object- and space-based coordinates in relation to lateral biases.

\section{Method}

Participants. Forty undergraduate Psychology students (11 males; mean age $=18.95, S D$ $=1.26)$ at the University of Saskatchewan participated in the current study. Students received course credit in exchange for participation. Based upon self-report (see Appendix A; Elias et al., 1998), four participants were left-handed and all participants had normal or corrected-to-normal vision. This study was conducted with the ethical approval of the Behavioural Research Ethics Board at the University of Saskatchewan and has therefore been performed in accordance with the ethical standards laid down in the 1964 Declaration of Helsinki.

Materials. Stimuli were administered on an IBM clone computer (PIV 2.6 GHz) interfaced with a 19" LCD monitor running at 1024 x 768 resolution. The maximum resolution of the monitor was 1400 x 1050 and the refresh rate was 8 ms. E-prime (Psychology Software Tools, Inc., Pittsburgh, USA; www.pstnet.com/eprime) was used to administer a series of 288 greyscales measuring $154 \mathrm{~mm}$ in length. Stimuli consisted of mirror-reversed image pairs, with one of the images being dark on the left side and the other dark on the right. There were nine location conditions (see Appendix L), with one being the typical presentation where the two 
images are presented one directly above the other in the centre of the VF (see Appendix L, Image 1a). There were two conditions in which the bottom greyscale (of the pair) was shifted toward the left side: one where the two images were partially overlapping (see Appendix L, 1b) and one with no overlap (see Appendix L, 1c). There were also two conditions where the top greyscale was shifted toward the left side: one where the two images were partially overlapping (see Appendix L, 1d) and one with no overlap (see Appendix L, 1e). Each of these conditions was repeated with the greyscales being shifted toward the right side (see Appendix L, 1f, 1g, 1h \& 1i). The overlap always represented 50 percent of the length of the rectangle, and when rectangles were not overlapping both rectangles were shifted, one toward each side (neither was in the actual centre of the VF). The side of the rectangle that was bright was counterbalanced so that it occurred on both the left and right sides of both the top and bottom rectangles.

All presentations were free-viewing and visible for a maximum of five seconds. Participants were asked to select whether the image on the top or the bottom of the pair appeared to be darker. Responses were made using the number pad keys 8 and 2, with 8 representing the top image being chosen and 2 representing the bottom image. As simple button press and keyboard responses evoke a minimal amount of motor movement (i.e., McCourt \& Olafson, 1997), responses were not confounded by pre-motor or motor biases. Responses, as well as response times, were recorded. A response bias score was calculated by subtracting the number of leftward responses from the number of rightward responses, with a negative score indicating a leftward bias (Nicholls et al., 1999). An analysis of variance was used to determine whether three factors relating to spatial location influenced the leftward bias: the horizontal direction in which the image was shifted (left or right), which rectangle of the greyscale pairing had the dark portion on the left (upper, lower) and the location of one greyscale rectangle relative to the other 
(overlap, no overlap). There was no expected main effect for location of the image within the image pair; however it was thought that this factor would interact with the horizontal direction of shift, accounting for its inclusion in the analysis.

Procedure. Following informed consent (see Appendix M), participants completed a demographic questionnaire addressing items such as, sex, age, visual or hearing impairments, and handedness and footedness (see Appendix A; Elias et al., 1998). Following completion of the questionnaire, participants completed the greyscales task. Trials were presented in random order during the task. All participants were debriefed following participation (see Appendix N).

\section{Results}

A one-sample $t$-test was used to examine whether a bias was seen on the centrally presented greyscales (typical bias). A significant leftward bias was observed, $t(39)=-6.434$, $p<.001, d=.718(M=-12.15, S D=11.94)$, confirming the expected bias in this condition. A onesample $t$-test was also used to examine whether a bias was seen for the overall sample when collapsing across all spatial location conditions. A significant leftward bias was observed, $t(39)=$ $-5.243, p<.001, d=.643(M=-76.00, S D=91.68)$, confirming that when spatial location is not considered a leftward bias is seen.

A $2 \times 2 \times 2$ repeated-measures ANOVA was computed with within-subjects independent variables of location within the image pair (upper, lower), direction of horizontal shift (left, right) and relative position (overlap, no overlap), with the dependent variable being bias score on the greyscales task. As expected, the main effect of location within the image pair was not significant, $F(1,39)=.058, p=.811, \eta_{\mathrm{p}}{ }^{2}=.001$, showing that there was no response bias based solely on whether the image chosen was the top $(M=-7.75, S D=10.01)$ or bottom $(M=-7.88$, $S D=10.40)$ rectangle of the pair. There was a main effect of relative position, $F(1,39)=9.871$, 
$p=.003, \eta_{\mathrm{p}}{ }^{2}=.202$, indicating a stronger leftward bias when stimuli were partially overlapping $(M=-9.22, S D=10.43)$ compared with no overlap $(M=-6.41, S D=10.47)$. There was also a main effect of horizontal shift, $F(1,39)=30.672, p<.001, \eta_{\mathrm{p}}{ }^{2}=.440$, indicating a stronger leftward bias when stimuli were shifted toward the right side $(M=-13.01, S D=9.93)$ compared with the left side $(M=-2.63, S D=13.19)$.

These main effects were qualified by a significant horizontal shift by relative position interaction, $F(1,39)=11.545, p=.002, \eta_{\mathrm{p}}{ }^{2}=.228$ (see Figure 4-1). Post hoc paired-samples $t$-tests indicated that when stimuli were partially overlapping on the right side there was a stronger leftward bias than when there was no overlap, $t(39)=5.256, p<.001$. No differences were seen between these two conditions on the left side, $t(39)=-.549, p=.586$.

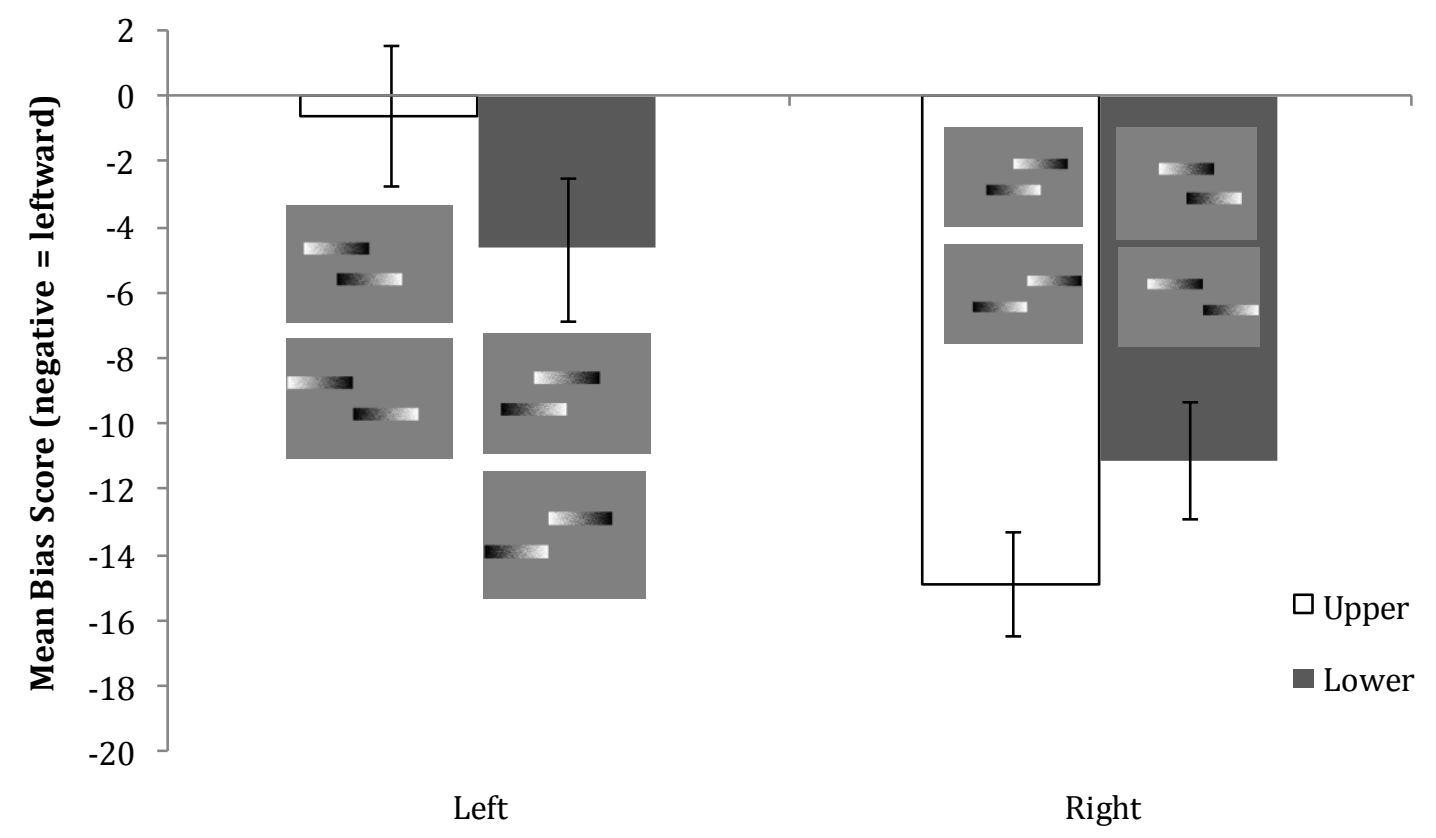

Horizontal Dimension

Figure 4-1. Interaction of horizontal shift and relative position. Error bars indicate the standard error of the mean.

There was also a significant horizontal shift by vertical location within the image pair interaction, $F(1,39)=14.261, p=.001, \eta_{\mathrm{p}}{ }^{2}=.268$ (see Figure 4-2). A significant leftward bias was 
seen in all conditions (upper right: $t(39)=-9.378, p<.001$; lower right: $t(39)=-6.261, p<.001$; lower left: $t(39)=-2.136, p=.039)$, except for upper images shifted toward the left $(t(39)=-.280$, $p=.781$ ). Post hoc paired-samples $t$-tests indicated that stimuli shifted to the upper right side had the strongest leftward bias, followed by those shifted to the lower right, the lower left, and then the upper left. All conditions differed significantly from one another (comparison of conditions as listed above: $t(39)=-3.112, p=.003 ; t(39)=2.899, p=.006 ; t(39)=3.595, p=.001)$.

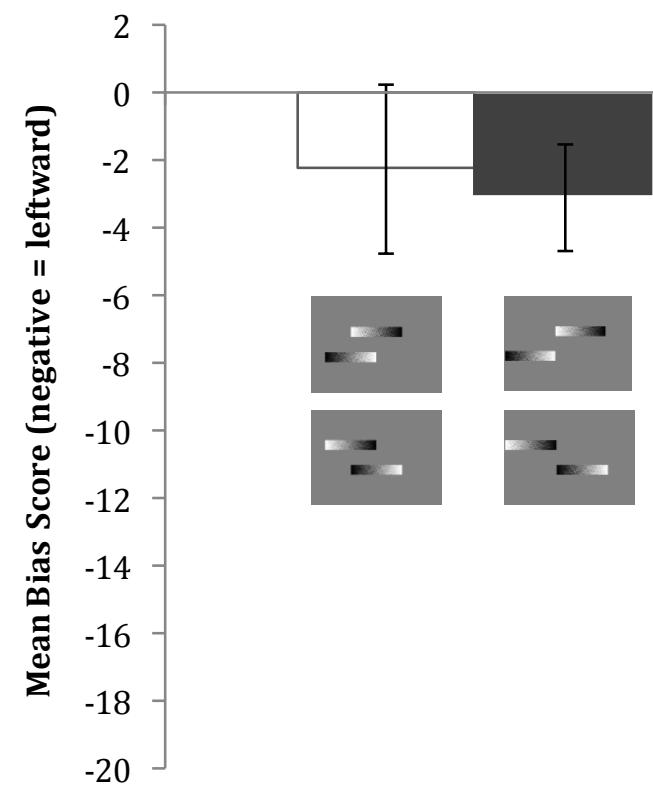

Left

Horizontal Dimension

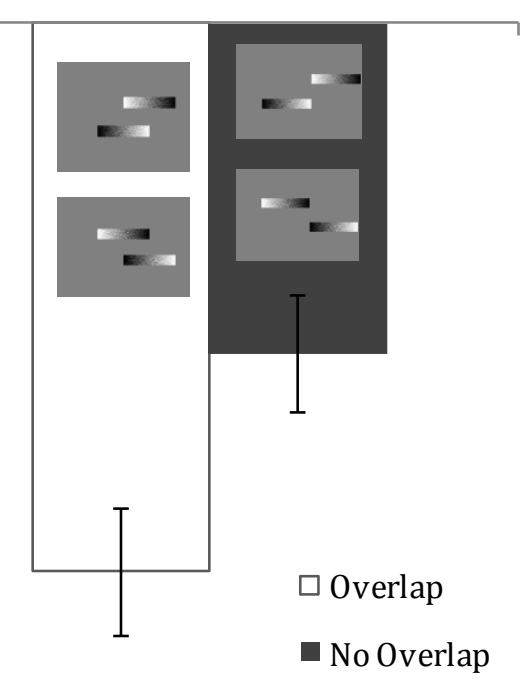

Right

Figure 4-2. Interaction of horizontal shift and vertical location. Error bars indicate the standard error of the mean.

\section{Discussion}

A leftward bias was observed for greyscales presented in the typical orientation and for the overall sample when collapsing across spatial conditions. As there was no bias in the upper left quadrant, upper and left biases were not additive, which is consistent with prior research (Nicholls et al., 2004a; 2006b). The current findings support the occurrence of a leftward objectbased bias moderated by an attentional bias to the centre VF (Orr \& Nicholls, 2005). 
The presence of a stronger left bias on the right side indicates that a leftward object-based bias occurred and that leftward space-based biases were not strong enough to overpower the object-based bias. The occurrence of a leftward bias when images were shifted to the right side could indicate that the centremost dark portion was chosen, indicating a centre VF bias. Orr and Nicholls (2005) observed a centrality bias and concluded that a stronger space-based central bias moderates a weaker object-based leftward bias. This would explain the occurrence of a leftward object-based bias when images were shifted to the right side as the left side of both rectangles was more central than in the left shift condition.

The occurrence of a strong centrality bias, which moderates a leftward object-based bias, could also explain the lack of a leftward bias for images shifted toward the upper left. If the centrality bias opposed the leftward object-based bias, the left bias should be weakened, particularly when the salient portion of the image is located on the extreme left. It might be the case that the left object-based bias was diminished by a bias to the centre VF, leading to no bias in this condition. Object-based coordinates showed a clear influence; however when object- and space-based coordinates were congruent, a leftward bias was only seen when the shifted image was located in the lower VF.

The occurrence of a stronger left bias in the lower VF is consistent with previous research on the greyscales task (Thomas \& Elias, 2010) and on line bisection (Barrett et al., 2000), although object-based biases were not considered in either of these studies. The bias appears to be strong enough to allow for a leftward object-based bias to emerge in the lower VF only, and not in the upper VF. Both object- and space-based biases likely interact with upper and lower VF location; however the context in which either bias predominates remains unclear 
When greyscales were shifted toward the right side, the leftward bias was stronger when images were partially overlapping (located more closely together). In contrast, Orr and Nicholls (2005) observed that the left bias was strongest when greyscale rectangles were located further away from one another. Results from both studies point to the importance of considering proximity when examining perceptual asymmetries. Although these results contradict one another, it is important to bear in mind that the methodologies employed in each study were not identical. Orr and Nicholls (2005) presented images side-by-side and we presented them parallel to one another, which could have contributed to the differing results. Further, the interaction observed in the present study indicates that the proximity of the two images not only influences the strength of the leftward bias, but that proximity influences the left bias differently in the left $\mathrm{VF}$ and the right VF.

The greyscales task has been used reliably to demonstrate leftward spatial biases (i.e., Nicholls et al., 1999; 2003; 2006b; Thomas \& Elias, 2010); however this task is not optimal for studying object-based biases as the images do not have a clear central axis that can be used in defining the left side and the right side of objects. A second study was carried out to determine whether participants would show a leftward luminosity bias when viewing everyday objects, such as chairs or drinking glasses, which were symmetrical about their central axis.

\section{Study 2}

It has been demonstrated in hemispatial neglect patients that object-based biases emerge when objects with a clear midline are used (Driver et al., 1994; Driver \& Halligan, 1991) in place of lines or the greyscales. Therefore, actual objects, which were symmetrical about the midline, were used to further examine how object- and space-based coordinates contribute to perceptual asymmetries. Participants were to select which object appeared brighter overall and it was 
expected that the image that was bright on the left side would be chosen most often. The bias on this task was expected to be similar to that observed on the greyscales task (Nicholls et al., 1999).

Three conditions were included: baseline (central presentation), congruent (VF and direction of luminosity were consistent) and incongruent (VF and direction of luminosity were inconsistent). Following Nicholls et al. (2004a), it was hypothesized that participants would show a left bias in both the baseline (central) and the congruent conditions of the object luminosity task. As Nicholls et al. (2004a) did not find additive object- and space-based biases, it was not expected that the bias would be stronger in the congruent condition. In the incongruent condition, it was expected that the left bias would be weaker or absent, illustrating the occurrence of both space- and object-based biases.

\section{Method}

Participants. Seventy-seven undergraduate Psychology students (31 males; mean age $=19.70, S D=1.47$ ) at the University of Saskatchewan participated in the current study. Students received course credit in exchange for participation. Based upon self-report (see Appendix A; Elias et al., 1998), all participants were right-handed and had normal or correctedto-normal vision. This study was conducted with the ethical approval of the Behavioural Research Ethics Board at the University of Saskatchewan and has therefore been performed in accordance with the ethical standards laid down in the 1964 Declaration of Helsinki.

Materials. Stimuli were administered on the same computer as experiment 1. E-prime was used to administer a series of images of everyday objects with a lateralized direction of luminosity. In block 1, participants viewed 12 mirror-reversed pairs of objects (see Appendix O), presented one directly above the other, with one object being bright on the top left and the other 
on the top right. All objects had a definitive midline and were symmetrical about that midline. Each image pair was presented twice to ensure that all images appeared as the top stimulus, leading to a total of 24 trials.

During the second block of trials, image pairs were presented in two of the four quadrants of the screen (top left, top right, bottom left, bottom right). Only one image was visible in each VF and images were presented diagonal to one another, creating two spatial location pairings: top left-bottom right (TL/BR) and bottom left-top right (BL/TR). Luminosity direction was either congruent with side of presentation (left side bright in left VF, right side bright in right $\mathrm{VF}$ ) or incongruent (left side bright in right VF, right side bright in left VF).

All presentations were free-viewing and visible for an unlimited amount of time. In both blocks of trials participants were asked to select whether the image on the top or the bottom of the pair appeared to be brighter. Responses were made using the number pad keys 8 and 2, with 8 representing the top image being chosen and 2 representing the bottom image. Responses, as well as response times, were recorded. For block one, a response bias score was calculated by subtracting the number of leftward responses from the number of rightward responses, with a negative score indicating a leftward bias (Nicholls et al., 1999). For block two, response bias scores were created (in the same manner) for each of the four presentation conditions. Separate bias scores were also calculated based on either object- or space-based coordinates. Object-based biases were determined based on whether the direction of luminosity in the chosen image was rightward or leftward. Space-based biases were determined based on whether the image chosen was located on the left side or the right side of space.

Procedure. Following informed consent (see Appendix P), participants completed a demographic questionnaire addressing items such as, sex, age, visual or hearing impairments, 
and handedness and footedness (see Appendix A; Elias et al., 1998). Following completion of the questionnaire, all participants completed trial block one, followed by trial block two. Trials were presented in random order during each block. All participants were debriefed following participation (see Appendix Q).

\section{Results}

A one-sample $t$-test was used to examine whether a bias was seen in the first block of trials (baseline) and a significant leftward bias was observed, $t(76)=-5.147, p<.001, d=.508(M=$ -3.66, $S D=6.24)$. The data were then analysed separately for space-based and object-based biases. A one-sample $t$-test examined the data for an overall left side spatial bias, which was not significant, $t(76)=-1.459, p=.149, d=.165(M=-4.52, S D=27.19)$, although the mean was in the correct direction. A one-sample $t$-test was also used to look for an object-based bias and a significant leftward bias emerged, $t(76)=-4.741, p<.001, d=.478(M=-13.56, S D=25.10)$. A paired-samples $t$-test was used to compare the strength of object- and space-based biases, which was significant $(t(76)=2.323, p=.023, d=.257)$, illustrating that object-based biases were stronger when actual objects were used.

A $2 \times 2$ repeated-measures analysis of variance was computed with within-subjects independent variables of spatial location (TL/BR, BL/TR) and direction of luminosity (congruent, incongruent) and the dependent variable being the bias score on the object

luminosity task. There was a main effect of spatial location, $F(1,76)=42.616, p<.001, \eta_{\mathrm{p}}{ }^{2}=.359$. One-sample $t$-tests were used to examine biases in the TL/BR and BL/TR spatial location conditions. A significant leftward bias was observed for BL/TR presentations, $t(76)=-5.862$, $p<.001(M=-6.52, S D=9.76)$, whereas a significant rightward bias was observed for TL/BR presentations, $t(76)=3.699, p<.001(M=4.26, S D=10.11)$. This is indicative of a lower VF bias as 
the image on the bottom left image was chosen more often in the BL/TR presentation and the image on the bottom right was chosen more often in the TL/BR presentation.

There was also a main effect of side of luminosity, $F(1,76)=22.473, p<.001, \eta_{\mathrm{p}}{ }^{2}=.228$. One-sample $t$-tests were used to examine biases in the congruent and the incongruent conditions. A significant leftward bias was observed for congruent presentations, $t(76)=-4.000, p<.001(M=$ -9.04, $S D=19.83$ ), whereas a significant rightward bias was observed for incongruent presentations, $t(76)=2.323, p=.023(M=4.52, S D=17.07)$.

The interaction of spatial location and direction of luminosity was also significant, $F(1,76)=11.596, p=.001, \eta_{\mathrm{p}}{ }^{2}=.132$, (see Figure 4-3). Post hoc paired-samples $t$-tests indicated that congruent presentations in the right VF had a stronger leftward bias than incongruent presentations in the right $\operatorname{VF}(t(76)=3.462, p=.001)$, or than either congruent $(t(76)=5.521$, $p<.001)$ or incongruent presentations $(t(76)=8.722, p<.001)$ in the left VF. As well, a stronger rightward bias was seen in the left VF on incongruent presentations compared to congruent presentations in either the left VF $(t(76)=-5.444, p<.001)$ or the right $\operatorname{VF}(t(76)=6.934, p<.001)$. The difference between congruent and incongruent presentation conditions in the left VF approached significance, $t(76)=1.707, p=.092$. 


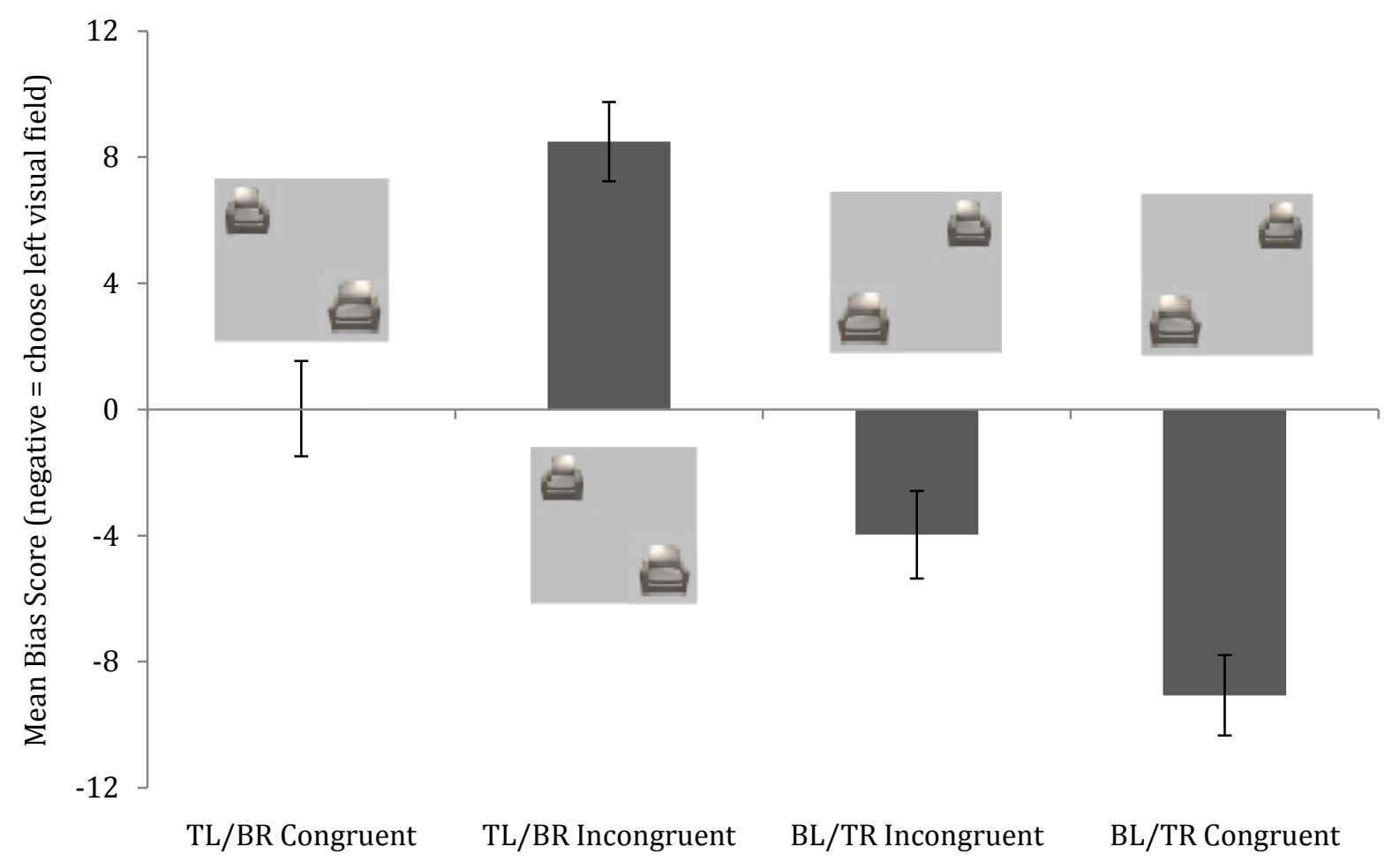

Figure 4-3. Interaction of side of space and side of luminosity. Error bars indicate the standard error of the mean.

\section{Discussion}

A leftward bias was seen for central presentations (baseline condition), suggesting that everyday objects with directional lighting exhibit similar biases to those seen on the greyscales task (Nicholls et al., 1999) and can be used to investigate object-based biases. Pia et al. (2010) demonstrated that object-based biases could be elicited for stimuli shortly after their orientation had been learned. The left side and the right side of the greyscale images are more ambiguously defined than are the left side and the right side of objects. The objects used in the current study had left and right sides that could be objectively defined by their central axis, creating optimal stimuli for examining object-based biases. Further, everyday objects provide a more ecologically valid way of examining the influence of object-based biases. Consistent with our hypothesis, the use of actual objects allowed for a strong object-based bias to be seen, whereas no overall spatial bias was seen. 
A leftward bias was seen for congruent trials, which is consistent with the findings of Nicholls et al. (2004a), and could be indicative of either object- or space-based biases. A rightward bias was observed for incongruent trials, which is consistent with the occurrence of a solely space-based bias (see Nicholls et al., 2004a). Interestingly, when the four possible conditions were compared individually, biases were not equivalent for the two congruent and incongruent conditions.

As seen in Figure 4-3, a leftward spatial bias was seen when images were presented in the BL/TR orientation, regardless of whether the presentation was congruent or incongruent. Further, a rightward bias was seen in the TL/BR orientation for the incongruent condition, but no bias was seen in the congruent condition. Two conditions exhibited a left side spatial bias in that the image in the left VF was chosen most often (both BL/TR presentations). In the TL/BR incongruent condition the image in the right VF was selected most often, which was indicative of a leftward object-based bias. A left object-based bias was also seen in the BL/TR congruent condition as space- and object-based biases were consistent with one another and it was not possible to determine which one led the bias to emerge.

The incongruent conditions exhibited opposite patterns of results in that participants showed a leftward object-based bias in one condition and a leftward spatial bias in the other. Both object- and space-based biases are clearly occurring and it appears that there may be an attentional bias toward the lower VF that interacts with these biases. This is consistent with the results from experiment 1 as well as with prior work showing a stronger left bias to the lower VF (Barrett et al., 2000; Thomas \& Elias, 2010). The image chosen in each condition with a bias was the image presented in the lower VF. In the condition with no bias, the image in the lower VF 
was both lit from the right and in the right VF, suggesting the lower VF image must have either leftward space-based or leftward object-based coordinates for a bias to emerge.

\section{General Discussion}

Results from both studies are consistent with previous research in that there was a significant leftward bias for central presentations where one image was directly above the other. When greyscales were used in Experiment 1, a leftward bias was seen when all spatial location conditions were collapsed together. This illustrates that when stimuli that are preferential to spatial biases are used, a spatial bias is seen. In Experiment 2, a leftward object-based bias was observed when comparing across all conditions, showing that when objects were used, objectbased biases were stronger. It is of interest to note that, although the objects used in study 2 were visually more complex than the rectangles employed in Study 1, the effect size for the spatial bias on the greyscales measure was larger than the object bias for the object luminosity task. Although the use of actual everyday objects led object-based biases to emerge more strongly, it appears that visuospatial tasks, which elicit spatial biases, show slightly stronger biases overall.

When each condition was examined separately, it could be seen that both space- and object-based coordinates contribute to the leftward bias, but the way in which they do so is not straightforward. As demonstrated, a leftward bias is seen when all conditions are considered simultaneously and in separating out each spatial location condition it is revealed that object- and space-based biases interact to influence the strength of the left bias. The current results support the findings of Orr and Nicholls (2005) that a strong centrality bias moderates a weaker leftward object-based bias. It was also shown that images presented in the upper and lower VFs are not equivalent, particularly on the left side. Prior work has shown that the left bias is stronger in the lower VF (Barrett et al., 2000; Thomas \& Elias, 2010), which could explain the absence of a bias 
in the upper left shift condition in experiment 1 . This also suggests that a centre VF spatial bias cannot entirely account for the left bias as it relates to spatial location.

The lower VF bias appears to be strong enough to favour responding toward the lower image on the left side, leading to no bias when the image is located on the upper left. The interaction of these two spatial biases becomes increasingly complex when the left VF and the right VF are considered separately. Experiment 2 clarified that both object- and space-based biases contribute and further, that using certain stimuli make it more likely that either object- or space-based biases will contribute more strongly. Therefore it would seem that the frame of reference that is primarily relied upon is dependent on the type of stimulus used and on context.

The location of one rectangle relative to the other was found to influence the strength of the left bias. When the greyscale rectangles are presented parallel, with one image being directly above the other, the influence of proximity cannot be examined. Previously it was shown that images that were located farther apart from one another showed a stronger left bias (Orr \& Nicholls, 2005). The current results showed a stronger left bias when images were located more closely together on the right side. Although results were inconsistent across studies, they indicate the importance of considering relative position in future research to better understand how proximity influences the left bias and further, how relative position interacts with left and right VF presentation.

The results from both studies relating to object- and space-based biases were similar, suggesting that the current results are not ambiguous, but alternatively they represent a reliable pattern of findings that emerges when stimuli targeting one type of bias are used and individual conditions are examined. When collapsing across similar conditions, these complex interactions are masked and it is only when each condition is examined separately that these effects emerge. 
In order to elicit either object- or space-based biases, one must use stimuli that will lead that type of bias to be preferential. Results illustrate that a bias toward stimuli located in the lower VF interacts with both object- and space-based biases, regardless of whether the greyscales or images of objects are used. Further, this interaction occurs such that the leftward bias is eliminated under certain conditions. Further research is needed to determine the precise conditions under which this occurs, but it is apparent that both object- and space-based biases influence the left bias and that their individual contributions are dependent on the stimuli used and their interaction with VF presentation. 


\section{CHAPTER 5 \\ UPPER AND LOWER VISUAL FIELD DIFFERENCES IN PERCEPTUAL ASYMMETRIES \\ Introduction}

It has been reliably demonstrated that neurologically normal individuals show a leftward spatial bias, referred to as pseudoneglect due to its similarity to clinical hemineglect (Bowers \& Heilman, 1980; Jewell \& McCourt, 2000). This phenomenon has been examined using a variety of tasks, such as line bisection (Luh, 1995; McCourt \& Jewell, 1999) and the landmark task (Dufour et al., 2007). Line bisection tasks typically demonstrate a leftward bias as individuals tend to bisect lines to the left of centre in both manual (i.e., Barrett et al., 2000; Luh, 1995) and computerized line bisection (i.e., McCourt \& Jewell, 1999; Rolfe, Hamm, \& Waldie 2008). The landmark task is a perceptual version of the line bisection task where participants are shown a pre-transected line and they are to indicate whether the left side or the right side of the line appears shorter (Milner, Harvey, Roberts, \& Forster 1993; Rueckert \& McFadden 2004). On the landmark task, participants falsely indicate that the right end of the line is shorter the majority of the time (Dufour et al., 2007, Milner et al. 1993; Rueckert \& McFadden 2004).

The greyscales task (Nicholls et al., 1999) has been used to investigate perceptual asymmetries in judgments of luminosity. Participants are shown a pair of mirror-reversed rectangles, where one is dark on the left side and the other is dark on the right, with each gradually becoming lighter on the opposite side. Participants tend to select the image that is dark on the left side as being darker overall, despite the images being equiluminant. Similar tasks assessing numerosity, size (Nicholls et al., 1999), and distance (Krupp et al., 2010), have been developed and participants tend to choose the image with the salient feature (i.e., larger, closer, etc...) on the left side most often on these measures as well. 
A number of potential explanations have been proposed to account for the leftward bias. It was suggested that scanning and reading habits (Chokron et al., 1998; Manning et al., 1990) or pre-motor/intentional biases (Brodie \& Pettigrew, 1996; Heilman \& Valenstein, 1979) might lead to perceptual asymmetries. However, Nicholls and Roberts (2002) demonstrated that although these factors might influence the left bias, they cannot account for it entirely as right-toleft readers and bimanual responding also lead to leftward biases. There has been some preliminary indication that perceptual asymmetries also occur in birds (Diekamp et al., 2005; Regolin, 2006; Rugani, Kelly, Szelest, Regolin, \& Vallortigara, 2010), as they demonstrate a leftward bias similar to that observed in humans. This suggests that they also show lateralization of attention, which could have an evolutionary basis (Diekamp et al., 2005; Regolin, 2006).

The explanation with the most support appears to be that of an attentional bias (Niemeier, Stojanoski, Singh, \& Chu, 2008). As the right hemisphere, specifically the posterior parietal area, is primarily responsible for spatial attention (e.g., Corbetta et al., 1995; Posner \& Petersen, 1990; Posner \& Rothbart, 2007), attention is preferentially directed toward the left side of space. This suggestion has been supported by neuroimaging data demonstrating that visuospatial attention networks are more activated by information in the left VF (Siman-Tov et al., 2007). Further, neuroimaging data indicate that the right hemisphere is more active during line bisection and landmark tasks (Bjoertomt et al., 2002; Çiçek et al., 2009; Fink et al., 2000; Fink et al., 2001; Foxe et al., 2003).

Although the left bias might be attentional in nature, a phenomenon that remains relatively unexplored is that of upper and lower VF differences in perceptual asymmetries. Upper and lower VF differences have been observed across a variety of other tasks (Christman \& Niebauer, 1997; Previc, 1990), with a lower VF bias emerging in many instances. Looking at 
motion processing, a lower VF advantage is seen for direction discrimination (Amenedo, PazoAlvarez, \& Cadaveira, 2007; Edwards \& Badcock, 1993), segmentation of moving targets (Lakha \& Humphreys, 2005), motion in depth (Regan, Erkelens, \& Collewijn, 1986), and centripetal motion processing (Edwards \& Badcock, 1993; Raymond, 1994). Across various studies, a bias toward the use of primarily lower VF information has been observed during locomotion (e.g., Foley \& McChesney, 1976; Guez, Gargasson, Rigaudiere, \& O'Regan, 1993; Murasugi \& Howard, 1989), with some indication of a similar lower VF bias in predatory behaviour among other species, such as the praying mantis (Prete, 1993). The lower VF advantage in motion perception is thought to relate to the role of the lower VF in visuomotor coordination and in guiding locomotion (Christman \& Niebauer, 1997).

There is also a lower VF advantage for temporal resolution as seen on the critical flicker fusion task (see Christman \& Niebauer, 1997 for a review). There is some evidence for better colour matching abilities in the left lower VF (Pennal, 1977), but these differences might simply relate to temporal processing. Rezec and Dobkins (2004) report a lower VF advantage when searching for a target amongst distracters, referred to as an attentional weighting bias. A lower VF advantage has also occurred for reaction time performance (Payne, 1967), for spatial resolution (Carrasco, Williams, \& Yeshurun, 2002; Rezec \& Dobkins, 2004), in the perception of illusory contours (Rubin, Nakayama, \& Shapley, 1996), and for contrast sensitivity at low to moderate spatial frequencies (Cameron, Tai, \& Carrasco, 2002; Carrasco et al., 2002; Lundh, Lennerstrand, \& Derefeldt, 1983; Rijsdijk, Kroon, \& van der Wildt, 1980). An overestimation of perceived spatial frequency has been shown in the left lower VF (Edgar \& Smith, 1990). It has also been shown that effects regarding temporal and spatial resolution interact with one another (see Christman \& Niebauer, 1997 for a review); however no clear pattern of results has emerged. 
Visual search studies have shown both right and upper VF advantages (Efron, Yund, \& Nichols, 1987; 1990; Previc \& Blume, 1993). Overall, tasks demonstrating an upper VF advantage have also shown a right VF advantage, whereas those showing a lower VF advantage demonstrate a left VF advantage (Christman \& Niebauer, 1997). Both upper and right VF advantages are seen for local processing, visual search, and categorical judgments, such as object identification, whereas lower and left VF advantages are reported for global processing and coordinate spatial judgments requiring visuomotor coordination, and global motion (Christman, 1993; Kosslyn, 1987; Niebauer \& Christman, 1998; Previc, 1990). Interestingly, these findings showed that left-right differences were confined to the lower VF and upper-lower differences only occurred in the left VF, suggesting a functional linkage between the lower and left VFs and the upper and right VFs (Christman \& Niebauer, 1997).

Prior research has demonstrated a similar relation in visuospatial attention as hemispatial neglect is most pronounced in the left and lower VFs (Rubens, 1985). Hemispatial neglect is typically characterized by a rightward orienting bias; however in many cases a lower VF bias also occurs (Rubens, 1985). There is also evidence that horizontal and vertical neglect interact, making neglect most severe in the lower left VF. Few studies to date have examined upper and lower VF differences in perceptual asymmetries using horizontal stimuli. McCourt and Garlinghouse (2000) and McCourt and Jewell (1999) investigated potential VF differences using computerized line bisection. Participants were presented with pre-transected lines at $3.6^{\circ}$ and $5.8^{\circ}$ visual angle (respectively) either above or below the midline. Lines were presented tachistoscopically $(150 \mathrm{msec})$. Participants identified the location of the transector with respect to the perceived midpoint of the line (to the left or right of veridical centre). In both studies, 
McCourt and colleagues found a stronger leftward bias when the stimuli were located in the upper compared to the lower VF.

Barrett et al. (2000) examined upper and lower VF differences using free-viewing manual line bisection (unlimited time to complete). Line bisection was performed at $40.5 \mathrm{~cm}$ either above or below the horizontal plane, meaning that the actual distance between the upper and lower VF presentations was much larger than in the task used by McCourt and colleagues (1999; 2000). In contrast to the findings of McCourt and colleagues, Barrett et al. found a stronger leftward bias in the lower VF as opposed to the upper VF. Thomas and Elias (2010) also found a stronger leftward bias in the lower VF on the greyscales task using a free-viewing presentation. Methodological differences might explain the mixed findings observed in prior research. Both presentation time and task type differed between the four previous studies. Prolonged presentation allows stimuli to be examined more thoroughly, whereas brief presentation times (e.g., $150 \mathrm{msec}$ ) do not allow for saccadic eye movements (e.g., Nagel-Leiby, Buchtel, \& Welch, 1990). Although it has been shown that covert scanning is not a suitable explanation (McCourt \& Jewell, 1999), it remains possible that presentation time influences subsequent judgments regarding the images. Eye tracking provides a useful way to examine which specific portions of the screen are focused on during task completion and whether eye movements are consistent with subsequent responses.

The purpose of the present study was to clarify whether upper and lower VF differences would be seen on the greyscales task, as there has been a lack of prior research examining VF differences. We investigated whether presentation time and VF interacted to influence the strength of the left bias. As prior findings have been inconsistent, upper and lower VF presentations were compared using the greyscales task (Nicholls et al., 1999). Length of time 
allotted to examining stimuli was manipulated by including both brief $(150 \mathrm{msec})$ and prolonged $(10 \mathrm{sec})$ viewing conditions. As $150 \mathrm{msec}$ presentation time does not allow for saccadic eye movements or scanning (Nagel-Leiby et al., 1990), eye tracking was used during prolonged trials to examine where participants were looking and whether eye movements were consistent with participant responses. It was expected that VF would interact with presentation time and that participants would exhibit a stronger leftward bias in the upper VF when presentation was brief (McCourt \& Garlinghouse, 2000; McCourt \& Jewell, 1999) and a stronger leftward bias in the lower VF when presentation was prolonged (Barrett et al., 2000; Thomas \& Elias, 2010).

\section{Method}

\section{Participants}

Forty-three undergraduate Psychology students (11 males; age: $M=20.21, S D=4.66$ ) at the University of Saskatchewan participated in the current study. Handedness was determined using the Waterloo Handedness Questionnaire-Revised (see Appendix A; Elias et al., 1998), which indicated 37 participants were right-handed and 6 were left-handed. Students received course credit in exchange for participation. Based upon self-report, all participants had normal or corrected-to-normal vision. This study was conducted with the ethical approval of the Behavioural Research Ethics Board at the University of Saskatchewan and has therefore been performed in accordance with the ethical standards laid down in the 1964 Declaration of Helsinki.

\section{Materials}

Stimuli for the greyscales task (see Nicholls et al., 1999) were administered on an IBM clone computer (PIV 2.6 GHz) interfaced with a 19" LCD monitor running at 1024 x 768 resolution. The maximum resolution of the monitor was $1400 \times 1050$ and the refresh rate was 8 
ms. A series of 320 rectangular grayscale pairs, of 5 different lengths: 110, 132, 154, 176 and 198 mm, were administered using E-prime (Psychology Software Tools, Inc., Pittsburgh, USA; www.pstnet.com/eprime). Length was varied to discourage the use of a standard response and to keep consistent with prior research employing this task (e.g., Nicholls et al., 1999; 2003;

Nicholls \& Roberts, 2002). Eighty of the greyscales were two mirror-reversed images where one appeared directly above the other, with one of the images being dark on the left side and the other dark on the right. The remaining 240 greyscales exhibited luminance differences with one image having either 250, 500, or 1000 more dark pixels than the other (see Orr \& Nicholls, 2005 who also used luminance differences). Greyscales were presented at $9.2^{\circ}$ visual angle either above (upper) or below (lower) the centre of the VF (see Appendix H). Participants viewed a fixation cross in the centre of the screen for one second prior to each trial. The stimulus was visible immediately following the presentation of the fixation cross and presentation was either brief $(150 \mathrm{msec})$ or prolonged (maximum of $10 \mathrm{sec}$ ). For brief presentations a grey response screen was visible immediately following the trial for a maximum of 10 seconds. There were 160 trials in each presentation time condition, with 80 presented to the lower VF and 80 presented to the upper VF. Trials were separated into blocks based on presentation time.

In prolonged trials participants were given up to 10 seconds to respond, allowing them to explore the images. Participants were instructed to fixate on the fixation cross in the centre of the VF between each trial. However, as participants were not aware of which VF each trial would be presented in, it is possible that both VFs were examined. Therefore, for prolonged trials visual projections would likely not be as segregated as during $150 \mathrm{msec}$ presentations. Inputs to the magnocellular and parvocellular pathways may not have been as isolated under prolonged conditions either. However, it is believed that participants primarily examined the stimuli as 
opposed to exploring the VF in which nothing was visible. As shown with cueing paradigms, attention is preferentially directed toward cued locations (see Posner \& Petersen, 1990 for review) and the appearance of the greyscales images should have acted in a similar manner to direct attention toward the VF where they were located.

Participants were asked to indicate whether the image on the top or the bottom of each pair appeared darker, or whether both images appeared to be the same. Responses were made using the number pad keys 8,5 , and 2, where 8 indicated the top image being chosen, 2 represented the bottom image, and 5 indicated the images appeared the same. Responses, as well as response times, were recorded. A response bias score was calculated by subtracting the number of leftward responses from the number of rightward responses, with a negative score indicating a leftward bias (Nicholls et al., 1999). Responses where participants indicated that the images appeared to be the same were considered separately and were not used in the calculation of bias scores.

Eye movements from the participants' left eye were monitored using an SMI-REDII iView infrared monitoring system, with data collection following a nine-point calibration procedure for each test session. Separate computers, used to operate the eye-tracking system and stimulus administration program, were interfaced with a parallel port connection to ensure accurate timing of stimulus presentation and data collection. A chin rest was used to minimize participant movement. Although the majority of the participants were right-handed, and likely also right-eye dominant (Bourassa, McManus, \& Bryden, 1996), data were collected from the left eye as the calibration of the left eye was more accurate with the system we used (see Rezec \& Dobkins, 2004, who also used the left eye). Eye movements were recorded during all trials; however eye movement data from brief presentations were not meaningful. This was done to 
ensure that the procedure was the same for all trials and, as such, participants were not aware that eye movements were not usable during the brief presentations.

ILAB (Gitelman, 2002) for Matlab was used to analyze eye movement data. The total number of pixels examined in each quadrant [upper left (UL), lower left (LL), upper right (UR), lower right (LR)] was determined in order to see which areas of the VF participants were looking at most often during each trial. Although no conditions consisted of uniquely right or left VF presentations, comparisons were also made of overall eye movements to the left and right VFs, as well as the upper and lower VFs throughout the task. This was done to examine whether participants directed more eye movements toward the left VF when examining the stimuli. Data were subjected to a repeated-measures analysis of variance, with independent variables of: VF location of the trial (upper, lower) and VF quadrant (UL, LL, UR, LR).

\section{Procedure}

Following informed consent (see Appendix M), participants completed a demographic questionnaire addressing items such as sex, age, visual or hearing impairments, and handedness and footedness (see Appendix A; Elias et al., 1998). Following completion of the questionnaire, participants completed the greyscales task. Participants were asked to select the image that appeared to be darker and were informed at this time that some images would be visible for a maximum of ten seconds, whereas others would only be visible briefly. All participants were debriefed following participation (see Appendix R).

\section{Results}

\section{Behavioural Data}

A 2 x 2 repeated-measures ANOVA was computed with within-subjects independent variables of VF (upper, lower) and presentation time (prolonged, brief), with the dependent 
variable being bias score on the greyscales task. Although there was no main effect of VF, $F(1,42)=.281, p=.599$, there was a trend for a main effect of presentation time, $F(1,42)=3.145$, $p=.083$, indicating a slightly stronger leftward bias during brief presentation $(150 \mathrm{msec})$.

The interaction between VF and presentation time was significant, $F(1,42)=57.182$, $p<.001$ (see Figure 5-1). Post hoc paired-samples $t$-tests indicated that brief presentation led to a stronger leftward bias in the upper VF $(t(42)=5.523, p<.001)$, whereas prolonged presentation led to a stronger leftward bias in the lower VF $(t(42)=-2.963, p=.005)$. Leftward biases were significant in the lower VF during prolonged presentation, $t(42)=-4.797, p<.001$, and in the upper VF during brief presentation $t(42)=-4.662, p<.001$. There was a trend for a rightward bias in the upper VF during prolonged presentation, $t(42)=1.830, p=.074$; however no bias was seen in the lower VF during brief viewing, $t(42)=-.239, p=.812$. As six participants were left-handed and there has been some indication that handedness might influence perceptual asymmetries (Jewell \& McCourt, 2000), the repeated-measures ANOVA was repeated with handedness as a between subjects measure. There was no indication that handedness influenced direction of bias on the greyscales task $(F(1,41)=.529, p=.471)$ and the main effects and interaction remained unchanged by the inclusion of this variable. 


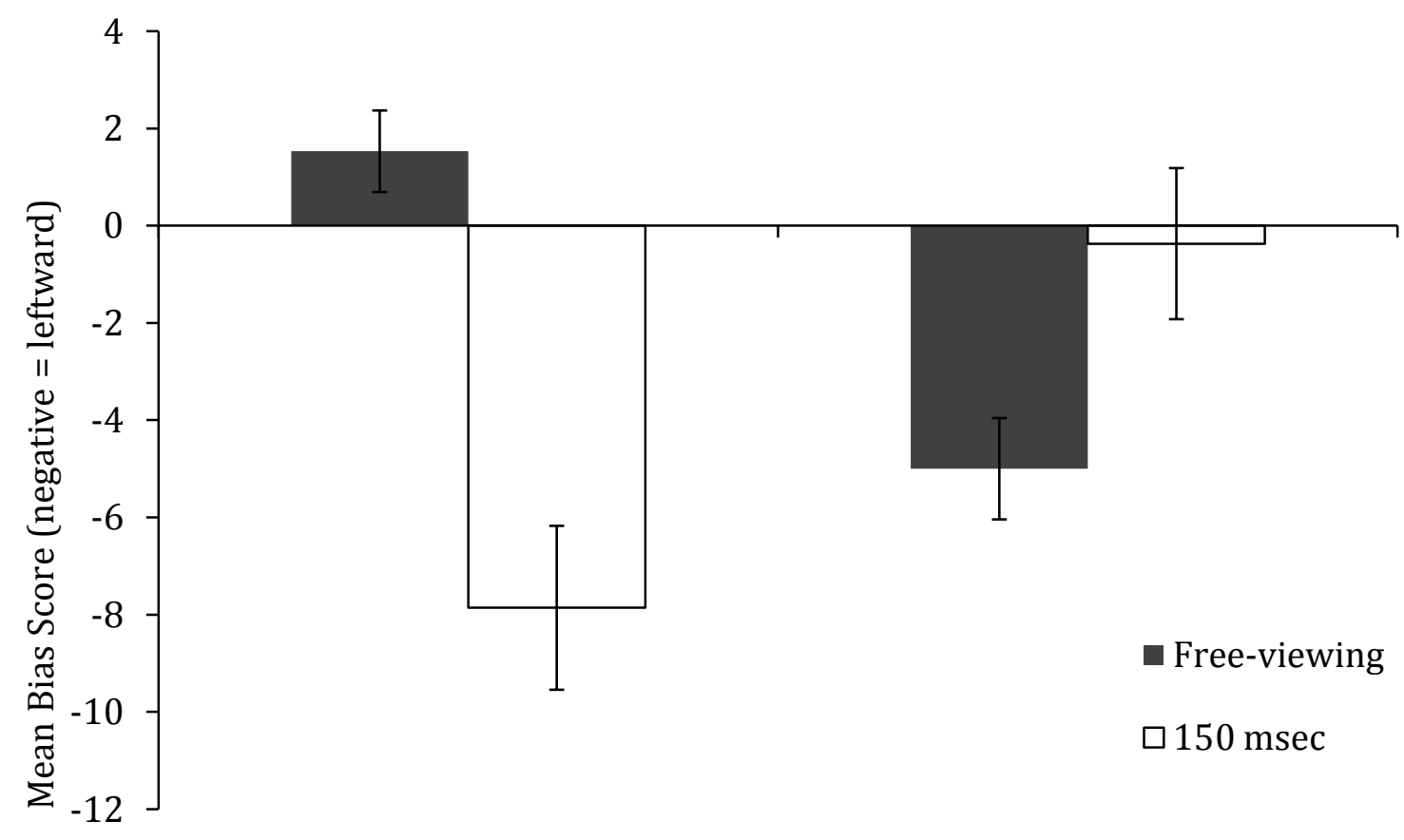

Upper Visual Field

Lower Visual Field

Figure 5-1. Interaction of presentation time and visual field. Error bars indicate the standard error of the mean.

Accuracy scores were calculated to show how accuracy was influenced by luminance difference (see Table 5-1). Recall that participants were given 3 response options in each instance, making chance performance 33 percent. Although the luminance differences used in the current study were larger than those employed previously, error rates were comparable to those observed in prior research using greyscale rectangle pairs with luminance differences (approximately 40 percent error rate: Nicholls et al., 2003; Nicholls et al., 2006b; Orr \& Nicholls, 2005). Examination of the accuracy scores illustrates that as luminance difference increased, participants were able to identify and select the image that was actually darker the majority of the time. Accuracy was higher and increased at a faster rate for prolonged trials than for brief trials (see Table 5-1), which is to be expected as participants were able to examine the images for a longer period of time during prolonged presentation. For comparisons between 
equiluminant presentations and the 1000 pixel difference condition, both brief and prolonged presentations showed a significant increase; however the mean increase was greater for prolonged presentation, $t(42)=-2.660, p=.011$.

Table 5-1. Accuracy scores based on luminance difference and comparison of percentage increase as pixel difference increased. Comparisons were made between the two closest pixel differences (equiluminant and 250; 250 and 500; 500 and 1000) and also for the overall increase in accuracy (equiluminant and 1000).

\begin{tabular}{llll}
\hline Luminance Condition & Presentation Time & Percent Correct & Comparison $p$ value \\
\hline Equiluminant & Prolonged & $42 \%$ & (to 250) $p=.506$ \\
& Brief & $28 \%$ & (to 250) $p=.008$ \\
250 pixel difference & Prolonged & $45 \%$ & (to 500) $p<.001$ \\
& Brief & $38 \%$ & (to 500) $p=.001$ \\
500 pixel difference & Prolonged & $60 \%$ & (to 1000) $p<.001$ \\
& Brief & $44 \%$ & (to 1000) $p=.667$ \\
1000 pixel difference & Prolonged & $69 \%$ & (to 0) $p<.001$ \\
& Brief & $45 \%$ & (to 0) $p<.001$ \\
\hline
\end{tabular}

\section{Eye Tracking Data}

Due to technical difficulty in recording eye tracking data from some individuals (i.e., wearing makeup or glasses), eye tracking data were only available for 31 participants. Although these participants were excluded from the eye tracking analyses, they were included in behavioural analyses. Both eye blinks and saccades were removed prior to analyses. A 2 x 4 repeated-measures analysis of variance was computed with VF where trial was presented (upper, lower) and VF quadrant examined (UL, LL, UR, LR) as within-subjects variables and total number of pixels examined as the dependent variable. The main effect of VF was not significant, $F(1,30)=.396, p=.534$, but there was an overall main effect of quadrant, $F(3,90)=6.726, p<.001$. Pairwise comparisons (using a Bonferroni correction) indicated the LL quadrant was examined significantly more often than all other quadrants (UL, $p=.008$; UR, $p=.019$; LR, $p=.002$ ).

There was also a significant VF by quadrant interaction $(F(3,90)=35.988, p<.001)$. Paired-samples $t$-tests were performed to examine which conditions differed from one another. 
For lower VF trials, significantly more pixels were examined in the LL quadrant than in the UL $(p<.001), \mathrm{UR}(p<.001)$, and LR $(p=.037)$ quadrants. Further, significantly more pixels were examined in the LR quadrant than in the UL $(p=.003)$ and UR $(p<.001)$ quadrants for lower VF trials. Therefore, participants spent more time examining the VF where stimuli were presented. When the greyscales were presented to the upper VF, more pixels were examined in the UL $(p=.001)$ and UR $(p<.001)$ quadrants than in the LR quadrant. This again illustrates that more time was spent examining the upper VF quadrants when stimuli were presented in the upper VF.

Paired-samples $t$-tests were used for overall comparisons of the left and right sides and of the upper and lower VFs. Significantly more pixels were examined in the left VF $(t(32)=2.153$, $p=.039)$ and the lower VF $(t(32)=-2.942, p=.006)$ during prolonged presentation.

\section{Discussion}

The strength of the leftward bias varied depending on VF presentation and was also modulated by length of presentation time. This is the first study to compare these factors using a within-subjects design. Participants demonstrated a stronger left bias in the upper VF during brief presentation and a stronger left bias in the lower VF during prolonged presentation. These results are in line with prior research in this area which has shown a stronger left bias in the upper VF when using $150 \mathrm{msec}$ presentations (McCourt \& Garlinghouse, 2000; McCourt \& Jewell, 1999) and a stronger left bias in the lower VF during prolonged presentation times (Barrett et al., 2000; Thomas \& Elias, 2010). In addition, eye tracking data illustrated that participants examined the left VF and the lower VF most often during prolonged presentations, consistent with a subsequent leftward response bias in the lower VF.

The leftward bias seen in the lower VF on prolonged trials stands in contrast to previously reported upward biases on line bisection (Bradshaw et al., 1985; Drain \& Reuter- 
Lorenz, 1996; McCourt \& Olafson, 1997) and greyscales (Nicholls et al., 2004; Nicholls et al., 2006b) tasks. However, these biases were observed for vertical stimuli as opposed to horizontal ones. It is difficult to compare these two manipulations as presenting stimuli to either the upper or the lower VF leads to different neural activation than stimuli that are presented in the centre of the VF. A trend for a rightward bias in the upper VF during prolonged presentation was also observed. This result can be interpreted in the context of the relation between the upper VF and the right side and the lower VF and left side (see Christman \& Niebauer, 1997 for a review).

There is considerable evidence showing that upper-lower differences are associated with left-right differences. Overall, tasks demonstrating an upper VF advantage have also shown a right VF advantage, whereas those showing a lower VF advantage demonstrate a left VF advantage (Christman \& Niebauer, 1997). The majority of results show that upper and right VF advantages are observed for local processing, categorical spatial judgments and in visual search. In contrast to this, lower and left VF advantages are seen for global processing, coordinate spatial judgments and global motion (Christman \& Niebauer, 1997).

The current finding of a slight rightward bias in the upper VF and a leftward bias in the lower VF are consistent with prior research showing a relation between the upper and right VFs, and the lower and left VFs. However, we only observed this relation in the prolonged condition. As the VF differences observed in the current study occurred on the same task, time given to examine the stimuli appears to underlie the upper and lower VF differences. Although prior work has not considered presentation time, it appears that saccadic eye movements as well as the opportunity to examine the stimuli more in depth could lead the left bias to be stronger in the lower VF. 
It is of interest to note that eye movements made toward upper VF targets are quicker than are those made to lower VF targets (e.g., Heywood \& Churcher, 1980; Honda \& Findlay, 1992). Eye movements would likely be minimal in the brief presentation condition; however it could be the case that upper VF targets were better viewed than lower VF targets in the brief condition. If eye movements were made in response to stimuli, upper VF targets would elicit quicker eye movements than would those in the lower VF, possibly making upper VF stimuli slightly more detectable than lower VF ones. Individuals also tend to elevate their eyes when attempting to scan information in memory (Kinsbourne, 1972). If this strategy were used in choosing which target appeared to be darker in the brief condition, it could explain the occurrence of a stronger left bias for upper VF presentations as well as the lack of bias in the lower VF during brief viewing.

As the leftward bias was observed under brief viewing conditions, scanning and reading habits (Chokron et al., 1998; Manning et al., 1990) are an unlikely explanation. As the task used in the current study required simple button press responses and keyboard responses evoke a minimal amount of motor movement (i.e., McCourt \& Olafson, 1997), pre-motor and motor biases do not provide an adequate explanation for the leftward bias either. Lateral asymmetries in cerebral activation are suggested to underlie the leftward bias (Schiff \& Truchon, 1993), as the right hemisphere is primarily responsible for spatial attention (Corbetta et al., 1995; Posner \& Petersen, 1990; Siman-Tov et al., 2007, but c.f. Giesbrecht, Kingstone, Handy, Hopfinger, \& Mangun, 2006). The current results appear to be consistent with a leftward perceptual/attentional bias, resulting from primarily right hemisphere activation; however this explanation does not account for why the strength of the left bias differs in the upper and lower VFs based on presentation time. 
It has been suggested that upper and lower VF differences are related to viewing distance as well as visual stream processing differences. The upper and lower VFs are thought to correspond with the processing of extrapersonal and peripersonal space, respectively (Previc, 1990). Stimuli that are located in the lower VF are more likely to be encountered, suggesting that they are also likely to be located in peripersonal space. Similarly, objects in the upper VF are more likely to be found in extrapersonal space, and will most often be brought into both the lower VF and peripersonal space for interaction. It is further suggested that the upper VF and extrapersonal space are mediated primarily by the ventral stream, whereas the lower VF and peripersonal space are processed predominantly by the dorsal stream (Previc, 1990).

Neuroimaging evidence partially supports this suggestion as Weiss et al. (2000) observed greater neural activity in the dorsal stream when processing information in peripersonal space and greater ventral stream activation for extrapersonal space processing. Previc, Breitmeyer, and Weinstein (1995) report evidence in support of the relation between the upper VF and extrapersonal space, and the lower VF and peripersonal space. An upper VF advantage for reaction time and accuracy was observed on a discrimination task using random dot stereograms when performed in extrapersonal space (Previc et al., 1995). The lower left quadrant showed the greatest impairment in discriminating targets in extrapersonal space, which the authors' conclude is consistent with Previc's (1990) suggestion that the lower VF is particularly important to peripersonal space tasks. A reaction time advantage has also been observed in extrapersonal space and upper VF conditions (Previc \& Blume, 1993). Previous research has typically shown a stronger leftward bias in peripersonal space than in extrapersonal space (Bjoertomt et al., 2002; Garza et al., 2008). Consistent with the relation between viewing distance and the upper and 
lower VFs, we observed a stronger leftward bias in the lower VF on a task completed in peripersonal space.

Vertical biases are thought to result from dorsal and ventral visual stream processing differences (Drain \& Reuter-Lorenz, 1996). Each visual stream is specialized for specific tasks, showing increased activation during these tasks; however processing differences between the two streams only indicate a relative advantage as the visual streams are not entirely isolated and have been shown to interact (Goodale \& Westwood, 2004). The dorsal stream, which is primarily (though not exclusively) associated with the magnocellular pathway, processes motion and also shows a temporal processing advantage due to its ability to respond quickly (Goodale $\&$ Milner, 1992; Livingstone \& Hubel, 1988; Nieuwenhuis et al., 2008). The ventral stream, which is an extension of the parvocellular pathway, processes colour and form and because of its slower response time, it allows for greater spatial segregation (Goodale \& Milner, 1992; Livingstone \& Hubel, 1988; Nieuwenhuis et al., 2008). Although the magnocellular and parvocellular pathways are specialised for specific functions, they are only partially segregated beyond the lateral geniculate nucleus and into the cortex (Nieuwenhuis et al., 2008).

According to Previc's (1990) theory of visual attention, a lower VF bias would result from primarily dorsal pathway activation. It has been suggested that dorsal and ventral stream processing differences might underlie vertical attention differences (Drain \& Reuter-Lorenz, 1996; Post, O`Malley, Yeh, \& Bethel, 2006). Drain and Reuter-Lorenz (1996) suggest that in the neurologically normal brain, the upward bias of the ventral pathway is stronger than the downward bias of the dorsal pathway. Further, tasks which are primarily carried out by the ventral pathway will show an accentuated upper VF bias and those that primarily activate the dorsal pathway will show a lower VF bias. The design of the current study limits the ability to 
discuss these results in relation to visual stream processing differences. Future research looking at visual stream processing differences more specifically as they relate to VF differences would extend the current findings as they relate to this potential explanation.

Evidence in support of an interaction between horizontal and vertical neglect whereby neglect is most severe in the lower left VF (Rubens, 1985) further suggests that visual stream processing differences provide a potential explanation for VF differences. Hemispatial neglect patients can exhibit either upward or downward neglect. Patients with occipitoparietal lesions demonstrate neglect of the lower VF (Butter, Evans, Kirsch, \& Kewman, 1989; Rapcsak, Cimino, \& Heilman, 1988), whereas occipitotemporal damage leads to upper VF neglect (Shelton, Bowers, \& Heilman, 1990). Drain and Reuter-Lorenz (1996) suggest that dorsal and ventral regions in each hemisphere may be mutually inhibitory in controlling vertical orienting. Parietal damage may decrease dorsal activation, leading to an upper VF bias as ventral stream activation is increased. In contrast, occipitotemporal damage may lead to increased dorsal activation, creating a lower VF bias as ventral stream activity is lessened. Therefore, it may be the case that dorsal and ventral stream processing differences underlie VF differences in perceptual asymmetries. Tasks that target dorsal and ventral stream processing would be useful in future studies to better segregate visual pathway projections and to allow this relationship to be investigated more directly.

The current results illustrate the importance of considering presentation time. When participants are given varying amounts of time to examine stimuli it significantly influences the strength of the left bias. Scanning and saccadic eye movements could lead the left bias to be stronger in the lower VF, whereas brief presentations, which do not afford lengthy examination of the stimuli, lead the left bias to be stronger in the upper VF. The precise mechanisms 
underlying these differences are unclear based on the current study; however it seems likely that visual stream processing differences could underlie VF differences.

As the upper and lower VFs are proposed to be linked to ventral and dorsal stream processing respectively (Previc, 1990), processing differences might lead to the VF differences observed in perceptual asymmetries. A more direct manipulation of dorsal and ventral processing advantages incorporating properties such as motion and colour could provide further support for this suggestion. VF differences should be considered when studying perceptual asymmetries as central presentations mask the effect of spatial location on the strength of the leftward bias. 


\section{CHAPTER 6 \\ DORSAL AND VENTRAL STREAM CONTRIBUTIONS TO PERCEPTUAL ASYMMETRIES}

\section{Introduction}

Neurologically normal individuals tend to pay more attention to the left side of space, which results in a leftward spatial bias. This left bias has been observed on several tasks, including line bisection (Luh, 1995; McCourt \& Jewell, 1999), the landmark task (Dufour et al., 2007) and the greyscales task (Nicholls et al., 1999; Thomas \& Elias, 2010). There are two types of line bisection tasks: manual line bisection (Luh, 1995), where participants use a stylus to bisect a line and perceptual line bisection (i.e., Dufour, et al., 2007; McCourt \& Jewell, 1999; Rolfe et al., 2008), where participants are presented with a pre-transected line and asked to make a judgment regarding the location of the transection mark. When bisecting manually (Luh, 1995), participants bisect to the left of centre and when asked to select whether the transector is located to the left or right of centre on computerized line bisection tasks (i.e., McCourt \& Jewell, 1999; Rolfe et al., 2008), participants typically indicate that the mark is to the right of centre, meaning the left side of the line is longer. When performing the landmark task, individuals tend to indicate that the left side of the line is longer the majority of the time (Dufour et al., 2007). This bias has been referred to as pseudoneglect (Bowers \& Heilman, 1980; Jewell \& McCourt, 2000) in the line bisection literature due to its similarity to clinical hemineglect (McCourt \& Jewell, 1999).

The greyscales task examines lateral biases in judgments of luminosity by having participants select the image that appears to be darker (or brighter) overall (Nicholls et al., 1999). Participants are shown a pair of mirror-reversed rectangles, with one image being dark on the left side and the other dark on the right, and each gradually becomes brighter on the opposing side. 
Although the images are equiluminant, participants tend to select the image that is dark on the left side as being darker overall. Similar leftward biases have been observed on tasks assessing judgments of numerosity, size (Nicholls et al., 1999), and distance (Krupp et al., 2010). On these tasks, participants typically choose the image with the salient feature (which appears to be larger, closer, etc...) on the left side as well (Krupp et al., 2010; Nicholls et al., 1999).

Interestingly, lateral biases differ in the upper and lower VFs. McCourt and Garlinghouse (2000) and McCourt and Jewell (1999) investigated VF differences using computerized line bisection. Pre-transected lines were presented tachistoscopically $(150 \mathrm{msec})$ at $3.6^{\circ}$ and $5.8^{\circ}$ visual angle, respectively, either above or below the midline. Participants indicated the location of the transection mark with respect to the perceived midpoint of the line. In both instances, McCourt and colleagues $(1999 ; 2000)$ found a stronger leftward bias when lines were located in the upper compared to the lower VF. Using a free-viewing manual line bisection task, Barrett et al. (2000) investigated upper and lower VF differences. Participants were given an unlimited amount of time to perform bisections at $40.5 \mathrm{~cm}$ either above or below the horizontal plane. Contrary to the findings of McCourt and colleagues, Barrett et al. (2000) found a stronger left bias in the lower VF.

Upper and lower VF differences are thought to relate to space-based and visual stream processing differences. The upper VF, extrapersonal space and ventral stream processing are thought to be related, as are the lower VF, peripersonal space and dorsal stream processing (Previc, 1990). From an evolutionary perspective, stimuli in the upper VF are more likely to be located at a distance in extrapersonal space (Previc, 1990). Likewise, stimuli in the lower VF are more likely to be located in peripersonal space. As stimuli in extrapersonal space are brought into peripersonal space, they are typically also brought into the lower VF for interaction (Previc, 
1990). Evidence in support of this relation has been demonstrated as an upper VF advantage for reaction time and accuracy was observed on a random dot stereogram discrimination task (Previc et al., 1995) and for reaction time on visual search (Previc \& Blume, 1993) when performed in extrapersonal space. The lower left quadrant showed the greatest impairment in discriminating targets in extrapersonal space, suggesting the lower VF is particularly important to peripersonal space tasks (Previc et al., 1995). Weiss et al. (2000) show support for the relation between peripersonal space activity and dorsal stream activation as well as ventral stream activation and the processing of information in extrapersonal space.

Thomas and Elias (2010) used the greyscales task to examine the interaction of VF and space-based differences. They presented the greyscales task at $9.2^{\circ}$ visual angle either above or below the midline in both peripersonal and extrapersonal space. Visual angle was maintained across conditions in order to maintain the size of the retinal image (Wilkinson \& Halligan, 2003). A stronger leftward bias was found in the lower VF for both viewing distances, suggesting space-based processing differences do not underlie VF differences. As the relation between viewing distance, $\mathrm{VF}$ and visual stream differences has not been studied extensively, it remains possible that visual stream processing differences relate to VF differences.

The dorsal and ventral visual streams are specialized for certain tasks and show increased activation during the completion of these tasks. These processing differences simply indicate a relative advantage as the visual streams interact with one another (Goodale \& Westwood, 2004) and are only partially segregated beyond the lateral geniculate nucleus and into the cortex (Nieuwenhuis et al., 2008). The ventral stream has a slower response time, allowing for greater spatial segregation and is preferential in the analysis of colour and form (Goodale \& Milner, 1992; Livingstone \& Hubel, 1988; Nieuwenhuis et al., 2008). The dorsal stream responds 
quickly, which allows for better temporal segregation, and also shows an advantage in processing motion (Goodale \& Milner, 1992; Livingstone \& Hubel, 1988; Nieuwenhuis et al., 2008). In addition the ventral visual stream is primarily a continuation of the parvocellular pathway and the dorsal stream is primarily an extension of the magnocellular pathway (Livingstone \& Hubel, 1988). This relationship suggests that tasks primarily targeting magnocellular and dorsal stream functioning (e.g., motion) or parvocellular and ventral stream processing (e.g., colour) provide a useful way to examine VF differences.

Several explanations have been put forth to explain the occurrence of left spatial biases, including scanning and reading habits (Chokron et al., 1998; Manning et al., 1990) or intentional/pre-motor biases (Brodie \& Pettigrew, 1996; Grimshaw \& Keillor, 2000; Heilman \& Valenstein, 1979). Nicholls and Roberts (2002) showed that these explanations were not sufficient as individuals with opposite reading patterns and bimanual responding both showed leftward biases. An attentional bias appears to be the most likely explanation as the right hemisphere, particularly the posterior parietal region, is primarily responsible for spatial attention (Corbetta et al., 1995; Niemeier et al., 2008; Posner \& Petersen, 1990; Posner \& Rothbart, 2007). This leads attention to be directed to the left side of space preferentially, a suggestion that is supported by neuroimaging data showing visuospatial attention networks are more activated by information in the left VF (Siman-Tov et al., 2007). Neuroimaging data also indicate that the right hemisphere is more active during line bisection and landmark tasks (Çiçek et al., 2009; Fink et al., 2000; Fink et al., 2001; Foxe et al., 2003).

The current study investigated potential dorsal and ventral stream processing differences in perceptual asymmetries using two exploratory measures. Modified greyscales tasks, incorporating either motion or isoluminant colour, were designed to target dorsal and ventral 
stream processing, respectively. Red and green isoluminant stimuli were used in the colour task (see Appendix S) and if ventral stream activity is responsible for the occurrence of the left bias, the image with the salient characteristic (i.e., which appears to be more green) on the left side should be selected the majority of the time. The motion task consisted of rectangles with either a black or a white background and pixels of the opposing colour moving almost uniformly across the rectangle. The direction of motion was eastbound in one rectangle and west in the other. If dorsal stream processing underlies perceptual asymmetries, the image with the salient feature (i.e., which appears to be darker) on the left side should be chosen the majority of the time.

The greyscales task was also administered as all other tasks were exploratory and it was desirable to ensure that the current sample was consistent with prior samples. A leftward bias was expected for both conditions. It was hypothesized that the dorsal stream would be preferential for the emergence of perceptual asymmetries, and that the task incorporating motion would lead to the strongest leftward bias, whereas the isoluminant colour task would not exhibit a strong bias. A dorsal processing advantage would be consistent with the previously observed stronger left bias in the lower VF on the greyscales task (Thomas \& Elias, 2010). In light of recent evidence showing that colour information is minimally accessible to guided reaching (White, Kerzel, \& Gegenfurtner, 2006), which is a dorsal task, the colour task might will also show a left bias; however the bias should be weaker than that observed with the motion task.

\section{Study 1}

\section{Method}

Participants. Eighty-nine undergraduate Psychology students (25 males; mean age $=20.26, S D=4.94)$ at the University of Saskatchewan participated in the current study. Students received course credit in exchange for participation. Based upon self-report (see 
Appendix A; Elias et al., 1998), eight participants were left-handed and all participants had normal or corrected-to-normal vision. This study was conducted with the ethical approval of the Behavioural Research Ethics Board at the University of Saskatchewan and has therefore been performed in accordance with the ethical standards laid down in the 1964 Declaration of Helsinki.

Materials. All tasks were administered on an IBM clone computer (PIV 2.6 GHz) interfaced with a 19" LCD monitor running at 1024 x 768 resolution. The maximum resolution of the monitor was $1400 \times 1050$ and the refresh rate was $8 \mathrm{~ms}$. Participants used a chin rest to minimize head movement and distance to the screen was $71.1 \mathrm{~cm}$. Stimulus pairs were presented in the centre of the VF, one directly above the other. All presentations were free-viewing and visible for a maximum of five seconds. Participants were asked to select whether the image on the top or the bottom of the pair appeared to include the salient characteristic. Responses were made using the number pad keys 8 and 2, with 8 representing the top image being chosen and 2 representing the bottom image. As simple button press and keyboard responses evoke a minimal amount of motor movement (i.e., McCourt \& Olafson, 1997), responses were not confounded by pre-motor or motor biases. A central fixation was presented prior to each trial and participants were free to scan the images prior to responding. Responses, as well as response times, were recorded. A response bias score was calculated by subtracting the number of leftward responses from the number of rightward responses and then dividing by the total number of trials, with a negative score indicating a leftward bias (Nicholls et al., 1999).

Greyscales Task. E-prime (Psychology Software Tools, Inc., Pittsburgh, USA; www.pstnet.com/eprime) was used to administer a series of rectangle pairs, of 4 different lengths: 132, 154, 176 and $198 \mathrm{~mm}$. For luminance tasks, rectangle pairs consisted of mirror- 
reversed pairs of rectangles going from black on one end to white on the other. One of the images was dark on the left side and the other was dark on the right. Stimuli from the greyscales task (see Appendix D; Nicholls et al., 1999) were used and participants were asked to identify which image appeared to be more black or more white, as opposed to which appeared darker or brighter. This was done to be consistent with the instructions for the other tasks that were administered in the current experiment. There were 64 trials in each condition (black, white).

Isoluminant Colour Tasks. Stimuli from the greyscales task were modified to be isoluminant red and green in colour as opposed to black and white. This was done by matching red and green colour values using the luminance feature in Paint Shop Pro 7.0 (Jasc Software). A Tektronix J6523-2 $1^{\circ}$ narrow angle luminance probe was then used to match the colours for isoluminance. Stimuli were visibly similar to the greyscales images (see Appendix S), and transitioned from red on one side to green on the other. In each pair one image was red on the left side and the other was red on the right. There were 64 trials in each condition (red, green), for a total of 128 trials. E-prime was also used to administer the isoluminant colour tasks.

Motion Tasks. The motion task was created using images that resembled those used in the greyscales task. Stimulus pairs consisted of two rectangles with a uniformly coloured background (black or white) and pixels of the opposing colour moved across the rectangle. The moving pixels travelled from east to west in one rectangle and from west to east in the other, making one rectangle either dark or bright on the left and the other dark or bright on the right. Motion was not entirely uniform as some rogue pixels travelled more randomly across the rectangle. This was done to avoid having participants follow the motion of any one particular pixel. All participants completed both conditions: which appeared to be darker for white 
rectangles with black pixels and which appeared to be brighter for black rectangles with white pixels. There were 128 trials in each condition, for a total of 256 motion trials.

Procedure. Following informed consent (see Appendix T), participants completed a demographic questionnaire addressing items such as sex, age, visual or hearing impairments, and handedness and footedness (see Appendix A; Elias et al., 1998). Following completion of the questionnaire, participants completed the greyscales, isoluminant colour and motion tasks. The order of presentation of the tasks was counterbalanced between participants, but opposing static conditions (i.e., more red and more green) were not presented consecutively to avoid carry over effects between tasks. Both motion conditions were presented consecutively as one task as a result of the program that was created to administer this task. All participants were debriefed following participation (see Appendix U).

\section{Results}

A $2 \times 2 \times 6$ repeated-measures ANOVA was computed using the within-subjects independent variable of condition (black, white, red, green, dark motion, bright motion) and between-subjects independent variables of handedness (right-handed, left-handed) and sex (male, female) and the dependent variable was the bias score on each task. As handedness did not exhibit a significant effect $(F(1,85)=.003, p=.958, M S E=.002)$, the analysis of variance was repeated without this variable. When handedness was not included, sex did not exhibit a significant effect, $F(1,87)=3.109, p=.081, M S E=1.944$, and it was therefore removed from the analysis. There was a significant main effect of condition, $F(5,440)=4.162, p=.001, M S E=2.531$. Pairwise comparisons of the main effect (with a Bonferroni correction) indicated significant differences between the black and green $(p=.002)$ and the red and green $(p=.020)$ conditions. 
Paired-samples $t$-tests were used to compare biases in opposing conditions (white and black, dark and bright motion, red and green) as no differences were expected between conditions (Bonferroni corrected $p$ value $=.017$ ). There were no significant differences between the black and white conditions $(t(88)=-1.442, p=.153)$ or the dark and bright motion conditions $(t(88)=2.282, p=.025)$ (see Table 6-1 for means and standard deviations). As there were no significant differences across these conditions, bias scores were created for luminance and motion by averaging scores from the dark and bright conditions. Interestingly, the red and green conditions were significantly different from one another, $(t(88)=-3.310, p=.001$; see Table 6-1), indicating that these conditions could not be considered together and were therefore kept separate for the remaining analyses.

Table 6-1. Means and standard deviations for all conditions in Study 1

\begin{tabular}{llc}
\hline Condition & Mean & Standard Deviation \\
\hline Black & -.265 & .492 \\
White & -.195 & .464 \\
Green & -.071 & .481 \\
Red & -.245 & .460 \\
Dark Motion & -.125 & .465 \\
Bright Motion & -.228 & .362 \\
Luminance (black + white) & -.230 & .420 \\
Motion (dark + bright) & -.232 & .372 \\
\hline
\end{tabular}

One-sample $t$-tests were used to determine whether significant biases were seen in each condition (Bonferroni corrected $p$ value $=.013$ ). Significant left biases were seen for luminance, $t(88)=-5.163, p<.001$, motion, $t(88)=-5.902, p<.001$, and red, $t(88)=-5.026, p<.001$. The bias on the green condition failed to reach significance, $t(88)=-5.026, p=.168$ (see Figure 6-1).

As it was suggested that the isoluminant colour task could show a bias, but that it would be significantly weaker than the biases seen in the other tasks, paired-samples $t$-tests were used to determine whether the strength of the left bias differed between conditions. A significant difference emerged between green and luminance, $t(88)=3.539, p=.001$, as well as between 
green and motion, $t(88)=3.065, p=.003$. Both luminance and motion exhibited stronger left biases than the green condition. No difference was seen between the luminance and motion conditions $(t(88)=.079, p=.937)$.

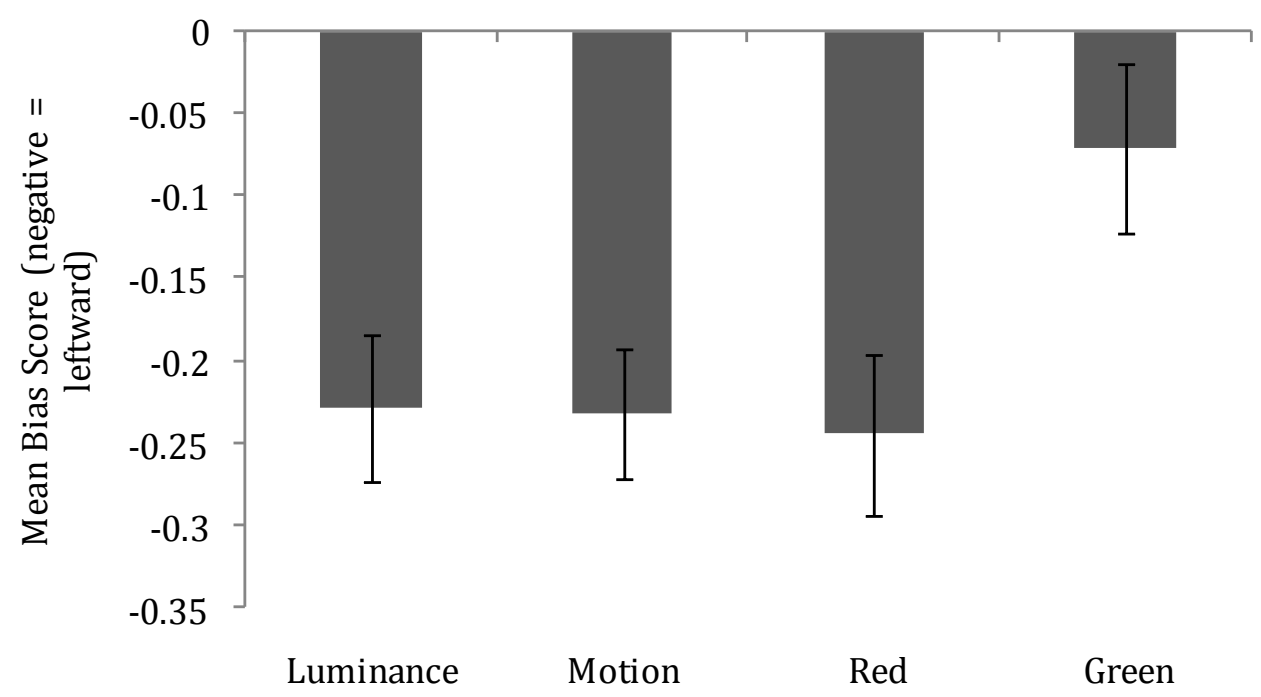

Figure 6-1. Mean bias scores for motion, luminance, red and green tasks completed in Experiment 1 of Study 5. Error bars indicate the standard error of the mean.

\section{Discussion}

As hypothesized, a leftward bias emerged for luminosity judgments, consistent with prior research using the greyscales task (Nicholls et al., 1999). This bias was seen despite the usage of slightly different instructions for the completion of the task. As expected, there was also a significant leftward bias on the motion task, indicating that the magnocellular pathway and dorsal stream processing are preferential to the emergence of perceptual asymmetries. However, an unexpected leftward bias was seen when making red judgments, but not green judgments. Biases on the motion and luminance tasks were significantly stronger than the bias seen on the more green judgments. This suggests that the parvocellular pathway and ventral stream processing does not allow the leftward bias to persist to the same extent as does dorsal stream processing. 
There is a great deal of evidence indicating that red captures attention more readily than other colours do (Bellizzi \& Hite, 1992; Clynes, 1977; Elliot \& Maier, 2007; Elliot, Maier, Moller, Friedman, \& Meinhardt, 2007; Lu \& Zhou, 2005; Maier, Barchfeld, Elliot, \& Pekrun, 2009; Mehta \& Zhu, 2009; Tchernikov \& Fallah, 2010; Wilson, 1966). If the colour red preferentially attracts attention, a leftward spatial bias might emerge simply as a result of the initial capture of attention by red, possibly toward the left side. Although this would lead to a response bias, it would not indicate that the parvocellular pathway was responsible for this bias. As a strong bias was seen on the motion task and no bias was seen for more green judgments, it would appear that isoluminant colour does not lead to a left bias; simply that red preferentially draws attention. The first study was repeated in order to determine whether the bias seen for red judgments would emerge a second time or whether the result was nebulous.

\section{Study 2}

In the first study the motion task exhibited a significant bias when participants were asked to choose either which was brighter or which was darker, indicating that the leftward bias persists on a task incorporating motion and targeting the magnocellular pathway and likely projecting to the dorsal stream. The dorsal stream therefore appears to be preferential to the emergence of perceptual asymmetries. There was no bias seen when choosing which image appeared to be more green on the isoluminant colour task, but a strong left bias was seen for red judgments. Therefore the red-green isoluminant colour task was repeated. As mentioned previously colour information is minimally accessible during tasks that elicit dorsal stream activation (White et al., 2006), and the colour task might also exhibit a slight left bias; however the bias should not be as strong as that observed on the greyscales or motion tasks. 
As the motion task exhibited the expected leftward bias, it is suggested that dorsal stream processing is preferential to the emergence of the left bias. This task was repeated to ensure that the bias would emerge again. Two presentation times were included for the motion task: prolonged ( 5 second maximum), and brief $(150 \mathrm{msec})$ to determine whether the bias could be maintained under brief viewing conditions. As prior research employing brief presentations (i.e., McCourt \& Garlinghouse, 2000; McCourt \& Jewell, 1999) has shown significant left biases, it was expected that the leftward bias on the motion task would persist on the $150 \mathrm{msec}$ condition.

\section{Method}

Participants. Forty-six undergraduate Psychology students (12 males; mean age=20.07, $S D=4.64)$ at the University of Saskatchewan participated in the current study. Students received course credit in exchange for participation. Based upon self-report (see Appendix A; Elias et al., 1998), two participants were left-handed and all participants had normal or corrected-to-normal vision. This study was conducted with the ethical approval of the Behavioural Research Ethics Board at the University of Saskatchewan and has therefore been performed in accordance with the ethical standards laid down in the 1964 Declaration of Helsinki.

Materials. All tasks were administered on an IBM clone computer (PIV 2.6 GHz) interfaced with a 19" LCD monitor running at 1024 x 768 resolution. The maximum resolution of the monitor was $1400 \times 1050$ and the refresh rate was $8 \mathrm{~ms}$. Participants used a chin rest to minimize head movement and distance to the screen was $71.1 \mathrm{~cm}$. Stimulus pairs were presented in the centre of the VF, one directly above the other. Participants were asked to select whether the image on the top or the bottom of the pair appeared to include the salient characteristic. Responses were made using the number pad keys 8 and 2, with 8 representing the top image being chosen and 2 representing the bottom image. A central fixation was presented prior to each 
trial and participants were free to scan the images prior to responding. Responses, as well as response times, were recorded. A response bias score was calculated by subtracting the number of leftward responses from the number of rightward responses and then dividing by the total number of trials, with a negative score indicating a leftward bias (Nicholls et al., 1999).

Isoluminant Colour Tasks. E-prime was used to administer the same red-green stimuli as were used in the first experiment (see Appendix S). All presentations were free-viewing and visible for a maximum of five seconds. All participants completed both tasks, with a total of 64 trials in each condition (red, green), leading to 128 trials.

Motion Tasks. The motion task from experiment one was also used in experiment two. Participants again completed both conditions: which appeared to be darker for white rectangles with black pixels and which appeared to be brighter for black rectangles with white pixels. There were two presentation time conditions in Study 2: prolonged and brief. During prolonged presentations participants were given a maximum of five seconds to respond. Brief presentation consisted of a $150 \mathrm{msec}$ presentation of the stimulus pair, followed by a five-second maximum response period. There were 128 trials in each condition, for a total of 256 motion trials in each presentation time condition (512 total motion trials).

Procedure. Following informed consent (see Appendix T), participants completed a demographic questionnaire addressing items such as sex, age, visual or hearing impairments, and handedness and footedness (see Appendix A; Elias et al., 1998). Following completion of the questionnaire, participants completed the tasks. The order of presentation of the tasks was counterbalanced between participants, but opposing static conditions (i.e., more red and more green) were not presented consecutively to avoid carry over effects between tasks. Both motion conditions were presented consecutively as one task as a result of the program that was created to 
administer this task but presentation time conditions were presented separately. All participants were debriefed following participation (see Appendix U).

\section{Results}

Data were unavailable for two participants in the brief motion condition due to a technical difficulty in recording the data. A $2 \times 2 \times 6$ repeated-measures ANOVA was computed using the within-subjects independent variable of condition (red, green, prolonged dark motion, prolonged bright motion, brief dark motion, brief bright motion) and between-subjects independent variables of handedness (right-handed, left-handed) and sex (male, female) and the dependent variable was the bias score on each task. As neither $\operatorname{sex}(F(1,38)=.271, p=.606$, $M S E=.182)$ nor handedness $(F(1,38)=.265, p=.609, M S E=.179)$ exhibited a significant effect, the ANOVA was repeated without these factors. There was a main effect of condition, $F(5,200)=3.235, p=.008, M S E=.259$. Pairwise comparisons of the main effect (with a Bonferroni correction) indicated significant differences between the brief dark motion condition and the green condition $(p=.018)$ and between the green and red conditions $(p=.003)$.

Paired-samples $t$-tests were used to compare biases in opposing conditions (dark and light motion, brief dark and light motion, red and green) as a significant difference was seen between red and green in Study 1. There were no significant differences between the dark and bright motion conditions for either prolonged, $t(45)=-1.548, p=.129$, or brief presentations, $t(43)=-$ 1.640, $p=.108$ (see Table 6-2). As there were no significant differences across these conditions, bias scores were created for prolonged and brief motion by averaging scores from the dark and bright conditions. Once again, the red and green conditions were significantly different, $(t(45)=-$ 3.838, $p<.001$; see Table 6-2), which suggests that the result obtained in Study 1 was likely not inaccurate. Red and green were considered separately for the remaining analyses. 
Table 6-2. Means and standard deviations for all conditions in Study 2

\begin{tabular}{llc}
\hline Condition & Mean & Standard Deviation \\
\hline Green & -.169 & .425 \\
Red & -.331 & .414 \\
Prolonged Dark Motion & -.314 & .418 \\
Prolonged Bright Motion & -.215 & .387 \\
Brief Dark Motion & -.367 & .421 \\
Brief Bright Motion & -.288 & .409 \\
Prolonged Motion (dark + bright) & -.265 & .343 \\
Brief Motion (dark + bright) & -.327 & .383 \\
\hline
\end{tabular}

One-sample $t$-tests were used to determine whether significant biases were seen in each condition (Bonferroni corrected $p$ value =.013). Significant leftward biases were seen for prolonged motion $(t(45)=-5.067, p<.001)$, brief motion $(t(45)=-5.671, p<.001)$, and red $(t(45)=-$ $5.431, p<.001)$ conditions. The bias on the green condition just reached significance $(t(45)=-$ 2.703, $p=.010$; see Figure 6-2).

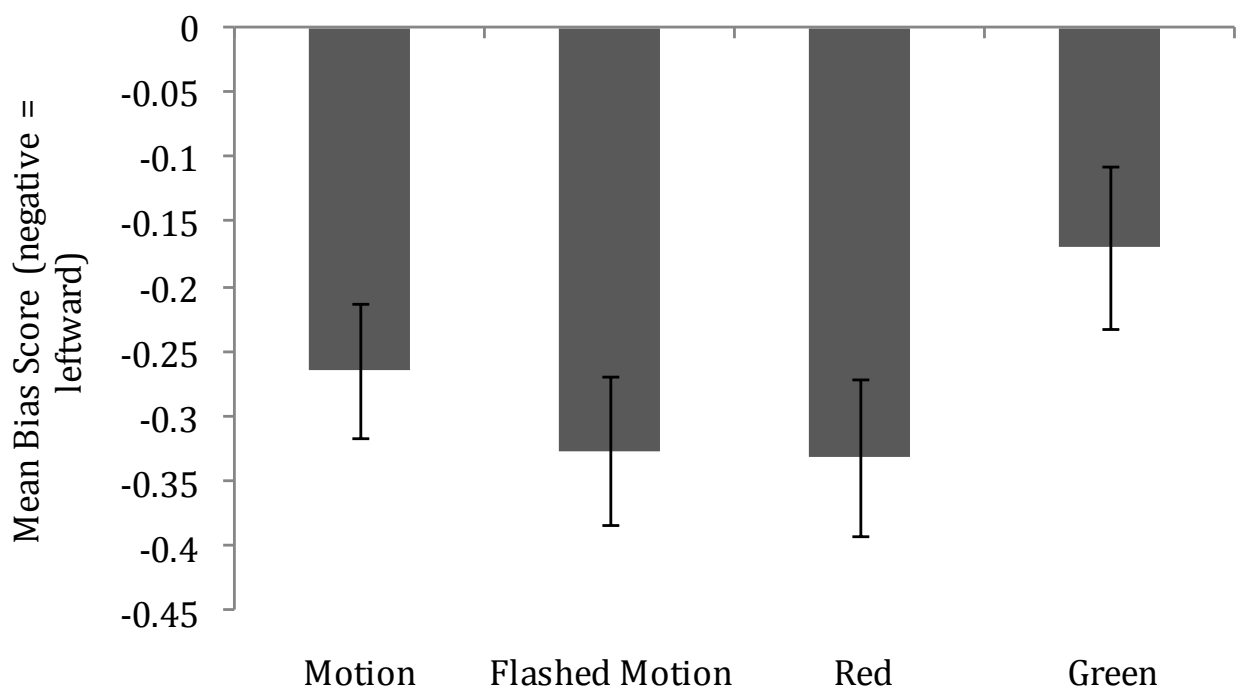

Figure 6-2. Mean bias scores for free-viewing and non-free-viewing motion as well as red and green tasks completed in Experiment 2 of Study 5. Error bars indicate the standard error of the mean.

As it was suggested that the isoluminant colour task could show a bias, but that it would be significantly weaker than the biases seen in the other tasks, paired-samples $t$-tests were used to determine whether the strength of the left bias differed between conditions. A significant 
difference emerged between green and brief motion, $t(43)=2.786, p=.008$, as well as between green and prolonged motion, $t(42)=2.243, p=.030$. Both prolonged and brief motion exhibited stronger left biases than the green condition. No difference was seen between the prolonged and brief motion conditions $(t(40)=.669, p=.507)$.

\section{Discussion}

In order to avoid the potential confound created by using red-green stimuli on the isoluminant colour task, blue-yellow isoluminant stimuli were used instead.

\section{Study 3}

A significant leftward bias emerged on the motion task in both prior studies. Further, the strength of the leftward spatial bias during the motion task can be seen as it persisted during 150 msec presentation. These results suggest that the left bias is likely attributable to dorsal stream processing. As in the first study, there was no bias observed on the green judgments in the isoluminant colour task; however a significant leftward bias was seen for red judgments, further suggesting that red captures attention differently than green does. The current findings, along with prior research suggesting red might preferentially capture attention (Bellizzi \& Hite, 1992; Clynes, 1977; Elliot \& Maier, 2007; Elliot et al., 2007; Lu \& Zhou, 2005; Maier et al., 2009; Mehta \& Zhu, 2009; Tchernikov \& Fallah, 2010; Wilson, 1966), suggest that it not appropriate to use red-green stimuli on the isoluminant colour task. Instead, blue-yellow isoluminant stimuli were used in the third study to better examine whether a bias persists with isoluminant colour. As mentioned previously colour information is minimally accessible during tasks that elicit dorsal stream activation (White et al., 2006), and the colour task might also exhibit a slight left bias; however the bias should not be as strong as that observed on the greyscales task. 
A second manipulation was made to the red-green isoluminant colour task to determine whether the significant leftward bias on the red judgments could be eliminated when a $150 \mathrm{msec}$ presentation was used. No bias was expected for brief presentations of the red-green isoluminant task for either red or green judgments. This would suggest that red is able to capture attention when lengthier presentations are used, but when processing time is decreased the bias is absent. This would provide evidence in support of visual stream processing differences underlying VF differences as parvocellular processing eliminates the left bias. If the dorsal stream is a strong contributor to the leftward spatial bias then the bias should not occur when $150 \mathrm{msec}$ presentations were used with the colour task, even if red captures attention more readily.

\section{Method}

Participants. Forty undergraduate Psychology students ( 8 males; mean age $=20.15$, $S D=3.74$ ) at the University of Saskatchewan participated in the current study. Students received course credit in exchange for participation. Based upon self-report (see Appendix A; Elias et al., 1998), four participants were left-handed and all participants had normal or corrected-to-normal vision. This study was conducted with the ethical approval of the Behavioural Research Ethics Board at the University of Saskatchewan and has therefore been performed in accordance with the ethical standards laid down in the 1964 Declaration of Helsinki.

Materials. All tasks were administered on an IBM clone computer (PIV 2.6 GHz) interfaced with a 19" LCD monitor running at 1024 x 768 resolution. The maximum resolution of the monitor was $1400 \times 1050$ and the refresh rate was $8 \mathrm{~ms}$. Participants used a chin rest to minimize head movement and distance to the screen was $71.1 \mathrm{~cm}$. Stimulus pairs were presented in the centre of the VF, one directly above the other. Participants were asked to select whether the image on the top or the bottom of the pair appeared to include the salient characteristic. 
Responses were made using the number pad keys 8 and 2, with 8 representing the top image being chosen and 2 representing the bottom image. A central fixation was presented prior to each trial and participants were free to scan the images prior to responding. Responses, as well as response times, were recorded. A response bias score was calculated by subtracting the number of leftward responses from the number of rightward responses and then dividing by the total number of trials, with a negative score indicating a leftward bias (Nicholls et al., 1999).

Isoluminant Colour Tasks. E-prime was used to administer red-green stimuli from Study 1. Brief presentation consisted of a $150 \mathrm{msec}$ presentation of the stimulus pair, followed by a five-second maximum response period. Participants again completed both tasks, with a total of 64 trials in each condition (red, green).

Red and green stimuli from Studies 1 and 2 were modified using the luminance feature in Paint Shop Pro 7.0 (Jasc Software). The colour values for blue and yellow were matched to create blue-yellow isoluminant stimuli. A Tektronix J6523-2 $1^{\circ}$ narrow angle luminance probe was then used to match the colours for isoluminance. Stimuli were again visibly similar to the greyscales images (see Appendix V), and transitioned from blue on one side to yellow on the other. In each pair one image was blue on the left side and the other was blue on the right side. Presentations were free-viewing and visible for a maximum of five seconds. There were 64 trials in each condition (yellow, blue), making a total of 256 trials on the isoluminant colour tasks (128 prolonged and 128 brief).

Greyscales Task. E-prime was also used to administer a series of 36 greyscales, of 5 different lengths: 110, 132, 154, 176 and $198 \mathrm{~mm}$. Two mirror-reversed images were presented, with one of the images being dark on the left side and the other dark on the right. Participants were asked to select which image appeared to be darker (Nicholls et al., 1999). 
Procedure. Following informed consent (see Appendix T), participants completed a demographic questionnaire addressing items such as sex, age, visual or hearing impairments, and handedness and footedness (see Appendix A; Elias et al., 1998). Following completion of the questionnaire, participants completed the tasks. The order of presentation of the tasks was counterbalanced between participants, but opposing colour conditions (i.e., more red and more green) were not presented consecutively to avoid carry over effects between tasks. The greyscales task was always administered at the end of the protocol in order to avoid any priming effects that this task might have created as the other tasks were exploratory. All participants were debriefed following participation (see Appendix U).

\section{Results}

A $2 \times 2 \times 5$ repeated-measures ANOVA was computed using the within-subjects independent variable of condition (blue, yellow, brief red, brief green, greyscales) and betweensubjects independent variables of handedness (right-handed, left-handed) and sex (male, female) and the dependent variable was the bias score on each task. As neither sex $(F(1,36)=.109$, $p=.743, M S E=.065)$ nor handedness $(F(1,36)=1.111, p=.299, M S E=.657)$ exhibited a significant effect, the ANOVA was repeated without these factors. There was no main effect of condition, $F(4,156)=.801, p=.527, M S E=.056$.

Paired-samples $t$-tests were used to compare biases in opposing conditions (blue and yellow, brief red and green; Bonferroni corrected $p$ value $=.025$ ) as a significant difference was seen between red and green in Studies 1 and 2. There were no significant differences between brief red and green, $t(39)=.337, p=.738$, or blue and yellow, $t(39)=-2.082, p=.044$ (see Table 63). As there were no significant differences between conditions, bias scores were created for colour (blue-yellow) and brief colour (red-green) by averaging scores across conditions. 
Table 6-3. Means and standard deviations for all conditions in Study 3

\begin{tabular}{llc}
\hline Condition & Mean & Standard Deviation \\
\hline Blue & -.153 & .398 \\
Yellow & -.050 & .417 \\
Brief Red & -.092 & .405 \\
Brief Green & -.112 & .420 \\
Greyscales & -.115 & .434 \\
Colour (blue + yellow) & -.102 & .376 \\
Brief Colour (red + green) & -.102 & .367 \\
\hline
\end{tabular}

One-sample $t$-tests were used to determine whether significant biases were seen in each condition (Bonferroni corrected $p$ value $=.017$ ). No significant biases were seen for colour $(t(39)=-1.713, p=.095)$ or for brief colour $(t(39)=-1.759, p=.086$; see Table 6-3); however no significant bias was seen on the greyscales task either $(t(39)=-1.671, p=.103$; see Figure 6-3).

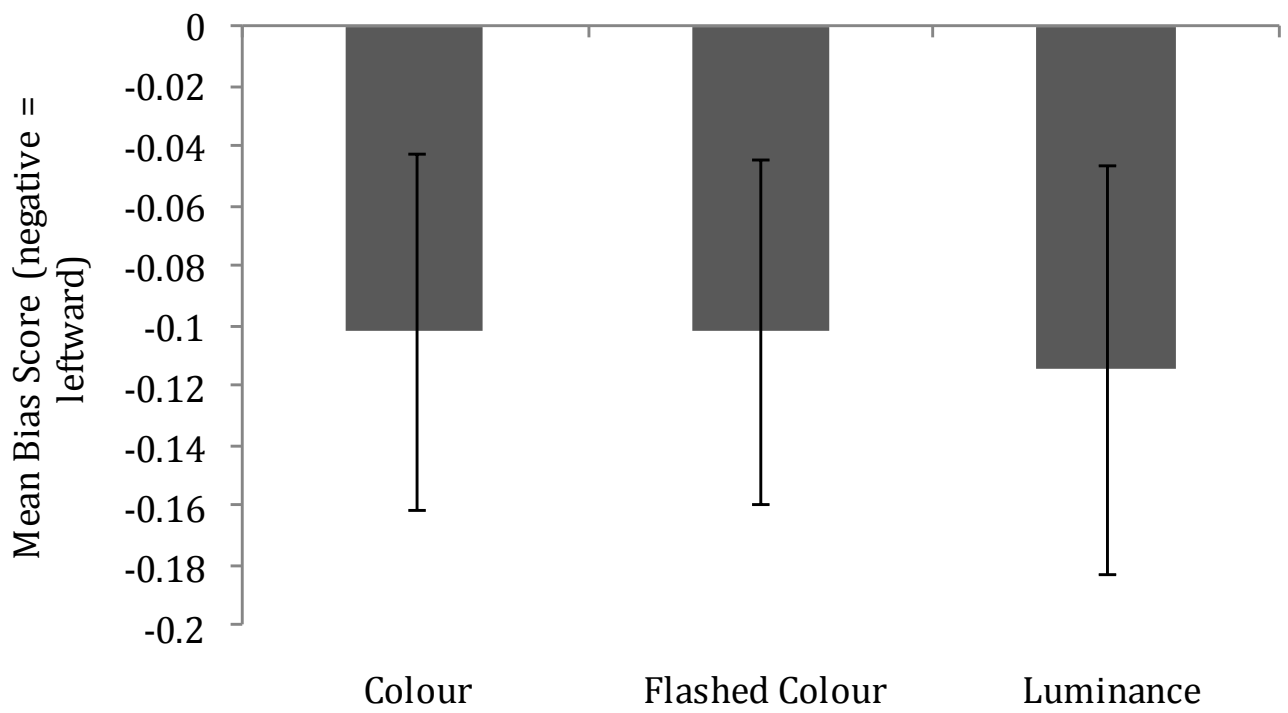

Figure 6-3. Mean bias scores for blue and yellow tasks, non-free-viewing green and red tasks and the greyscales task completed in Experiment 3 of Study 5. Error bars indicate the standard error of the mean.

As the result on the greyscales task was unexpected, bias scores on this task were examined more closely. An independent-samples $t$-test was used to examine the effect of sex (male, female) on bias scores on the greyscales task. There was a significant sex difference, $t(38)=2.263, p=.029$, with males showing a trend for a right spatial bias $(t(7)=2.074, p=.077$, $M=.181, S D=.246)$ and females showing a significant left spatial bias $(t(31)=-2.414, p=.022, M=$ 
$-.188, S D=.441)$. Due to the small number of males who were included in the sample $(\mathrm{n}=8)$, it is not possible to make any generalizations regarding this sex difference; however it does explain the lack of a significant bias observed on the greyscales task in the current study.

Analysis from Studies 1, 2 and 3. Data from the three studies were combined to compare luminance, motion and colour (combining blue, yellow and green data). One-sample $t$ tests were used to examine whether significant biases were seen in each condition. Significant biases were seen for luminance, $t(128)=-5.175, p<.001$, motion, $t(131)=-7.723, p<.001$, and colour, $t(174)=-3.095, p=.002$, conditions (see Figure 6-4).

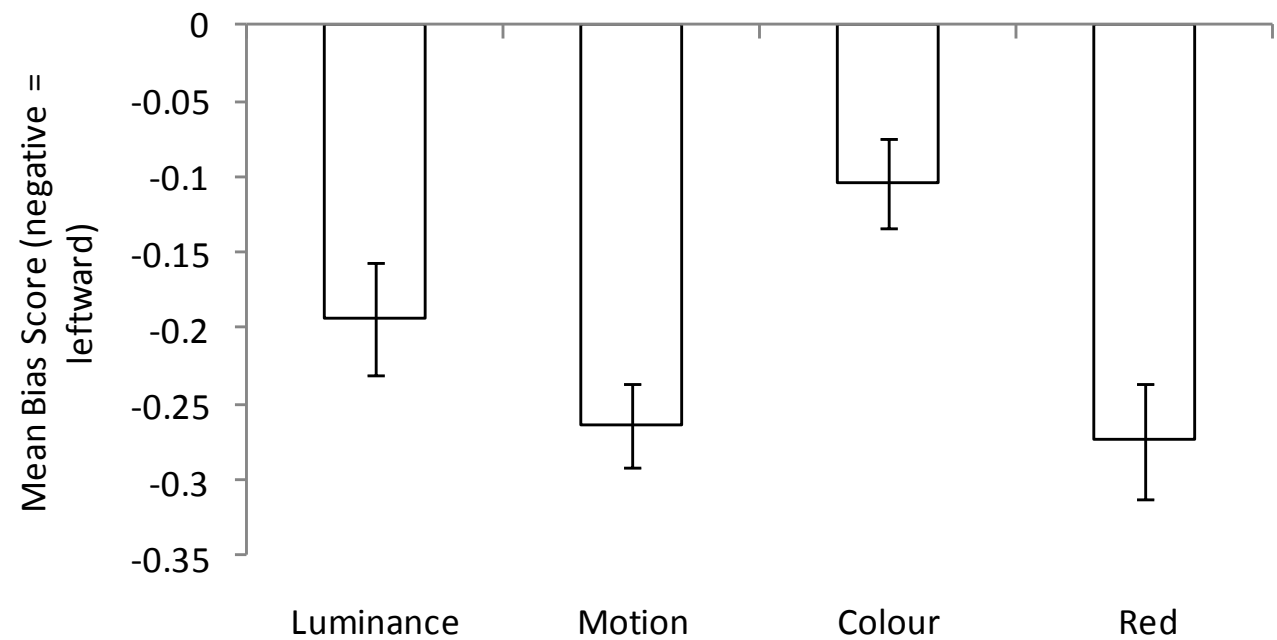

Figure 6-4. Mean bias scores for colour, motion, luminance and red tasks across all three experiments in Study 5. Error bars indicate the standard error of the mean.

Paired-samples $t$-tests were used to determine whether the strength of the left spatial bias differed between conditions. Significant differences emerged between colour and luminance, $t(128)=-3.169, p=.002$, and between colour and motion, $t(131)=3.741, p<.001$. Both luminance and motion exhibited stronger left biases than colour. No difference was seen between the luminance and motion conditions $(t(88)=.079, p=.937)$. 


\section{Discussion}

As hypothesized there was no significant bias seen on the isoluminant blue-yellow task. It can be seen that the colour red should not be used to examine perceptual asymmetries in spatial attention due to its ability to preferentially capture attention (Bellizzi \& Hite, 1992; Clynes, 1977; Elliot \& Maier, 2007; Elliot et al., 2007; Lu \& Zhou, 2005; Maier et al., 2009; Mehta \& Zhu, 2009; Tchernikov \& Fallah, 2010; Wilson, 1966). When the isoluminant colours blue and yellow were used, no bias emerged and when these data were combined with the green data from studies 1 and 2 and compared with luminance and motion scores, a significantly stronger left bias was seen on both motion and luminance compared to colour.

Further, the use of brief presentation times eliminated the leftward bias that was seen in studies 1 and 2 on the red judgments. When $150 \mathrm{msec}$ trials were used, the initial attentional bias that was seen for red judgments no longer occurred. This suggests that the left bias that was seen on prolonged trials results primarily from the longer viewing time as opposed to the processing of colour information. The left bias persisted on the motion task when it was completed under brief viewing conditions, illustrating that a different mechanism was underlying the leftward bias on the red judgments. If the same neural mechanisms were responsible for both biases, we would not expect to see a time-based change on only one measure (colour) and not the other (motion).

This provides support for the suggestion that the magnocellular pathway, leading to dorsal stream processing allows perceptual asymmetries to emerge, whereas ventral stream processing decreases the strength of the left spatial bias. No significant bias emerged on the greyscales measure in Study 3; however males in this experiment did not exhibit a significant bias, which accounted for the overall lack of bias on this task. As there were only eight males, this result cannot be generalized beyond the current study. 


\section{General Discussion}

It has been shown that the two visual streams possess different processing advantages, such that the ventral visual stream and parvocellular pathway are superior at processing colour and the magnocellular pathway and dorsal visual stream primarily process motion (Nieuwenhuis et al., 2008). Tasks incorporating either motion or colour, which were modifications of the greyscales task (Nicholls et al., 1999), were designed to target either dorsal or ventral stream processing. The motion task demonstrated a significant leftward bias. The bias on this task was comparable to that seen on the greyscales task, with the mean being slightly larger on the motion task, but not significantly so. Although the colour task also showed a significant leftward bias overall, this bias was significantly weaker than that seen on the motion or luminance tasks. This was consistent with the hypothesis that the bias on this task might not disappear completely as colour information has been shown to be accessible during dorsal stream tasks (i.e., White et al., 2006).

The lower VF, peripersonal space and dorsal stream processing are hypothesized to be associated and the ventral stream is thought to be advantaged at processing the upper VF and extrapersonal space (Previc, 1990). Although the relation of these three systems has not been demonstrated, Weiss et al. (2000) have shown primarily dorsal stream activation during peripersonal space processing and ventral stream during extrapersonal space processing. Thomas and Elias (2010) did not observe any space-based differences in perceptual asymmetries as a stronger left bias was seen in the lower VF across both distances; however it was suggested that a more direct manipulation of visual stream processing might better assess why VF differences occur in perceptual asymmetries. 
The dorsal stream and the lower VF are thought to be related (Previc, 1990), and as Thomas and Elias (2010) observed a stronger left bias in the lower VF, it follows that the dorsal stream is preferential in the emergence of the leftward bias. As the leftward bias persisted on the motion task, it suggests that the dorsal stream is preferential in the emergence of perceptual asymmetries. Dorsal stream processing might explain why the strength of the left bias is influenced by upper or lower VF presentation.

The findings from the first study indicate that the colours green, blue and yellow do not capture attention in the same manner in which red does. This finding is consistent with prior research that has suggested that the colour red captures attention more readily than other colours do (Bellizzi \& Hite, 1992; Clynes, 1977; Elliot \& Maier, 2007; Elliot et al., 2007; Lu \& Zhou, 2005; Maier et al., 2009; Mehta \& Zhu, 2009; Tchernikov \& Fallah, 2010; Wilson, 1966). As such, isoluminant blue and yellow are more appropriate when looking at attention-based differences in dorsal and ventral visual stream contribution. The use of brief presentation times clarified that red draws attention in a different manner during prolonged viewing as no bias was seen for red judgments during brief viewing.

As the magnocellular pathway promotes spatial segregation (Goodale \& Westwood, 2004; Livingstone \& Hubel, 1988; Nieuwenhuis et al., 2008) and the dorsal stream is primarily responsible for spatial judgments (Goodale \& Milner, 1992), it follows that the strength of the left bias on the greyscales task would be mediated by the magnocellular pathway. The current studied demonstrated that visual stream processing differences provide a possible explanation for the existence of upper and lower VF differences in perceptual asymmetries. Future research should make use of the motion and colour tasks developed here to examine VF differences in 
spatial biases. Presentation of these tasks to the upper and lower VF separately would provide additional evidence for dorsal stream involvement in the emergence of perceptual asymmetries. 


\section{CHAPTER 7 \\ DISCUSSION, CONCLUSIONS AND FUTURE DIRECTIONS}

\section{Discussion and Conclusions}

Neurologically normal individuals exhibit a leftward bias on various visuospatial tasks, including the greyscales task. Although this bias has been shown reliably, the neural mechanisms underlying this bias remain relatively unknown. Further, the context under which the bias occurs is unclear, as are precisely which factors influence the strength of the left bias. Several questions were addressed in order to better understand which factors influence perceptual asymmetries.

Prior work in the area had not addressed whether lateralized bumping could be observed on more "real-world" measures or whether it was only seen on laboratory measures (i.e., Nicholls et al. 2007; 2008). Although upper and lower VF differences had been observed on other tasks, I wanted to examine whether these could be seen on the greyscales task as well. I also wanted to know how length of presentation time influenced the strength of the left spatial bias in the upper and lower VFs. The issue of whether space-based differences would occur on the greyscales measure and how they might interact with VF differences was investigated. Previously, it had been shown that both object- and space-based biases contribute to the leftward bias, but it remained unknown what, specifically, the contributions of each one were. In addition, I questioned whether stimulus type (actual objects vs. greyscales) would influence which frame of reference contributes more to the left bias. Lastly, it was thought that it might be possible to decrease the left bias by targeting dorsal and ventral stream processing separately through the use of motion or colour.

A computer-based route following task was designed to investigate a more "real-world" perceptual asymmetry, referred to as lateralized bumping. Upper and lower VF differences were also examined using both this measure and the greyscales task. As a relation has been suggested 
between the lower VF and peripersonal space, and the upper VF and extrapersonal space (Previc, 1990), the greyscales task was presented in both spaces to see whether VF differences were related to space-based differences. In order to look at their unique contributions, object- and space-based biases were compared on the greyscales task (a spatial measure) and the object luminosity task (an object-based measure). The influence of the location of one image relative to the other was also examined to see whether biases would be stronger if images were partially overlapping than if there was no overlap. Following up on prior work, the length of presentation time was manipulated to see whether this would influence VF differences on the greyscales task. Finally, dorsal and ventral stream processing differences were investigated to see whether the left bias could be weakened or remain unchanged by incorporating isoluminant colour or motion into the task.

I demonstrated that the strength of the left bias differed depending on whether the computer-based route following task was presented centrally or whether it was presented to either the upper or the lower VF. This was the first indication that upper and lower VF differences previously observed on line bisection (Barrett et al., 2000; McCourt \& Garlinghouse, 2000; McCourt \& Jewell, 1999) could also be seen on a more real-world measure, increasing the ecological validity of these findings. This is significant as it shows the importance of better understanding these VF differences. Interestingly, perceptual asymmetries have been observed on real-world behaviours, such as navigation (Nicholls et al., 2007; 2008) and multiple choice responding (Nicholls et al., 2006a). It is possible that the previously observed leftward biases on multiple choice questions are even stronger in the lower VF, where most multiple choice questions are likely to be answered, further demonstrating the importance of better understanding upper and lower VF differences. 
In order to better understand VF differences, Thomas and Elias (2010) presented the greyscales task in the upper and lower VFs, and in peripersonal and extrapersonal space. We showed that the left bias was stronger in the lower VF in both peripersonal and extrapersonal space. This is a significant finding as there had been a lack of work studying upper and lower VF differences in perceptual asymmetries and the greyscales task had not previously been used to study them. VF differences were not associated with viewing distance, and although some work had shown a space-based dissociation (Bjoertomt et al., 2002; Garza et al., 2008; Longo \& Lourenco, 2006; Varnava et al., 2002), others failed to identify any differences (Longo \& Lourenco, 2006, Wilkinson \& Halligan, 2003). We concluded that although VF differences were not linked with space-based processing differences, the proposed relation between space-based and VF processing does not have strong empirical support either. Therefore, it is suggested that tasks targeting visual stream processing differences might better demonstrate why VF differences occur.

At this time, VF differences were examined in an alternate fashion by looking at objectand space-based biases. Spatial location influences the strength of the left bias, and although it is known that both object- and space-based biases contribute to the left bias (Nicholls et al. 2003; 2004a; 2006b; Orr and Nicholls 2005), how they interact with one another remains unclear. A left object-based bias was observed as images presented in the right VF showed a left bias. It is further suggested that an attentional bias to the central VF occurs as a leftward bias for images on the right side may represent the centremost image being chosen. These results are consistent with those of Orr and Nicholls (2005), who found a leftward object-based bias moderated by a stronger attentional bias to the centre VF. This would also explain the stronger left bias for 
images in the right VF, as opposed to those in the left VF, where a leftward bias would correspond with either a left spatial or a left object-based bias, but not with a central VF bias.

Symmetrical objects with central axes make it possible to objectively define the left side and the right side of an object separately from where it is located in space. Object-based biases emerged more strongly when actual objects were used in place of the greyscales, demonstrating that either object- or space-based biases will be stronger depending upon the type of stimuli that are used. However, results demonstrated a complex pattern wherein a leftward spatial bias was observed in one condition, a left object-based bias was observed in one condition and object- and space-based biases were both leftward in a third condition. Interestingly, the image selected most often was always located in the lower VF.

A similar bias toward the lower VF was detected in the left VF when the greyscales were used (Thomas \& Elias, in press). Both of these findings are consistent with prior reports of a stronger left bias in the lower VF (Barrett et al., 2000; Thomas \& Elias, 2010). It appears that object- and space-based coordinates interact with VF location, which leads either object- or space-based biases to be more prominent. As no bias was observed when the lower VF image was in the right VF and lit from the right, it suggests that the image in the lower VF must have at least one "left" characteristic for a bias to emerge. No bias was seen when leftward object- and space-based biases were congruent in the upper VF, which again suggests that the image must be placed in the lower VF for a bias to occur.

Prior research looking at upper and lower VF differences showed variable results, with some indicating a stronger left bias in the upper VF (McCourt \& Garlinghouse, 2000; McCourt \& Jewell, 1999) and others showing a stronger left bias in the lower VF (Barrett et al., 2000; Thomas \& Elias, 2010). The methodology employed in each study was not consistent, which 
could explain the mixed results. I used two presentation times to investigate whether or not this factor influenced the strength of the left bias differently in each VF. The left bias was stronger in the upper VF during brief presentation $(150 \mathrm{msec})$ and in the lower VF during prolonged presentation (10 sec maximum). This finding is significant as length of presentation time influenced the strength of the left bias, and this factor had not previously been considered in relation to VF differences.

It was also shown that participants' examined the left and lower VFs most often, consistent with a stronger left bias in the lower VF. There was a trend for a rightward bias in the upper VF during prolonged viewing, which is consistent with prior research showing an association between the lower and left VFs and the upper and right VFs. Further, eye movements toward upper VF targets are quicker than toward lower VF targets (e.g., Heywood \& Churcher, 1980; Honda \& Findlay, 1992). Upper VF targets could elicit quicker eye movements, making upper VF stimuli more detectable than lower VF ones. Individuals also elevate their eyes when attempting to scan information in memory (Kinsbourne, 1972), which could also explain a stronger left bias for upper VF presentations and a lack of bias in the lower VF during brief viewing.

Dorsal and ventral stream processing differences might underlie vertical attention differences (Drain \& Reuter-Lorenz, 1996; Post et al., 2006), in that tasks primarily carried out by the ventral pathway will show an accentuated upper VF bias and those that primarily activate the dorsal pathway will show a lower VF bias. In addition, the same neural mechanisms are thought to underlie perceptual asymmetries and hemispatial neglect, which can occur in the form of either upward or downward neglect. Patients with occipitoparietal lesions demonstrate lower VF neglect (Butter et al., 1989; Rapcsak et al., 1988), whereas occipitotemporal damage leads to 
upper VF neglect (Shelton et al., 1990). It was concluded that a more direct manipulation of dorsal and ventral processing differences was needed to provide further evidence for this suggestion.

In order to investigate potential dorsal and ventral visual stream contributions, modified greyscales tasks incorporating motion and colour were created. It was shown that both prolonged and brief motion tasks exhibited significant leftward biases, which were comparable to those typically seen on the greyscales task. It was also shown that a prolonged colour task exhibited a just significant left bias and a brief colour task did not show a significant bias. This was taken as evidence that the dorsal stream preferentially allows the left bias to emerge, whereas the ventral stream does not. Further, comparisons were made amongst the motion, colour and luminance (greyscales) tasks and it was shown that the biases on both the motion and luminance tasks were significantly stronger than the bias on the colour task. Interestingly, it was also shown that isoluminant red-green should not be used when studying visuospatial attention, as red captures attention more readily than other colours (i.e., Clynes, 1977; Elliot et al., 2007; Maier et al., 2009; Mehta \& Zhu, 2009; Tchernikov \& Fallah, 2010). It is recommended that isoluminant blue-yellow be used in place of red-green to avoid this confound.

The current results suggest that upper and lower VF differences in perceptual asymmetries are the result of primarily (although not exclusively) dorsal stream processing. The lower VF is preferentially processed by the dorsal stream and as the leftward bias is stronger in the lower VF, it follows that the dorsal stream facilitates the left bias. Various factors were shown to influence the strength of the left bias and the majority of the results can be explained in relation to visual stream processing differences. The most consistent finding across all experiments was that the leftward bias was stronger in the lower VF than in the upper VF. 
During prolonged presentation, the bias was stronger in the lower VF on the greyscales task when the task was performed in both peripersonal and extrapersonal space. On the object luminosity task, in all conditions that exhibited a significant bias, the image chosen was located in the lower VF. When the greyscale rectangles were shifted toward the left side to incorporate relative position, a significant bias was only seen when the shifted image was the lower one.

During brief presentation the left bias was stronger in the upper VF. On the computerbased route following task participants were instructed to complete the task as quickly and accurately as possible, and there were more collisions with the right side in the upper VF. During speeded presentation or when performing under temporal constraints, the leftward bias was stronger in the upper VF as opposed to the lower VF. This could be due to decreased accuracy in the upper VF under temporal constraints, which allows the bias to occur.

Hemispatial neglect patients tend to show the most severe neglect in the lower left VF (Rubens, 1985), which supports the occurrence of a stronger left bias toward this area of space among neurologically normal individuals. As illustrated by the data, the dorsal stream, as a continuation of the magnocellular pathway appears to be responsible for the left bias being stronger in the lower VF. The motion task exhibited a strong left bias and the colour task did not show the same bias, which further suggests that dorsal stream processing is preferable for the left bias to be seen.

\section{Future Directions}

\section{Follow-up Work}

Following the current research, there are questions that remain unanswered. I found that dorsal stream processing most likely underlies the left bias. I was able to demonstrate that a motion task allowed the left bias to emerge, whereas a colour task significantly weakened the 
bias. The stronger left bias seen in the lower VF on the greyscales task is thought to relate to preferential dorsal stream processing, as is the strong bias seen on a motion task. Future research should compare performance on the motion and colour tasks in the upper and lower VFs as this would strengthen the evidence in support of this relation. These comparisons should also incorporate presentation time differences as this factor also influenced VF differences. The results of these experiments could then shed light on how these differences impact real-world behaviours, such as facial communication, navigation and driving.

I showed that both space-based and object-based biases occur; but that these biases are not additive, nor do they interact in a straightforward way. I also showed that there is a bias toward stimuli in the lower VF, which likely also interacts with both object- and space-based coordinates. It is not clear how these biases all interact with one another and future research is needed to clarify under which circumstances either object- or space-based biases might preferentially contribute. Future research should also compare how the use of various types of stimuli, such as objects or greyscale images, lead either object- or space-based biases to be stronger.

I showed that VF differences are seen on the greyscales task, but also that these differences were not related to distance-based differences. Further I showed that length of presentation time affected the strength of the left bias differently in the upper and lower VFs. It remains unknown as to how these factors might interact with one another. Although distancebased differences were not seen when comparisons were made between the upper and lower VFs, it is possible that length of presentation time interacts with space in a similar way to how it interacts with VF presentation. 
The first study demonstrated that it was possible to elicit lateralized bumping on a computer-based route following task, and that both handedness and VF differences were seen on this task. What remains unknown is the reliability of these findings as this is the only study to date to employ this measure. This measure should be used again to further examine handedness differences and to better understand under which conditions VF differences emerge.

\section{New Directions for Future Work}

An interesting area that remains relatively unexplored is that of individual differences. Prior work has suggested that there may be sex differences in line bisection; however in most instances they have not been reported in perceptual asymmetries (see Jewell \& McCourt, 2000 for a review). Therefore, both male and female participants were included in most studies and no restrictions were placed upon who could participate and who could not. This allowed us to use a more random sampling procedure and in each instance data were examined for any potential performance differences between males and females. The only instance in which sex differences were observed was in the third experiment of Study 5, where male participants performed differently than females on the greyscales task. This finding was reported, but it was not felt that this factor impacted the overall results significantly. Further, as only eight males were included in this study, no generalizations beyond the current dataset were made. If an equal number of males and females were included in each study, reliable sex-based comparisons could be made and more conclusive evidence as to whether sex has an effect on perceptual asymmetries could be obtained.

Similarly, there has been some indication that handedness influences directional biases; however others have failed to observe any handedness differences (see Jewell \& McCourt, 2000 for a review). Once again, both left-handed and right-handed participants were included in most 
studies and data were examined for handedness differences. Participants were randomly sampled in all instances and left-handed participants were included in all studies in which they volunteered. In any instance where there were no left-handed participants, they were not deliberately excluded; I simply did not have any left-handed volunteers. Handedness differences were only identified in Study 1, which suggests it might be beneficial to compare handedness differences directly by including the same number of left-handed and right-handed participants. As the goal of the current research was to obtain data that were representative of the general population and not to examine handedness differences directly, it was not desirable to recruit these left-handed participants specifically, but instead to simply examine the data for any potential differences that could be used to guide future work in this area. In addition, it is possible that handedness interacts with sex as functional lateralization is influenced by both sex and handedness (Hiscock, Inch, Jacek, Hiscock-Kalil, \& Kalil, 1994; Hiscock, Israelian, Inch, Jacek, \& Hiscock-Kalil, 1995; Pujol, Deus, Losilla, \& Capdevila, 1999; Vogel, Bowers, \& Vogel, 2003). It would be of interest to examine an equal number of male and female, lefthanders and right-handers to compare these factors together in one study.

Although an overall leftward bias has been found on tasks examining perceptual asymmetries, it has also been shown that some participants consistently err toward the right side (Braun \& Kirk, 1999; Cowie \& Hamill, 1998; Dellatolas et al., 1996; Jewell \& McCourt, 2000; Manning et al., 1990; McCourt, 2001; McCourt, Freeman, Tahmahkera-Stevens, \& Chaussee, 2001; McCourt \& Olafson, 1997; Wilkinson \& Halligan, 2003). The term "right-shifter" has been used to refer to these individuals as they are biased toward the right side over a repeated number of trials, instead of the more typical left side (Manning et al., 1990). These individuals provide an interesting comparison to "left-shifters" as it is not clear what the true proportion of 
right-shifters is (McCourt, 2001; McCourt et al., 2001). The use of various neuroimaging techniques, such as functional magnetic resonance imaging or functional transcranial Doppler ultrasonography, would make it possible to compare activation patterns and lateralization differences among right- and left-shifters. Further, it would be useful to examine how rightshifters perform on measures of lateralized bumping as it is not known whether this population shows a different pattern of directional collisions. It may be the case that differing activation patterns can be reliably observed and that this underlies the direction of lateral biases. It has been proposed that complementarity of function underlies the left hemispheric and the right hemispheric activation differences seen for different types of tasks (Badzakova-Trajkov, Häberling, Roberts, \& Corballis, 2010; Bryden, 1990; Bryden, Hécaen, \& DeAgostini, 1983). This could explain why some participants tend to be biased toward the right side. Further, it could be of interest to study those individuals who do not show a reliable bias, as well as those individuals who do not show a consistent directional bias to see if any differences in activation patterns can explain why these individuals differ.

An interesting idea that would strengthen the results reported here would be to mask the greyscale images in place of using prolonged presentation. Masking paradigms have been employed by various other researchers to study visuospatial attention (i.e., Ansorge \& Heumann, 2006; Brisson \& Jolicoeur, 2007; Carlson, Fee \& Reinke, 2009; Pessoa, Kastner, \& Ungerleider, 2003). If the target (the greyscales images) was presented briefly, and then a similarly shaped mask was presented following a short delay (see Klotz \& Wolff, 1995 for example), it would strengthen the evidence in support of the task being purely attentional and not based on the participant's memory of the image. It is recommended that a mask of similar shape be used as reaction times are typically faster and the number of errors is smaller when the target and the 
mask are congruent (Klotz \& Wolff, 1995). This paradigm would eliminate concerns related to scanning and, more importantly, regarding mental representations that might have been formed. It is possible that during prolonged presentation a mental representation of the image is maintained and that participants are still able to compare the two images when they are no longer visible. Even with brief presentation it is possible that a mental representation is formed and the use of a masking paradigm would eliminate this possibility. Representational neglect (Bisiach \& Luzzatti, 1978) and representational pseudoneglect (McGeorge, Beschin, Colnaghi, Rusconi, \& Della Sala, 2007) have both been demonstrated, which further suggests that a masking paradigm would be very useful in studying perceptual asymmetries. This would provide an excellent means of examining how mental representations of images contribute to the biases typically observed in the more commonly employed paradigms.

Comparisons of upper and lower VF differences could have been improved through the use of specific instructions to maintain fixation in the centre of the VF, not only between trials, but also throughout the duration of each trial. In the $150 \mathrm{msec}$ presentation conditions this was not a concern as presentation times were too quick to allow for saccadic eye movements (NagelLeiby et al., 1990). A chin rest was used and participants were instructed to remain still, but during prolonged trials participants could have adjusted their eye position upward or downward to each stimulus location. It can be argued that upper and lower VF differences were no longer being examined during the prolonged trials as eye movements would negate each VF condition and that only in the $150 \mathrm{msec}$ presentation conditions were VF differences actually examined.

This is an admitted limitation of the current work as the eye-tracking data illustrated that participants were actively scanning the stimuli in each VF condition. Eye-tracking data also showed that participants were making eye movements only to the VF in which stimuli were 
located, suggesting that differences between the two were still being studied. It might be the case that one should not refer to the comparisons made in the current studies as VF differences per se. The results of the investigations reported herein indicate that differences do exist based on whether data are presented in the upper or lower portions of the screen, in an attempt to assess VF differences, but that these comparisons could be improved by having participants maintain fixation or by only employing $150 \mathrm{msec}$ presentation times. If controls such as these were in place, results could more definitively be referred to as upper and lower VF differences and would provide stronger evidence for the occurrence of these differences in perceptual asymmetries. Due to the lack of prior research in this area, it is believed that the current studies provide sufficient evidence to warrant further investigation, which improves upon the methods employed here.

In the final study a narrow angle luminance probe was used to match red and green, and blue and yellow colours for isoluminance. However, it is difficult to achieve isoluminance, even in the best laboratory conditions (Gur \& Akri, 1992). The exact ratio needed to produce isoluminance depends on the specific task that is being performed as well as the retinal locus of the image (Livingstone \& Hubel, 1987; Mullen, 1985). In addition, isoluminance varies between individuals (Livingstone \& Hubel, 1987; Mullen, 1985) and therefore it is difficult, when using one set of stimuli, to control for these differences among every individual observer. More precise methods of determining isoluminance that take into account these individual differences would increase the accuracy of the findings reported here. A method in which the isoluminance ratio for each participant was determined and then employed in the task would strengthen the evidence in support of a dorsal stream advantage in perceptual asymmetries. However, as methods such as these are not typical in this area, I feel that the reported results are reliable, despite this limitation. A superior method taking into account these individual differences would strengthen 
the evidence in support of the differential contributions of each visual stream. Although the current work is not without limitation, the results reliably suggest that upper and lower VF differences exist in perceptual asymmetries and that these differences, which are likely due to magnocellular projections to the dorsal stream, warrant further investigation. The importance of this work can be clearly seen when looking at the impact that spatial biases play in everyday life. Many things that we do with relative frequency, such as choosing a seat in the movie theatre, relying on facial communication when interacting with others, navigating and driving might all be influenced by the biases reported here. This demonstrates how the current work applies to our understanding of not only upper and lower VF differences on the greyscales task, but also how this work might relate to biases we exhibit every day. 


\section{CHAPTER 8}

\section{LIST OF REFERENCES}

Amenedo, E., Pazo-Alvarez, P., \& Cadaveira, F. (2007). Vertical asymmetries in pre-attentive detection of changes in motion direction. International Journal of Psychophysiology, 64, 184-189.

Ansorge, U., \& Heumann, M. (2006). Shifts of visuospatial attention to invisible (metacontrastmasked) singletons: Clues from reaction times and event-related potential. Advances in Cognitive Psychology, 2, 61-76.

Badzakova-Trajkov, G., Häberling, I.S., Roberts, R.P., \& Corballis, M.C. (2010). Cerebral asymmetries: Complementary and independent processes. Public Library of Science One, $5,1-9$

Barrett, A.M., Crosson, B., Crucian, G.P., \& Heilman, K.M. (2000). Horizontal line bisections in upper and lower body space. Journal of the International Neuropsychological Society, 6, 455-459.

Bellizzi, J.A., \& Hite, R.E. (1992). Environmental color, consumer feelings, and purchase likelihood. Psychology and Marketing, 9, 347-363.

Bisiach, E., \& Luzzatti, C. (1978). Unilateral neglect of representational space. Cortex, 14, 129133.

Bjoertomt, O., Cowey, A., \& Walsh, V. (2002). Spatial neglect in near and far space investigated by repetitive transcranial magnetic stimulation. Brain, 125, 2012-2022.

Bourassa, D.C., McManus, I.C., \& Bryden, M.P. (1996). Handedness and eye-dominance: A meta-analysis of their relationship. Laterality, 1, 5-34. 
Bowers, D., \& Heilman, K.M. (1980). Pseudoneglect: Effects of hemispace on a tactile line bisection task. Neuropsychologia, 18, 491-498.

Bradshaw, J.L., Nettleton, N.C., Nathan, G., \& Wilson, L. (1985). Bisecting rods and lines: Effects of horizontal and vertical posture on left-side underestimation by normal subjects. Neuropsychologia, 23, 421-425.

Brain, W.R. (1941). Visual disorientation with special reference to lesions of the right cerebral hemisphere. Brain, 64, 244-272.

Braun, J.G., \& Kirk, A. (1999). Line bisection performance of normal adults: Two subgroups with opposite biases. Neurology, 53, 527-532.

Brisson, B., \& Jolicoeur, P. (2007). Electrophysiological evidence of central interference in the control of visuospatial attention. Psychonomic Bulletin \& Review, 14, 126-132.

Brodie, E.E., \& Pettigrew, L.E.L. (1996). Is left always right? Directional deviations in visual line bisection as a function of hand and initial scanning direction. Neuropsychologia, 34, 467-470.

Bryden, M.P. (1990). Choosing sides: The left and the right of the normal brain. Canadian Psychology, 31, 297-309.

Bryden, M.P., Hécaen, H., \& DeAgostini, M. (1983). Patterns of cerebral organization. Brain and Language, 20, 249-262.

Butter, C.M., Evans, J., Kirsch, N., \& Kewman, D. (1989). Altitudinal neglect following traumatic brain injury: A case report. Cortex, 25, 135-146.

Cameron, E.L., Tai, J.C., \& Carrasco, M. (2002). Covert attention affects the psychometric function of contrast sensitivity. Vision Research, 42, 949-967. 
Caramazza, A., \& Hillis, A. (1990). Spatial representation of words in the brain implied by studies of a unilateral neglect patient. Nature, 346, 267-269.

Carlson, J.M., Fee, A.L., \& Reinke, K.S. (2009). Backward masked snakes and guns modulate spatial attention. Evolutionary Psychology, 7, 534-544.

Carrasco, M., Williams, P.E., \& Yeshurun, Y. (2002). Covert attention increases spatial resolution with or without masks: support for signal enhancement. Journal of Vision, 2, 467-479.

Chokron, S., Bartolomeo, P., Perenin, M., Helft, G., \& Imbert, M. (1998). Scanning direction and line bisection: A study of normal subjects and unilateral neglect patients with opposite reading habits. Cognitive Brain Research, 7, 173-178.

Christman, S. (1993). Local-global processing in the upper versus lower visual fields. Bulletin of the Psychonomic Society, 31, 275-278.

Christman, S.D., \& Niebauer, C.L. (1997). The relation between left-right and upper-lower visual field asymmetries (or: what goes up goes right, while what's left lays low). In S. D. Christman (Ed.), Cerebral Asymmetries in Sensory and Perceptual Processing (pp.263296). Amsterdam: Elsevier Science B. V.

Çiçek, M., Deouell, L.Y., \& Knight, R.T. (2009). Brain activity during landmark and line bisection tasks. Frontiers in Human Neuroscience, 3, 1-8.

Clynes, M. (1977). Sentics: The touch of emotions. Garden City, NY: Anchor Press.

Corbetta, M., Shulman, G.L., Miezin, F.M., \& Petersen, S.E. (1995). Superior parietal cortex activation during spatial attention shifts and visual feature conjunction. Science, 270, 802-805. 
Cowie, R., \& Hamill, G. (1998). Variation among nonclinical subjects on a line-bisection task. Perceptual and Motor Skills, 86, 834.

Dellatolas, G., Vanluchene, J., \& Coutin, T. (1996). Visual and motor components in simple line bisection: An investigation in normal adults. Cognitive Brain Research, 4, 49-56.

Diekamp, B., Regolin, L., Güntürkün, O., \& Vallortigara, G. (2005). A left-sided visuospatial bias in birds. Current Biology, 15, R372-R373.

Drain, M., \& Reuter-Lorenz, P.A. (1996). Vertical orienting bias: Evidence for attentional bias and 'neglect' in the intact brain. Journal of Experimental Psychology: General, 125, 139158.

Driver, J., Baylis, G.C., Goodrich, S.J., \& Rafal, R.D. (1994). Axis-based neglect of visual shapes. Neuropsychologia, 32, 1353-65.

Driver, J., Baylis, G.C., \& Rafal, R.D. (1992). Preserved figure-ground segregation and symmetry perception in visual neglect. Nature, 360, 73-74.

Driver, J., \& Halligan, P.W. (1991). Can visual neglect operate in object-centered co-ordinates? An affirmative single-case study. Cognitive Neuropsychology, 8, 475-496.

Dufour, A., Touzalin, P., \& Candas, V. (2007). Time-on-task effect in pseudoneglect. Experimental Brain Research, 176, 532-537.

Edgar, G., \& Smith, A. (1990). Hemifield differences in perceived spatial frequency. Perception, 19, 759-766.

Edwards, M., \& Badcock, D. (1993). Asymmetries in the sensitivity to motion in depth: A centripetal bias. Perception, 22, 1013-1023.

Efron, R., Yund, E., \& Nichols, D. (1987). Scanning the visual field without eye movements: A sex difference. Neuropsychologia, 25, 637-644. 
Efron, R., Yund, E., \& Nichols, D. (1990). Detectability as function of target location: Effects of spatial configuration. Brain and Cognition, 12, 102-116.

Elias, L.J., Bryden, M.P., \& Bulman-Fleming, M.B. (1998). Footedness is a better predictor than is handedness of emotional lateralization. Neuropsychologia, 36, 37-43.

Elliot, A.J., \& Maier, M.A. (2007). Color and psychological functioning. Current Directions in Psychological Science, 16, 250-254.

Elliot, A.J., Maier, M.A., Moller, A.C., Friedman, R., \& Meinhardt, J. (2007). Color and psychological functioning: The effect of red on performance attainment. Journal of Experimental Psychology: General, 136, 154-168.

Fink, G.R., Marshall, J.C., Shah, N.J., Weiss, P.H., Halligan, P.W., Grosse-Ruyken, M., ... Freund, H.J. (2000). Line bisection judgments implicate right parietal cortex and cerebellum as assessed by fMRI. Neurology, 54, 1324-1331.

Fink, G.R., Marshall, J.C., Weiss, P.H., \& Zilles, K. (2001). The neural basis of vertical and horizontal line bisection judgments: An fMRI study of normal volunteers. NeuroImage, 14, S59-S67.

Foley, J., \& McChesney, J. (1976). The selective utilization of information in the optic array. Psychological Research, 38, 251-265.

Foxe, J.J., McCourt, M.E., \& Javitt, D.C. (2003). Right hemisphere control of visuospatial attention: Line-bisection judgments evaluated with high-density electrical mapping and source analysis. NeuroImage, 19, 710-726.

Gainotti, G., Messerli, P., \& Tissot, R. (1972). Quantitative analysis of unilateral spatial neglect in relation to lateralisation of cerebral lesions. Journal of Neurology, Neurosurgery and Psychiatry, 35, 545-550. 
Garza, J.P., Eslinger, P.J., \& Barrett, A.M. (2008). Perceptual-attentional and motor intentional bias in near and far space. Brain and Cognition, 68, 9-14.

Giesbrecht, B., Kingstone, A., Handy, T.C., Hopfinger, J.B., \& Mangun, G.R. (2006) Functional neuroimaging of attention. In R. Cabeza, \& A. Kingstone (Eds.), Handbook of functional neuroimaging of cognition (pp. 85-112). Cambridge, MA: MIT Press.

Gitelman, D.R. (2002). ILAB: A program for postexperimental eye movement analysis. Behavior Research Methods, Instruments, \& Computers, 34, 605-612.

Goodale, M.A., \& Milner, A.D. (1992). Separate visual pathways for perception and action. Trends in Neurosciences, 15, 20-25.

Goodale, M.A., \& Westwood, D.A. (2004). An evolving view of duplex vision: Separate but interacting cortical pathways for perception and action. Current Opinion in Neurobiology, 14, 203-211.

Grimshaw, G.M., \& Keillor, J.M. (2000). Attentional and intentional factors in pseudoneglect. In M.K. Mandal, M.B. Bulman-Fleming, \& G. Tiwari (Eds.), Side bias: A neuropsychological perspective (pp. 331-346). Boston, MA: Kluwer Academic Publishers.

Grossi, D., Lepore, M., Napolitano, A., \& Trojano, L. (2001). On selective left neglect during walking in a child. Brain and Cognition, 47, 539-544.

Guez, J., Gargasson, J., Rigaudiere, F., \& O’Regan, J.K. (1993). Is there a systematic location for the pseudo-fovea in patients with central scotoma? Vision Research, 33, 1271-1279.

Gur, M., \& Akri, V. (1992). Isoluminant stimuli may not expose the full contribution of color to visual functioning: Spatial contrast sensitivity measurements indicate interaction between color and luminance processing. Vision Research, 32, 1253-1262. 
Halligan, P.W., Fink, G.R., Marshall, J.C., \& Vallar, G. (2003). Spatial cognition: Evidence from visual neglect. Trends in Cognitive Sciences, 7, 125-133.

Halligan, P.W., \& Marshall, J.C. (1991). Left neglect for near but not far space in man. Nature, $350,498-500$.

Halligan, P.W., \& Marshall, J.C. (1993). The history and clinical presentation of neglect. In I. H. Robertson, \& J. C. Marshall (Eds.), Unilateral neglect: Clinical and experimental studies (pp. 3-25). Hillsdale, NJ: Lawrence Erlbaum Associates Ltd.

Heilman, K. M. (1979). Neglect and related disorders. In K. M. Heilman \& E. Valenstein (Eds.), Clinical neuropsychology (pp. 268-307). New York, NY: Oxford University Press.

Heilman, K.M., Bowers, D., \& Watson, R.T. (1983). Performance on hemispatial pointing task by patients with neglect syndrome. Neurology, 33, 661-664.

Heilman, K., \& Valenstein, E. (1979). Mechanisms underlying hemispatial neglect. Annals of Neurology, 5, 166-170.

Heilman, K.M., Watson, R.T., \& Valenstein, E. (2003). Neglect and related disorders. In K.M. Heilman, \& E. Valenstein (Eds), Clinical Neuropsychology, (4 ${ }^{\text {th }}$ ed.) (pp. 296-346). New York, NY: Oxford University Press.

Heywood, S., \& Churcher, J. (1980). Structure of the visual array and saccadic latency: Implications for oculomotor control. Quarterly Journal of Experimental Psychology, 32, $335-341$.

Hillis, A.E., \& Caramazza, A. (1995). A framework for interpreting distinct patterns of hemispatial neglect. Neurocase, 1, 189-207.

Hillis, A.E., Rapp, B., Benzing, L., \& Caramazza, A. (1998). Dissociable coordinate frames of unilateral spatial neglect: "Viewer-centered" neglect. Brain and Cognition, 37, 491-526. 
Hiscock, M., Inch, R., Hawryluk, J., Lyon, P.J., \& Perachio, N. (1999). Is there a sex difference in human laterality? III. An exhaustive survey of tactile laterality studies from six neuropsychology journals. Journal of Clinical and Experimental Neuropsychology, 21, 17-28.

Hiscock, M., Israelian, M., Inch, R., Jacek, C., \& Hiscock-Kalil, C. (1995). Is there a sex difference in human laterality? II. An exhaustive survey of visual laterality studies from six neuropsychology journals. Journal of Clinical and Experimental Neuropsychology, $17,590-610$.

Holmes, G. (1918). Disturbances of visual orientation. British Journal of Ophthalmology, 2, 449506.

Holmes, G. (1919). Disturbances of visual space perception. British Medical Journal, 2, 230233.

Honda, H., \& Findlay, J. (1992). The increase in saccade latencies to the lower visual field. Perception \& Psychophysics, 52, 167-174.

Huitema, R.B., Brouwer, W.H., Hof, A.L., Dekker, R., Mulder, T., \& Postema, K. (2006). Walking trajectory in neglect patients. Gait \& Posture, 23, 200-205.

Hyman, Jr., I.E., \& Loftus, E.F. (1998). Errors in autobiographical memory. Clinical Psychology Review, 18, 933-947.

Jeerakathil, T.J., \& Kirk, A. (1994). A representational vertical bias. Neurology, 44, 703-706.

Jewell, G., \& McCourt, M.E. (2000). Pseudoneglect: A review and meta-analysis of performance factors in line bisection tasks. Neuropsychologia, 38, 93-110. 
Keller, I., Schindler, I., Kerkhoff, G., von Rosen, F., \& Golz, D. (2005). Visuospatial neglect in near and far space: Dissociation between line bisection and letter cancellation. Neuropsychologia, 43, 724-731.

Kinsbourne, M. (1970). The cerebral basis of lateral asymmetries in attention. Acta Psychologica, 3, 193-201.

Kinsbourne, M. (1972). Eye and head turning indicates cerebral lateralization. Science, 176, 539541.

Kleinman, J.T., Newhart, M., Davis, C., Heidler-Gary, J., Gottesman, R.F., \& Hillis, A.E. (2007). Right hemispatial neglect: Frequency and characterization following acute left hemisphere stroke. Brain and Cognition, 64, 50-59.

Klotz, W., \& Wolff, P. (1995). The effect of a masked stimulus on the response to the masking stimulus. Psychological Research, 58, 92-101.

Kosslyn, S.M. (1987). Seeing and imaging in the cerebral hemispheres: A computational approach. Psychological Review, 94, 148-175.

Krupp, D.B., Robinson, B.M., \& Elias, L.J. (2010). Free-viewing perceptual asymmetry for distance judgments: Objects in right hemispace are closer than they appear. International Journal of Neuroscience, 120, 580-582.

Lakha, L., \& Humphreys, G. (2005). Lower visual field advantage for motion segmentation during high competition for selection. Spatial Vision, 18, 447-460.

Livingstone, M.S., \& Hubel, D.H. (1987). Psychophysical evidence for separate channels for the perception of form, color, movement, and depth. The Journal of Neuroscience, 7, 34163468. 
Livingstone, M., \& Hubel, D. (1988). Segregation of form, color, movement, and depth:

Anatomy, physiology, and perception. Science, 240, 740-749.

Loftus, A., Vijayakumar, N., \& Nicholls, M. (2009). Prism adaptation overcomes pseudoneglect for the greyscales task. Cortex, 45, 537-543.

Longo, M.R. \& Lourenco, S.F. (2006). On the nature of near space: Effects of tool use and the transition to far space. Neuropsychologia, 44, 977-981.

Lu, S., \& Zhou, K. (2005). Stimulus-driven attentional capture by equiluminant color change. Psychonomic Bulletion \& Review, 12, 567-572.

Luh, K.E. (1995). Line bisection and perceptual asymmetries in normal individuals: What you see is not what you get. Neuropsychology, 9, 435-448.

Lundh, B., Lennerstrand, G., \& Derefeldt, G. (1983). Central and peripheral normal contrast sensitivity for static and dynamic sinusoidal gratings. Acta Ophthalmologica, 61, 171182.

Maier, M.A., Barchfeld, P., Elliot, A.J., \& Pekrun, R. (2009). Context specificity of implicit preferences: The case of human preference for red. Emotion, 9, 734-738.

Manning, L., Halligan, P.W., \& Marshall, J.C. (1990). Individual variation in line bisection: A study of normal subjects with application to the interpretation of visual neglect. Neuropsychologia, 28, 647-655.

Mattingley, J.B., Berberovic, N., Corben, L., Slavin, M.J., Nicholls, M.E.R., \& Bradshaw, J.L. (2004). The greyscales task: A perceptual measure of attentional bias following unilateral hemispheric damage. Neuropsychologia, 42, 387-394. 
Mattingley, J.B., Pierson, J.M., Bradshaw, J.L., Phillips, J.G., \& Bradshaw, J.A. (1993). To see or not to see: The effects of visible and invisible cues on line bisection judgements in unilateral neglect. Neuropsychologia, 31, 1201-1215.

McCourt, M.E. (2001). Performance consistency of normal observers in forced-choice tachistoscopic visual line bisection. Neuropsychologia, 39, 1065-1076.

McCourt, M., Freeman, P., Tahmahkera-Stevens, C., \& Chausee, M. (2001). The influence of unimanual response on pseudoneglect magnitude. Brain and Cognition, 45, 52-63.

McCourt, M.E., \& Garlinghouse, M. (2000). Asymmetries of visuospatial attention are modulated by viewing distance and visual field elevation: Pseudoneglect in peripersonal and extrapersonal space. Cortex, 36, 715-731.

McCourt, M.E., Garlinghouse, M., \& Slater, J. (2000). Centripetal versus centrifugal bias in visual line bisection: Focusing attention on two hypotheses. Frontiers in Bioscience, 5 , $58-71$.

McCourt, M.E., \& Jewell, G. (1999). Visuospatial attention in line bisection: Stimulus modulation of pseudoneglect. Neuropsychologia, 37, 843-855.

McCourt, M.E., \& Olafson, C. (1997). Cognitive and perceptual influences on visual line bisection: Psychophysical and chronometric analyses of pseudoneglect. Neuropsychologia, 35, 369-380.

McGeorge, P., Beschin, N., Colnaghi, A., Rusconi, M.L., \& Sala, S.D. (2007). A lateralized bias in mental imagery: Evidence for representational pseudoneglect. Neuroscience Letters, $421,259-263$.

Mehta, R., \& Zhu, R. (2009). Blue or red? Exploring the effect of color on cognitive task performances. Science, 323, 1226-1229. 
Mennemeier, M., Vezey, E., Chatterjee, A., Rapcszak, Z., \& Heilman, K.M. (1997).

Contributions of the left and right cerebral hemispheres to line bisection.

Neuropsychologia, 35, 703-715.

Milner, A.D., Brechmann, M., \& Pagliarini, L. (1992). To halve or to halve not: An analysis of line bisection judgements in normal subjects. Neuropsychologia, 30, 515-526.

Milner, A.D., Harvey, M., Roberts, R.C., \& Forster, S.V. (1993). Line bisection errors in visual neglect: Misguided action or size distortion? Neuropsychologia, 31, 39-49.

Mullen, K. (1985). The contrast sensitivity of human colour vision to red-green and blue-yellow chromatic gratings. The Journal of Physiology, 359, 381-400.

Murasugi, C., \& Howard, I. (1989). Human horizontal optokinetic nystagmus elicited by the upper versus lower visual fields. Visual Neuroscience, 2, 73-79.

Nagel-Leiby, S., Buchtel, H.A., \& Welch, K.M.A. (1990). Cerebral control of directed visual attention and orienting saccades. Brain, 113, 237-276.

Nichelli, P., Rinaldi, M., \& Cubelli, R. (1989). Selective spatial attention and length representation in normal subjects and in patients with unilateral spatial neglect. Brain and Cognition, 9, 57-70.

Nicholls, M.E.R., Bradshaw, J.L., \& Mattingley, J.B. (1999). Free-viewing perceptual asymmetries for the judgement of brightness, numerosity and size. Neuropsychologia, 37, 307-314.

Nicholls, M.E.R., Hughes, G., Mattingley, J.B., \& Bradshaw, J.L. (2004a). Are object- and space-based attentional biases both important to free-viewing perceptual asymmetries? Experimental Brain Research, 154, 513-520. 
Nicholls, M.E.R., Loftus, A., Mayer, K., \& Mattingley, J.B. (2007). Things that go bump in the right: The effect of unimanual activity on rightward collisions. Neuropsychologia, 45, 1122-1126.

Nicholls, M.E.R., Loftus, A., Orr, C., \& Barre, N. (2008). Rightward collisions and their association with pseudoneglect. Brain and Cognition, 68, 166-170.

Nicholls, M.E.R., Mattingley, J B., Berberovic, N., Smith, A., \& Bradshaw, J.L. (2004b). An investigation of the relationship between free-viewing perceptual asymmetries for vertical and horizontal stimuli. Cognitive Brain Research, 19, 289-301.

Nicholls, M.E.R., Mattingley, J.B., Bradshaw, J.L., \& Krins, P.W. (2003). Trunk- and headcentred spatial coordinates do not affect free-viewing perceptual asymmetries. Brain and Cognition, 53, 247-252.

Nicholls, M.E.R., Orr, C., Okubo, M., \& Loftus, A. (2006a). Satisfaction guaranteed: The effect of spatial biases on responses to likert scales. Psychological Science, 17, 1027-1028.

Nicholls, M.E.R., \& Roberts, G.R. (2002). Can free-viewing perceptual asymmetries be explained by scanning pre-motor or attentional biases? Cortex, 38, 113-136.

Nicholls, M.E.R., Smith, A., Mattingley, J.B., \& Bradshaw, J.L. (2006b). The effect of body and environment-centred coordinates on free-viewing perceptual asymmetries for vertical and horizontal stimuli. Cortex, 42, 336-346.

Niebauer, C., \& Christman, S. (1998). Upper and lower visual field differences in categorical and coordinate judgments. Psychonomic Bulletin and Review, 5, 147-151.

Nielsen, K.E., Intriligator, J., \& Barton, J.J.S. (1999). Spatial representation in the normal visual field. Neuropsychologia, 37, 267-277. 
Niemeier, M., \& Karnath, H. (2002). The exploration of space and objects in neglect. In H.

Karnath, D. Milner, \& G. Vallar (Eds.), The cognitive and neural bases of spatial neglect, (pp. 101-118). New York, NY: Oxford University Press.

Niemeier, M., Stojanoski, B., Singh, V.W.A., \& Chu, E. (2008). Paradoxical cross-over due to attention to high or low spatial frequencies. Brain and Cognition, 67, 115-125.

Nieuwenhuis, S., Jepma, M., La Fors, S., \& Olivers, C.N.L. (2008). The role of the magnocellular and parvocellular pathways in the attentional blink. Brain and Cognition, $68,42-48$.

Okubo, M. (2010). Right movies on the right seat: Laterality and seat choice. Applied Cognitive Psychology, 24, 90-99.

Okubo, M., \& Nicholls, M.E.R. (2006). A stimulus-dependent dissociation between the cerebral hemispheres under free-viewing conditions. Experimental Brain Research, 172, 49-56.

Orr, C.A., \& Nicholls, M.E.R. (2005). The nature and contribution of space- and object-based attentional biases to free-viewing perceptual asymmetries. Experimental Brain Research, $162,384-393$.

Payne, W. (1967). Visual reaction times on a circle about the fovea. Science, 155, 481-482.

Pennal, B. (1977). Human cerebral asymmetry in color discrimination. Neuropsychologia, 15, 563-568.

Pessoa, L., Kastner, S., \& Ungerleider, L.G. (2003). Neuroimaging studies of attention: From modulation of sensory processing to top-down control. The Journal of Neuroscience, 23, 3990-3998.

Pia, L., Neppi-Modona, M., \& Folegatti, A. (2010). Object-centred pseudoneglect for non-verbal visual stimuli. Experimental Brain Research, 200, 61-66. 
Posner, M.I., \& Petersen, S.E. (1990). The attention system of the human brain. Annual Review of Neuroscience, 13, 25-42.

Posner, M.I., \& Rothbart, M.K. (2007). Research on attention networks as a model for the integration of psychological science. Annual Review of Psychology, 58, 1-23.

Post, R.B., O’Malley, M.D., Yeh, T.L., \& Bethel, J. (2006). On the origin of vertical line bisection errors. Spatial Vision, 19, 505-527.

Prete, F.H. (1993). Stimulus configuration and location in the visual field affect appetitive responses by the praying mantis, Sphodromantis lineola (Burr.). Visual Neuroscience, 10, 997-1003.

Previc, F.H. (1990). Functional specialization in the lower and upper visual fields in humans: Its ecological origins and neurophysiological implications. The Behavioral and Brain Sciences, 13, 519-575.

Previc, F.H., \& Blume, J. (1993).Visual search asymmetries in three-dimensional space. Vision Research, 33, 2697-2704.

Previc, F.H., Breitmeyer, B.G., \& Weinstein, L. (1995). Discriminability of random-dot stereograms in three-dimensional space. International Journal of Neuroscience, 80, 247253.

Pujol, J., Deus, J., Losilla, J., \& Capdevila, A. (1999). Cerebral lateralization of language in normal left-handed people studied by functional MRI. Neurology, 52, 1038-1043.

Rapcsak, S.Z., Cimino, C.R., \& Heilman, K.M. (1988). Altitudinal neglect. Neurology, 38, 277281.

Raymond, J.E. (1994). Directional anisotropy of motion sensitivity across the visual field. Vision Research, 34, 1029-1037. 
Regan, D., Erkelens, C.J., \& Collewijn, H. (1986). Visual field defects for vergence eye movements and for stereomotion perception. Investigative Ophthalmology \& Visual Science, 27, 806-819.

Regolin, L. (2006). The case of the line-bisection: When both humans and chickens wander left. Cortex, 42, 101-103.

Reuter-Lorenz, P.A., Kinsbourne, M., \& Moscovitch, M. (1990). Hemispheric control of spatial attention. Brain and Cognition, 12, 240-266.

Rezec, A.A., \& Dobkins, K.R. (2004). Attentional weighting: A possible account of visual field asymmetries in visual search? Spatial Vision, 17, 269-293.

Rijsdijk, J., Kroon, J., \& van der Wildt, G. (1980). Contrast sensitivity as a function of position on the retina. Vision Research, 20, 235-241.

Robertson, I.H., Tegnér, R., Goodrich, S.J., \& Wilson, C. (1994). Walking trajectory and hand movements in unilateral left neglect: A vestibular hypothesis. Neuropsychologia, 32, 1495-1502.

Rolfe, M.H.S., Hamm, J.P., \& Waldie, K.E. (2008). Differences in paper-and-pencil versus computerized line bisection according to ADHD subtype and hand-use. Brain and Cognition, 66, 188-195.

Rubens, A. (1985). Caloric stimulation and unilateral visual neglect. Neurology, 35, 1019-1024.

Rubin, N., Nakayama, K., \& Shapley, R. (1996). Enhanced perception of illusory contours in the lower versus upper visual hemifields. Science, 271, 651-653.

Rueckert, L., \& McFadden, H.G. (2004). Context effects in pseudo-neglect measured with a free vision landmark task. Laterality, 9, 421-432. 
Rugani, R., Kelly, D.M., Szelest, I., Regolin, L., \& Vallortigara, G. (2010). Is it only humans that count from left to right? Biology Letters, 6, 290-292.

Sampaio, E., \& Chokron, S. (1992). Pseudoneglect and reversed pseudoneglect among lefthanders and right-handers. Neuropsychologia, 30, 797-805.

Scarisbrick, D.J., Tweedy, J.R., \& Kuslansky, G. (1987). Hand preference and performance effects on line bisection. Neuropsychologia, 25, 695-699.

Schiff, B.B., \& Truchon, C. (1993). Effect of unilateral contraction of hand muscles on perceiver biases in the perception of chimeric and neutral faces. Neuropsychologia, 31, 1351-1365.

Shelton, P.A., Bowers D., \& Heilman, K.M. (1990). Peripersonal and vertical neglect. Brain, $113,191-205$.

Siman-Tov, T., Mendelsohn, A., Schonberg, T., Avidan, G., Podlipsky, I., Pessoa, L., ... Hendler, T. (2007). Bihemispheric leftward bias in a visuospatial attention-related network. The Journal of Neuroscience, 27, 11271-11278.

Subbiah, I., \& Caramazza, A. (2000). Stimulus-centered neglect in reading and object recognition. Neurocase, 6, 13-31.

Tchernikov, I., \& Fallah, M. (2010). A color hierarchy for automatic target selection. Public Library of Science, 5, 1-4.

Thomas, N.A., \& Elias, L.J. (in press). Perceptual asymmetries in greyscales: Object-based versus space-based influences. Cortex.

Thomas, N.A., \& Elias, L.J. (2010). Do perceptual asymmetries differ in peripersonal and extrapersonal space? Journal of the International Neuropsychological Society, 16, 210214. 
Thomas, N.A., Stuckel, D., Gutwin, C., \& Elias, L.J. (2009). Directional collisions during a route-following task. Journal of the International Neuropsychological Society, 15, 225230.

Turnbull, O.H., \& McGeorge, P. (1998). Lateral bumping: A normal-subject analog to the behaviour or patients with hemispatial neglect? Brain and Cognition, 37, 31-33.

Vallar, G., \& Perani, D. (1986). The anatomy of unilateral neglect after right-hemisphere stroke lesions. A clinical/ct-scan correlation study in man. Neuropsychologia, 24, 609-622.

Varnava, A., McCarthy, M., \& Beaumont, J.G. (2002). Line bisection in normal adults: Direction of attentional bias for near and far space. Neuropsychologia, 40, 1372-1378.

Vingiano, W. (1991). Pseudoneglect on a cancellation task. The International Journal of Neuroscience, 58, 63-67.

Vogel, J.J., Bowers, C.A., \& Vogel, D.S. (2003). Cerebral lateralization of spatial abilities: A meta-analysis. Brain and Cognition, 52, 197-204.

Weiss, P.H., Marshall, J.C., Wunderlich, G., Tellmann, L., Halligan, P.W., Freund, H., ... Fink, G.R. (2000). Neural consequences of acting in near versus far space: A physiological basis for clinical dissociations. Brain, 123, 2531-2541.

White, B.J., Kerzel, D., \& Gegenfurtner, K.R. (2006). Visually guided movements to color targets. Experimental Brain Research, 175, 110-126.

Wilkinson, D., \& Halligan, P. (2003). The effects of stimulus size on bisection judgements in near and far space. Visual Cognition, 10, 319-340.

Wilson, G.D. (1966). Arousal properties of red versus green. Perceptual and Motor Skills, 23, 947-949. 
Winograd, E., Peluso, J.P., \& Glover, T.A. (1998). Individual differences in susceptibility to memory illusions. Applied Cognitive Psychology, 12, S5-S27. 


\section{APPENDIX A \\ WATERLOO HANDEDNESS AND FOOTEDNESS QUESTIONNAIRE-REVISED}

\section{PARTICIPANT QUESTIONNAIRE}

Age:

Sex (circle one): $\quad \mathbf{M} \quad \mathbf{F}$

If you are a student, what is your major? Year of study?

What was the first language you learned as a child? English French Chinese Other:

Do you have any hearing impairments? Yes No

Do you have any visual impairments (including colorblindness)? Yes No

If yes to either question, please specify:

What colour are your eyes? Blue Brown Green Hazel Violet Other:

What is your natural hair colour? Blond Brown Black Red Auburn Other:

Do you have any primary biological relatives (i.e., mother, father, brother, or sister) who are left-handed?

\section{Yes No Don't Know}

Do you have any biological extended family members (i.e., grandparents, biologically related aunts and uncles) who are left-handed? Yes No Don't Know

Please list any medications (including oral contraceptives) that you are currently taking:

Instructions: Please indicate your hand preference for the following activities by circling the appropriate response. If you always (i.e., $95 \%$ or more of the time) use one hand to perform the described activity, circle Ra or La (for right always or left always). If you usually (i.e., about $75 \%$ of the time) use one hand circle $\mathbf{R u}$ or $\mathbf{L u}$, as appropriate. If you use both hands equally often (i.e., you use each hand about $50 \%$ of the time), circle Eq.

$\begin{array}{llllll}\text { 1. With which hand would you use a pair of tweezers? } & \mathrm{La} & \mathrm{Lu} & \mathrm{Eq} & \mathrm{Ru} & \mathrm{Ra}\end{array}$

2. With which hand would you use a paintbrush to paint a wall? $\mathrm{La} \quad \mathrm{Lu} \quad \mathrm{Eq} \quad \mathrm{Ru} \quad \mathrm{Ra}$

$\begin{array}{llllll}\text { 3. Which hand would you use to pick up a book? } & \mathrm{La} & \mathrm{Lu} & \mathrm{Eq} & \mathrm{Ru} & \mathrm{Ra}\end{array}$

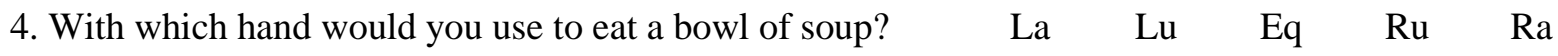

5. With which hand would you use the eraser on the end of a $\quad \begin{array}{lllllll}\mathrm{La} & \mathrm{Lu} & \mathrm{Eq} & \mathrm{Ru} & \mathrm{Ra}\end{array}$ pencil?

6. Which hand would you use to pick up a piece of paper? $\quad \begin{array}{llllll}\mathrm{La} & \mathrm{Lu} & \mathrm{Eq} & \mathrm{Ru} & \mathrm{Ra}\end{array}$

7. Which hand would you use to draw a picture?

$\mathrm{La} \quad \mathrm{Lu} \quad \mathrm{Eq} \quad \mathrm{Ru} \quad \mathrm{Ra}$

8. Which hand would you use to hammer a nail?

$\mathrm{La} \quad \mathrm{Lu} \quad \mathrm{Eq} \quad \mathrm{Ru} \quad \mathrm{Ra}$

9. Which hand would you use to insert a plug into an electrical $\quad \begin{array}{llllll}\mathrm{La} & \mathrm{Lu} & \mathrm{Eq} & \mathrm{Ru} & \mathrm{Ra}\end{array}$ outlet?

10. Which hand would you use to throw a ball?

11. In which hand would you hold a needle while sewing?

12. In which hand would you use to turn on a light switch?

$\begin{array}{lllll}\mathrm{La} & \mathrm{Lu} & \mathrm{Eq} & \mathrm{Ru} & \mathrm{Ra} \\ \mathrm{La} & \mathrm{Lu} & \mathrm{Eq} & \mathrm{Ru} & \mathrm{Ra} \\ \mathrm{La} & \mathrm{Lu} & \mathrm{Eq} & \mathrm{Ru} & \mathrm{Ra} \\ \mathrm{La} & \mathrm{Lu} & \mathrm{Eq} & \mathrm{Ru} & \mathrm{Ra}\end{array}$

13. Which hand do you use for writing? 


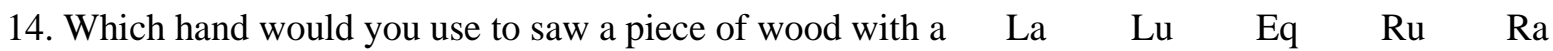
hand saw?

15. Which hand would you use to open a drawer? $\quad \begin{array}{llllll}\mathrm{La} & \mathrm{Lu} & \mathrm{Eq} & \mathrm{Ru} & \mathrm{Ra}\end{array}$ 16. Is there any reason (e.g., injury) why you have changed your hand preference for any of the above activities?

YES NO

17. Have you been given special training or encouragement to use a particular hand for certain activities?

YES NO

18. If you have answered YES to either Questions 16 or 17, please explain.

Instructions: Please indicate your foot preference for the following activities by circling the appropriate response. If you always (i.e., 95\% or more of the time) use one foot to perform the described activity, circle Ra or La (for right always or left always). If you usually (i.e., about $75 \%$ of the time) use one foot circle Ru or Lu (for right usually or left usually). If you use both feet equally often (i.e., you use each hand about $50 \%$ of the time), circle Eq. Please do not simply circle for all questions, but imagine yourself performing each activity in turn, and then mark the appropriate answer.

19. Which foot would you use to kick a stationary ball at a $\quad \begin{array}{llllll}\mathrm{La} & \mathrm{Lu} & \mathrm{Eq} & \mathrm{Ru} & \mathrm{Ra}\end{array}$ target straight ahead?

20. If you had to stand on one foot, which foot would it be? $\quad \begin{array}{lllllll}\mathrm{La} & \mathrm{Lu} & \mathrm{Eq} & \mathrm{Ru} & \mathrm{Ra}\end{array}$

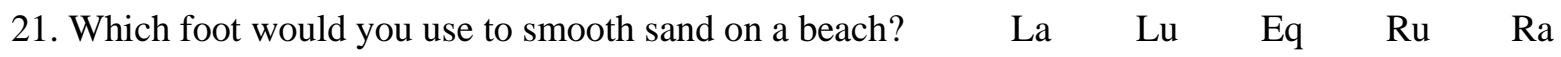

22. If you had to step up onto a chair, which foot would $\begin{array}{lllllll}\mathrm{La} & \mathrm{Lu} & \mathrm{Eq} & \mathrm{Ru} & \mathrm{Ra}\end{array}$ you place on the chair first?

23. Which foot would you use to stomp on a fast moving bug? $\quad \mathrm{La} \quad \mathrm{Lu} \quad \mathrm{Eq} \quad \mathrm{Ru} \quad \mathrm{Ra}$

24. If you were to balance on one foot on a railway track, which $\mathrm{La} \quad \mathrm{Lu} \quad \mathrm{Eq} \quad \mathrm{Ru} \quad \mathrm{Ra}$ foot would you use?

25. If you wanted to pick up a marble with your toes, which $\quad \begin{array}{lllllll}\mathrm{La} & \mathrm{Lu} & \mathrm{Eq} & \mathrm{Ru} & \mathrm{Ra}\end{array}$ foot would you use?

26. If you had to hop on one foot, which foot would you use? $\quad \begin{array}{lllllll}\mathrm{La} & \mathrm{Lu} & \mathrm{Eq} & \mathrm{Ru} & \mathrm{Ra}\end{array}$

27. Which foot would you use to help push a shovel into $\quad \begin{array}{lllllll}\mathrm{La} & \mathrm{Lu} & \mathrm{Eq} & \mathrm{Ru} & \mathrm{Ra}\end{array}$ the ground?

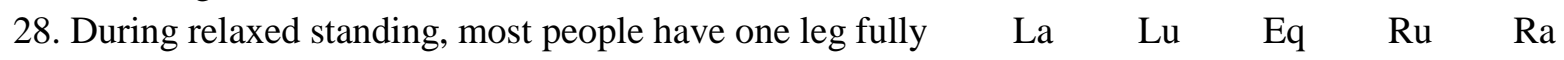
extended for support and the other slightly bent. Which leg do you have fully extended first?

29. Is there any reason (i.e. injury) why you have changed your foot preference for any of the above activities? $\quad$ YES NO 30. Have you ever been given special training or encouragement to use a particular foot for certain $\begin{array}{ll}\text { activities? } & \text { YES NO }\end{array}$ 31. If you have answered YES for either question 29 or 30, please explain:

This questionnaire was taken from Elias, Bryden, and Bulman-Fleming (1998). See reference chapter. 


\section{APPENDIX B}

STARTING VIEW OF THE COMPUTER-BASED ROUTE FOLLOWING TASK

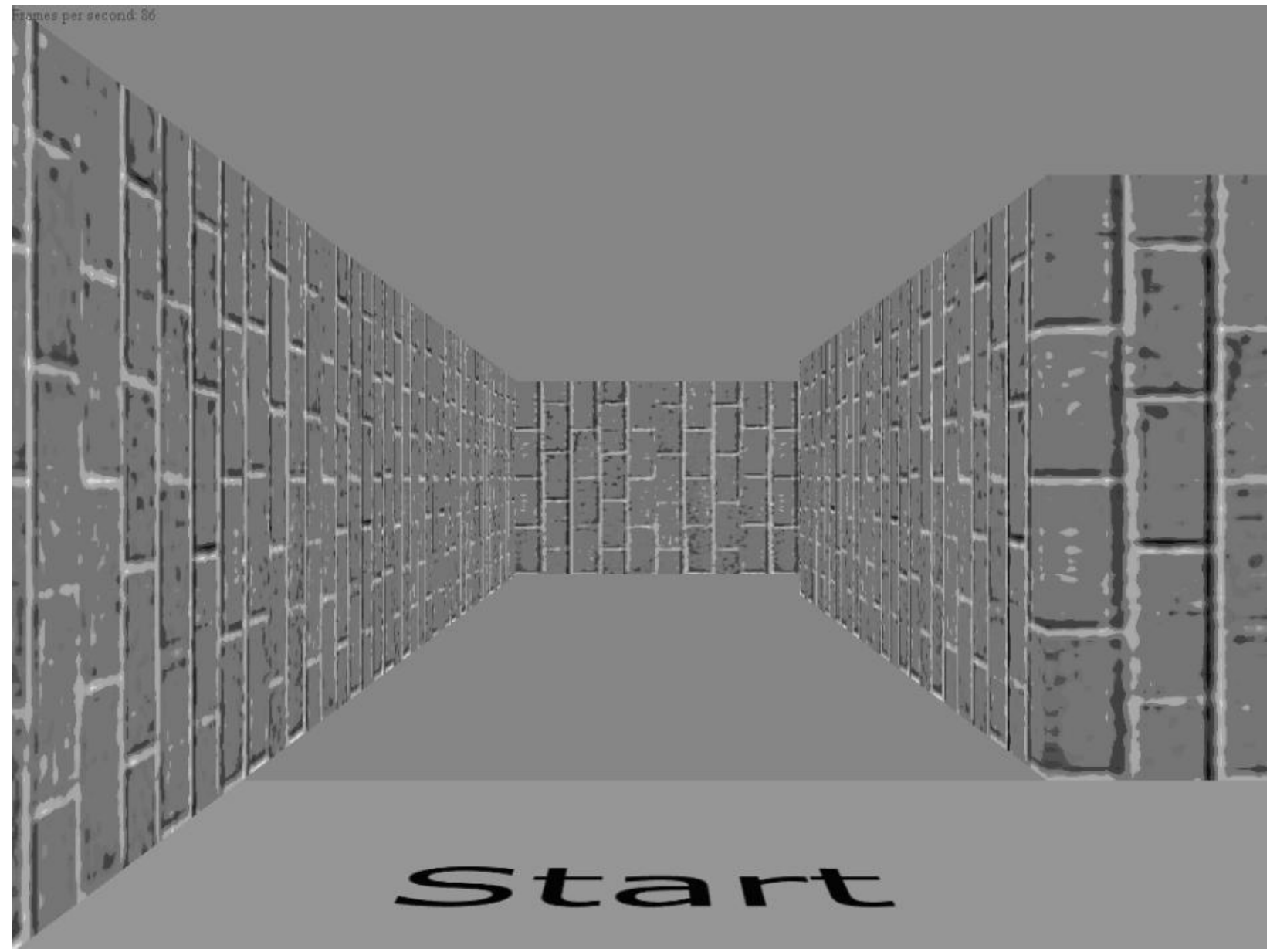




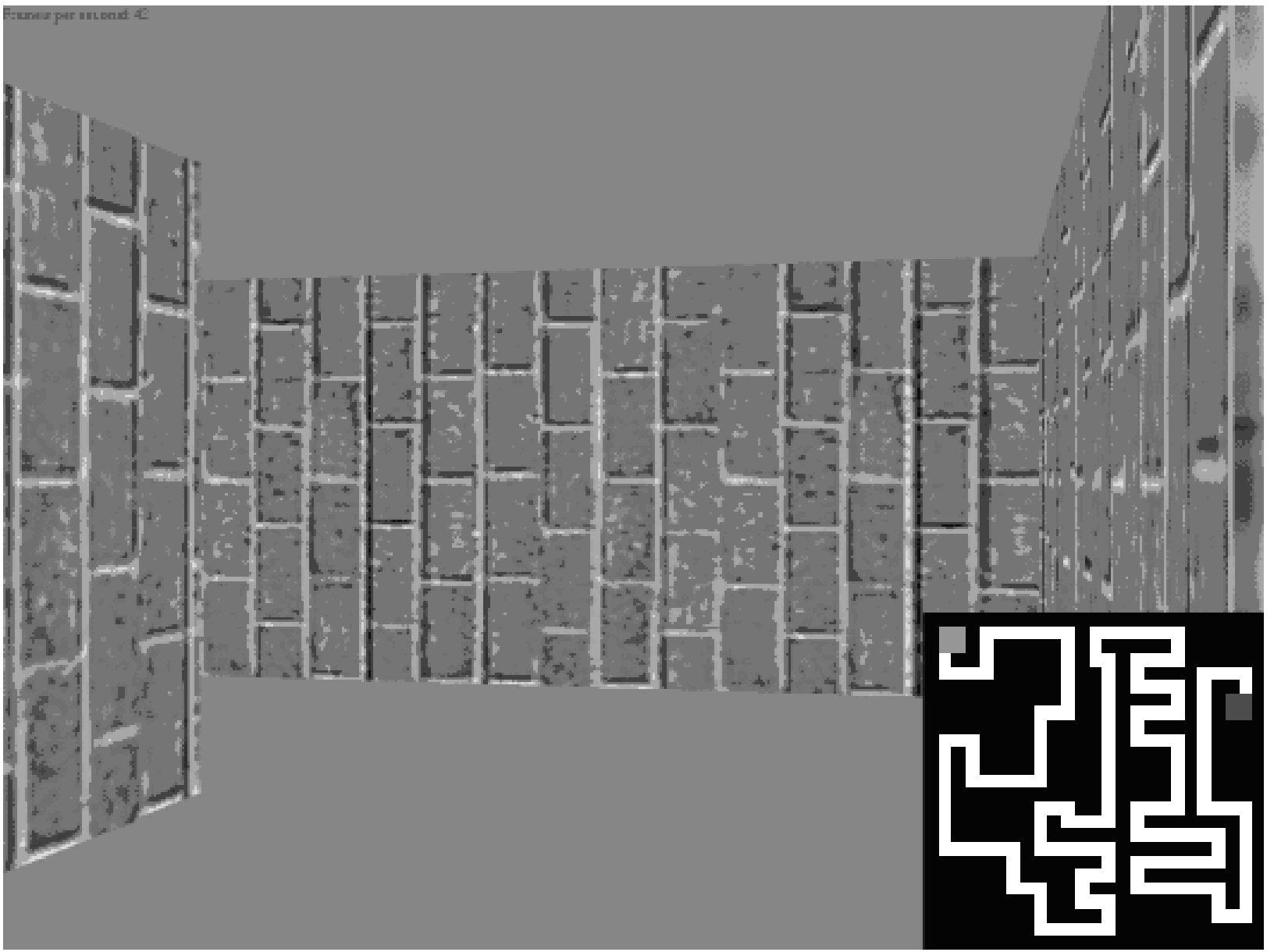

Inset is a sample bitmap of a pathway of the maze, with the shaded squares indicating the start and end points of the pathway. This inset was not visible to the participant; it is included currently to illustrate a sample pathway to the reader. 


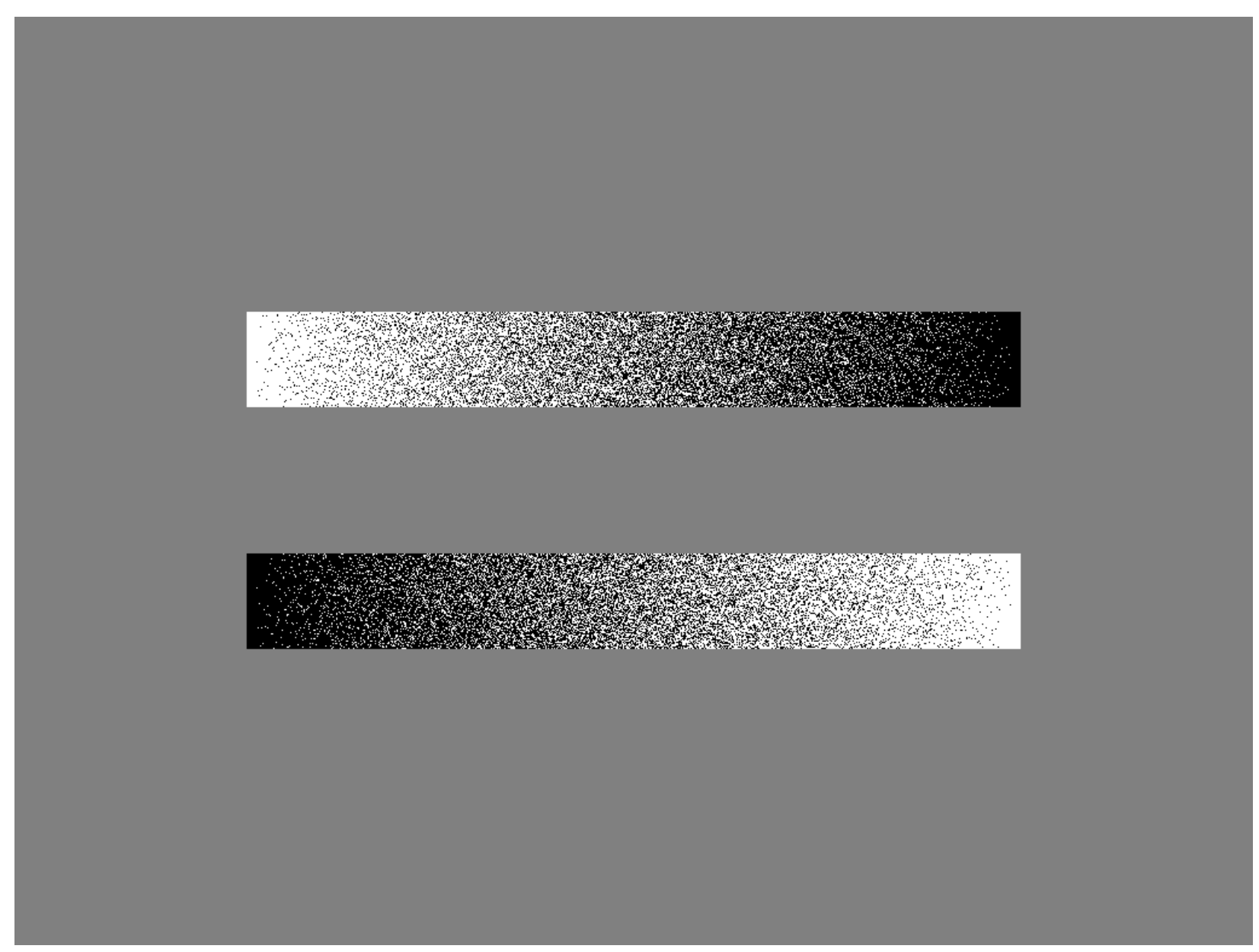

This task was created by Nicholls, Bradshaw, and Mattingley (1999). This sample illustrates how this task appears when it is presented in the centre of the visual field. 


\section{APPENDIX E \\ BUMPING QUESTIONNAIRE FROM DIRECTIONAL COLLISIONS DURING A ROUTE FOLLOWING TASK}

\section{Participant Questionnaire}

1. Have you ever accidentally bumped yourself against an object while concentrating on something else? YES NO

2. Please try to recall a specific incident when answering the following questions. If you cannot recall a specific incident, indicate this on the questionnaire and return it to the researcher.

a). Which PART of your body did you bump? (be specific)

b). Which SIDE of your body did you bump? (be specific)

c). Please describe the circumstances under which this incident occurred. Be as specific as possible. 


\section{APPENDIX F \\ CONSENT FORM FROM DIRECTIONAL COLLISIONS DURING A ROUTE FOLLOWING TASK}

You are invited to participate in a study entitled Can you Judge the Distance?(E37). Please read this form carefully, and feel free to ask questions you might have.

Researcher: Nicole Thomas, Department of Psychology, University of Saskatchewan

Dr. Lorin Elias, Department of Psychology, University of Saskatchewan

Purpose and Procedure: The purpose of this study is to investigate judgments of distance in real-world situations. Individuals will complete 4 computer tasks in which they must make judgments of distance as well as a demographic questionnaire. This study will take approximately 30 minutes.

Potential Risks: There are no known risks associated with this procedure.

Potential Benefits: This study should lead to further understanding of judgments of distance in a task that relates to real-world behaviour. As well, this study should increase understanding of why particular judgments of distance are made. Participants will gain experience with experimental psychology, and if they wish, they will be given the opportunity to learn the group results of the study.

Storage of Data: The data and consent forms will be stored securely at the University of Saskatchewan by Dr. L. Elias. Consent forms will be stored separately from the data materials so that it will not be possible to associate names with any given data. The data will be stored for a minimum of five years after completion of the study.

Confidentiality: Your data will be kept completely confidential and no personally identifying information will be linked to your data. All data will be reported in aggregated form.

Right to Withdraw: Your participation is voluntary, and you may withdraw from the study for any reason, at any time, without penalty of any sort and without the loss of your research credit. If you withdraw from the study at any time, any data that you have contributed will be destroyed.

Questions: If you have any questions concerning the study, please feel free to ask at any point; you are also free to contact the researchers at the numbers provided above if you have questions at a later time. This study has been approved on ethical grounds by the University of Saskatchewan Behavioural Research Ethics Board on January 29, 2007. Any questions regarding your rights as a participant may be addressed to that committee through the Ethics Office (966-2084). Out of town participants may call collect. You may contact the researcher if you wish to obtain the results of the study.

Consent to Participate: I have read and understood the description provided above; I have been provided with an opportunity to ask questions and my questions have been answered satisfactorily. I consent to participate in the study described above, understanding that I may withdraw this consent at any time. A copy of this consent form has been given to me for my records.

(Name of Participant)

(Signature of Participant)
(Date)

(Signature of Researcher) 


\title{
APPENDIX G \\ DEBRIEFING FORM FROM DIRECTIONAL COLLISIONS DURING A ROUTE FOLLOWING TASK
}

\author{
Study Title: Can you Judge the Distance?(E37)
}

Thank-you for participating in the study!

\begin{abstract}
It has been well established that normal individuals tend to underestimate the right side when performing distance judgment tasks or line bisection tasks. It is also true that participants prefer images that are lit from the left, or that are brighter or darker on the leftside. The final computer task that you completed looked at this by having you indicate whether you thought the image on the top or the bottom looked darker.
\end{abstract}

In an initial investigation of lateral bumping (collisions with objects), it was shown that it was more common to collide with objects on the right side of the body than the left side of the body. This was based on self-reported incidents by participants. A recent experiment showed that bumping appears to occur more on the right side in a more real-world based task as well. In this experiment participants walked through a doorway and any collisions were recorded. The current study is looking at lateral bumping by using a computer-based maze task. The number of collisions that were made on both the right and left sides while navigating the maze were recorded.

Initial investigations in this area have led to the hypothesis that a leftward bias is due to the activation of the right hemisphere during spatial tasks. However, there are many other explanations including scanning order (left to right), lateral biases in attention, or other perceptual effects. This study should help to clarify this further. 


\section{APPENDIX H}

SAMPLE STIMULI FROM THE GREYSCALES TASK WHEN PRESENTED TO EITHER THE UPPER OR THE LOWER VISUAL FIELD
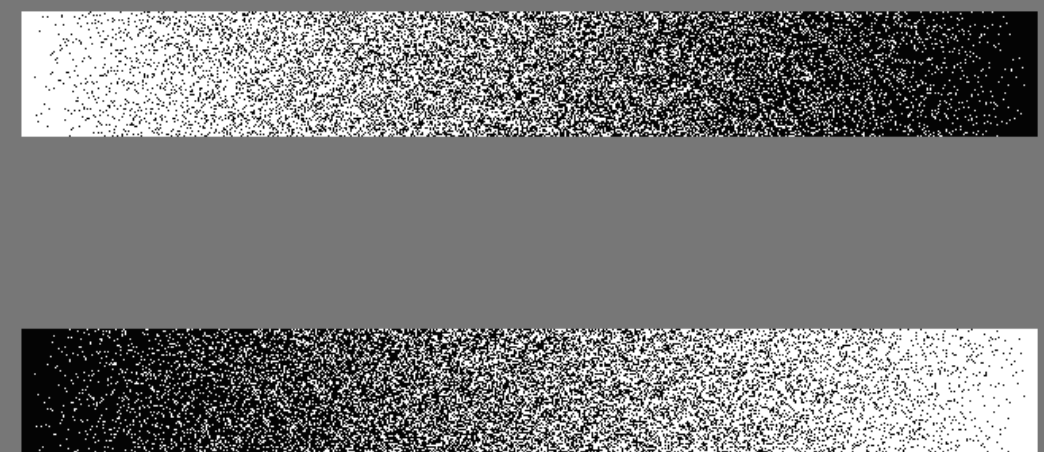


\section{APPENDIX I \\ CONSENT FORM FROM DO PERCEPTUAL ASYMMETRIES DIFFER IN PERIPERSONAL AND EXTRAPERSONAL SPACE?}

You are invited to participate in a study entitled Brightness Differences in Near and Far Space (X17). Please read this form carefully, and feel free to ask questions you might have.

Researchers: Nicole Thomas, Department of Psychology, University of Saskatchewan Dr. Lorin Elias, Department of Psychology, University of Saskatchewan

Purpose and Procedure: The purpose of this study is to investigate how judgments of luminance differ depending on whether they are presented in near or far space. Participants will complete 3 computer tasks where they must make judgments of luminance and distance as well as a demographic questionnaire, which will be used to describe the general characteristics of the entire group of participants. This study will take approximately 30 minutes.

Potential Risks: There are no known risks associated with this procedure; however you may receive no personal benefit from participation in the study. At the end of the study you will be given a sheet that better explains the nature of the study and you will be given a chance to ask any questions you might have.

Potential Benefits: This study should lead to further understanding of differences in judgments of luminance and distance depending on where in space the objects are located. As well, this study should help to understand how these particular judgments relate to the way the brain functions. Participants will gain experience with experimental psychology, and if they wish, they will be given the opportunity to learn the group results of the study.

Storage of Data: The data and consent forms will be stored securely at the University of Saskatchewan by Dr. L. Elias. Consent forms will be stored separately from the data materials so that it will not be possible to associate names with any given data. The data will be stored for five years after completion of the study and then destroyed beyond recovery.

Confidentiality: Your data will be kept completely confidential and no personally identifying information will be linked to your data. All data will be reported in aggregated form.

Right to Withdraw: Your participation is voluntary, and you may withdraw from the study for any reason, at any time, without penalty of any sort and without the loss of your research credit. If you withdraw from the study at any time, any data that you have contributed will be destroyed.

Questions: If you have any questions concerning the study, please feel free to ask at any point; you are also free to contact the researchers at the numbers provided above if you have questions at a later time. This study has been approved on ethical grounds by the University of Saskatchewan Behavioural Research Ethics Board on (Oct. 26, 2007). Any questions regarding your rights as a participant may be addressed to that committee through the Ethics Office (966-2084). Out of town participants may call collect. You may contact the researcher if you wish to obtain the results of the study.

Consent to Participate: I have read and understood the description provided above; I have been provided with an opportunity to ask questions and my questions have been answered satisfactorily. I consent to participate in the study described above, understanding that I may withdraw this consent at any time. A copy of this consent form has been given to me for my records.

(Name of Participant)

(Signature of Participant)
(Date)

(Signature of Researcher) 


\title{
APPENDIX J \\ DEBRIEFING FORM FROM DO PERCEPTUAL ASYMMETRIES DIFFER IN PERIPERSONAL AND EXTRAPERSONAL SPACE?
}

\author{
Study Title: Brightness Differences in Near and Far Space (X17)
}

Thank-you for participating in the study!

\begin{abstract}
It has been well established that the majority of people tend to underestimate the right side when making luminance judgments or bisecting lines. It is also true that participants tend to select images that are lit from the left as being either brighter or darker than identical but mirrorreversed images. You completed a computer task where you were asked to select which image appeared darker. The images that you viewed were of equal luminance; however most people select the image with the dark portion located on the left side as being darker overall.
\end{abstract}

A few studies have examined differences in line bisection related to where the line is located in space. It has been found that participants will bisect the same line in a different location depending on whether the line is located above or below eye-level. Some investigators have found that bisections are more leftward above eye-level, but others have found that they are more leftward below eye-level. Similar results are found when individuals are asked whether a line that is already bisected is bisected to the left or the right of the middle of the line. Despite these mixed findings, it is clear that differences exist depending on the location of the stimulus.

Some researchers suggest that these differences in perception above and below eye-level are related to information processing differences between far and near space, respectively. This is why you were asked to perform the task in far space (projected onto a screen) as well as near space (presented on a computer screen vertically in front of you and horizontally on the table top). The current study should increase understanding of these differences and may also increase understanding of how the human visual system perceives information. 


\section{APPENDIX K \\ SUMMARY OF PRESENTATION CONDITIONS PREVIOUSLY EMPLOYED IN PERCEPTUAL ASYMMETRIES STUDIES COMPARING OBJECT- AND SPACE-BASED INFLUENCES}
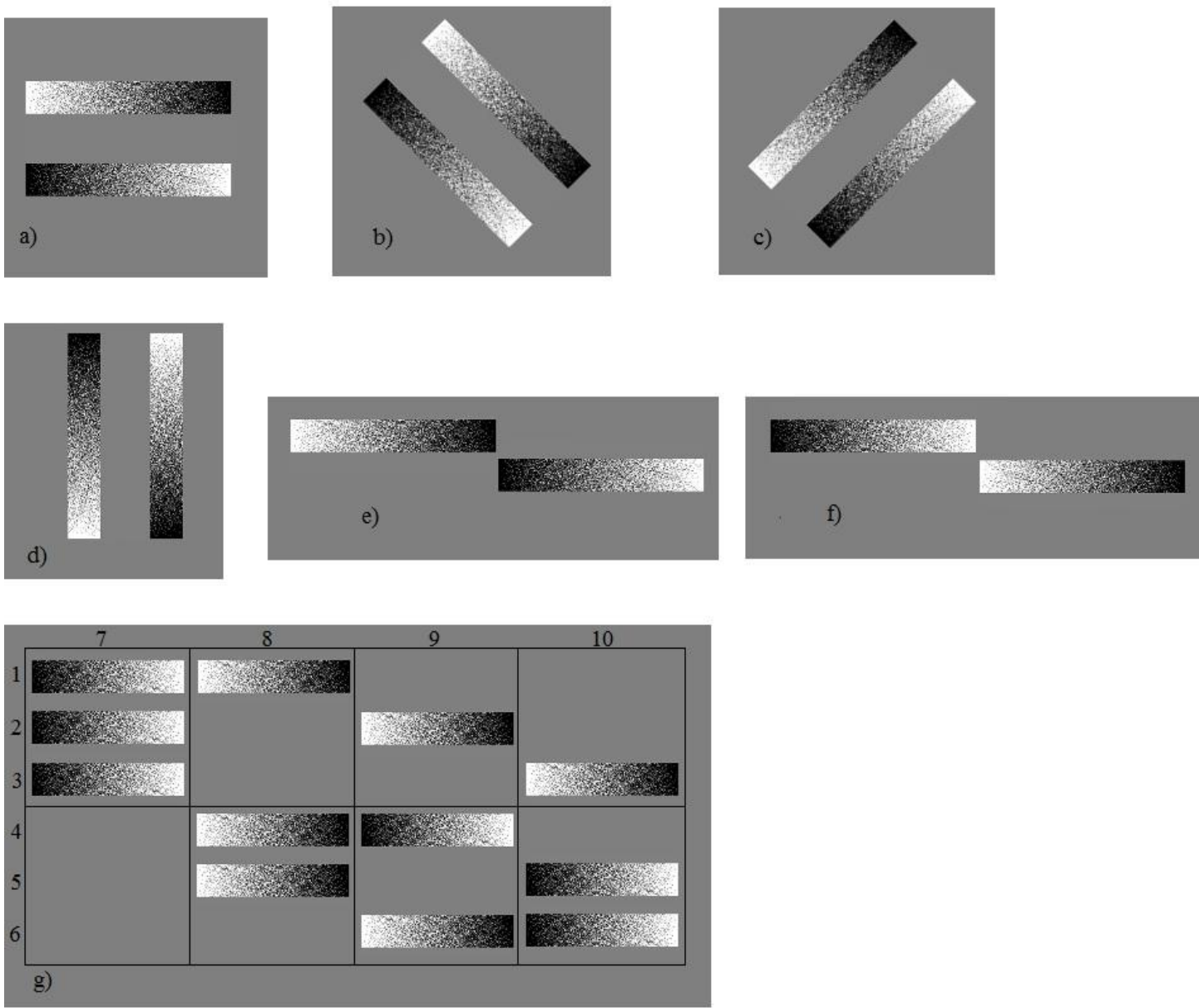

Description of object- versus space-based coordinates in each of the greyscales experiments. In a), the typical presentation of the greyscales, it can be seen that the left side of the object- is congruent with the left side of space, rendering it impossible to dissociate the two. In b) and c), the greyscales are rotated by $45^{\circ}$ positive or negative of $180^{\circ}$. This configuration is used to examine the interaction of vertical and horizontal biases. In d) the greyscales are presented vertically to examine upper and lower biases. In e) and f), object-based coordinates are either incongruent (as in e, where the dark portion is on the right) or congruent (as in $\mathrm{f}$, where the dark portion is on the left) with spatial coordinates. In $\mathrm{g}$ ), the conditions employed by Orr and Nicholls (2005) are depicted. The rows 1-6 show all possible location pairings of the two greyscale rectangles (i.e., 7 and 8, 7 and 9, 8 and 9, etc...). Rows 1-3 provide an example of congruent object- and space-based coordinates and rows 4-6 represent incongruent conditions (all pairings were actually presented in both congruent and incongruent conditions). 
APPENDIX L

SUMMARY OF PRESENTATION CONDITIONS USED IN PERCEPTUAL ASYMMETRIES IN GREYSCALES: OBJECT-BASED VERSUS SPACE-BASED INFLUENCES (STUDY 1)

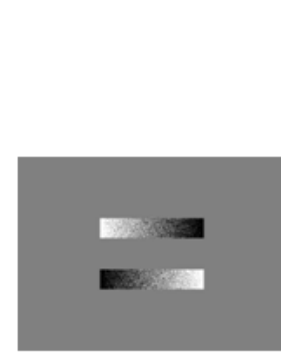

a)

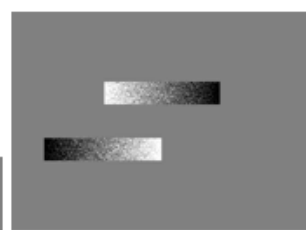

b)

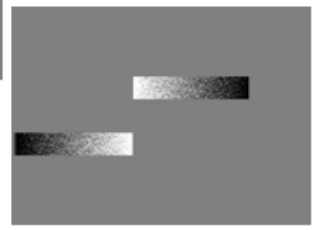

c)

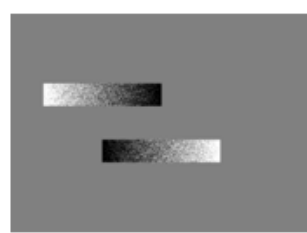

d)

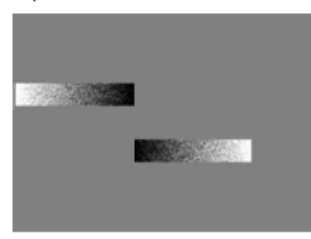

e)

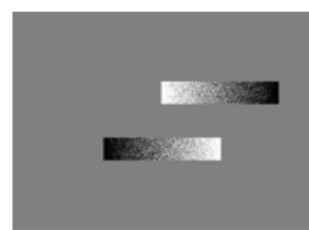

f)

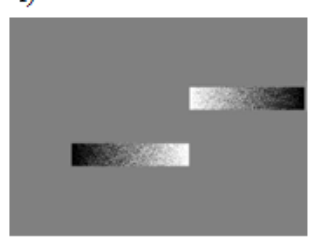

g)

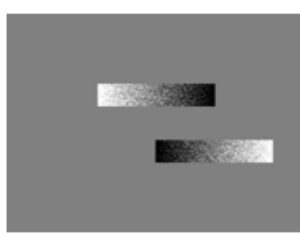

h)

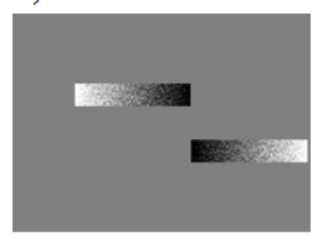

i) 


\section{APPENDIX M \\ CONSENT FORM FROM PERCEPTUAL ASYMMETRIES IN GREYSCALES: OBJECT- BASED VERSUS SPACE-BASED INFLUENCES (STUDY 1) AND UPPER AND LOWER VISUAL FIELD DIFFERENCES IN PERCEPTUAL ASYMMETRIES}

You are invited to participate in a study entitled Upper and Lower Visual Field Differences in Pseudoneglect (F34). Please read this form carefully, and feel free to ask questions you might have.

Researchers: Nicole Thomas, Department of Psychology, University of Saskatchewan

Dr. Lorin Elias, Department of Psychology, University of Saskatchewan

Purpose and Procedure: The purpose of this study is to investigate how judgments of luminance and distance differ depending on whether they are presented above or below eye level. Individuals will complete a computer task in which they must make judgments of luminance and distance as well as a demographic questionnaire. This study will take approximately 30 minutes. A special camera will also be used to track your eye movements for each trial

Potential Risks: There are no known risks associated with this procedure; however you may receive no personal benefit from participation in the study. At the end of the study you will be given a sheet that better explains the nature of the study and you will be given a chance to ask any questions you might have.

Potential Benefits: This study should lead to further understanding of differences in judgments of luminance and distance depending on where in space the objects are located. As well, this study should help to understand how these particular judgments relate to the way the brain functions. Participants will gain experience with experimental psychology, and if they wish, they will be given the opportunity to learn the group results of the study.

Storage of Data: The data and consent forms will be stored securely at the University of Saskatchewan by Dr. L. Elias. Consent forms will be stored separately from the data materials so that it will not be possible to associate names with any given data. The data will be stored for a minimum of five years after completion of the study. Confidentiality: Your data will be kept completely confidential and no personally identifying information will be linked to your data. All data will be reported in aggregated form.

Right to Withdraw: Your participation is voluntary, and you may withdraw from the study for any reason, at any time, without penalty of any sort and without the loss of your research credit. If you withdraw from the study at any time, any data that you have contributed will be destroyed.

Questions: If you have any questions concerning the study, please feel free to ask at any point; you are also free to contact the researchers at the numbers provided above if you have questions at a later time. This study has been approved on ethical grounds by the University of Saskatchewan Behavioural Research Ethics Board on (Oct. 26, 2007). Any questions regarding your rights as a participant may be addressed to that committee through the Ethics Office (966-2084). Out of town participants may call collect. You may contact the researcher if you wish to obtain the results of the study.

Consent to Participate: I have read and understood the description provided above; I have been provided with an opportunity to ask questions and my questions have been answered satisfactorily. I consent to participate in the study described above, understanding that I may withdraw this consent at any time. A copy of this consent form has been given to me for my records.

(Name of Participant)

(Signature of Participant)
(Date)

(Signature of Researcher) 


\title{
APPENDIX N \\ DEBRIEFING FORM FROM PERCEPTUAL ASYMMETRIES IN GREYSCALES: OBJECT- BASED VERSUS SPACE-BASED INFLUENCES (STUDY 1)
}

\author{
Study Title: Upper and Lower Visual Field Differences in Pseudoneglect (F34)
}

Thank-you for participating in the study!

It has been well established that normal people tend to underestimate the right side when judging distance judgment or bisecting lines. It is also true that when asked, participants select images that are lit from the left as being either brighter or darker. You completed a computer task where you were asked to select which image appeared darker. The images that you viewed were of equal luminance; however most people select the image with salient feature (i.e., dark) located on the left side as being darker overall.

A few studies have examined differences in line bisection related to where the line is located in space. It has been found that participants will bisect the same line in a different location depending on whether the line is located above or below eye-level. Some investigators have found that bisections are more leftward above eye-level, but others have found that they are more leftward below eye-level.

These differences might also interact with differences between the left side of space and the right side of space. You viewed a pair of images, where sometimes one image was directly on top of the other one, other times the images were slightly overlapped, and other times the images did not overlap at all. It is thought that the common leftward bias might depend on where the image is in space (i.e., top left, top right, bottom left, bottom right).

This leftward bias may be due to activation of the right hemisphere during spatial tasks, scanning related to reading habits (left-to-right), lateral biases in attention or perception, or a pre-motor bias related to the hand that is used to perform the task. The current study should help to explain this common leftward bias. 


\section{APPENDIX O}

STIMULI USED IN PERCEPTUAL ASYMMETRIES IN GREYSCALES: OBJECT-BASED VERSUS SPACE-BASED INFLUENCES (STUDY 2)
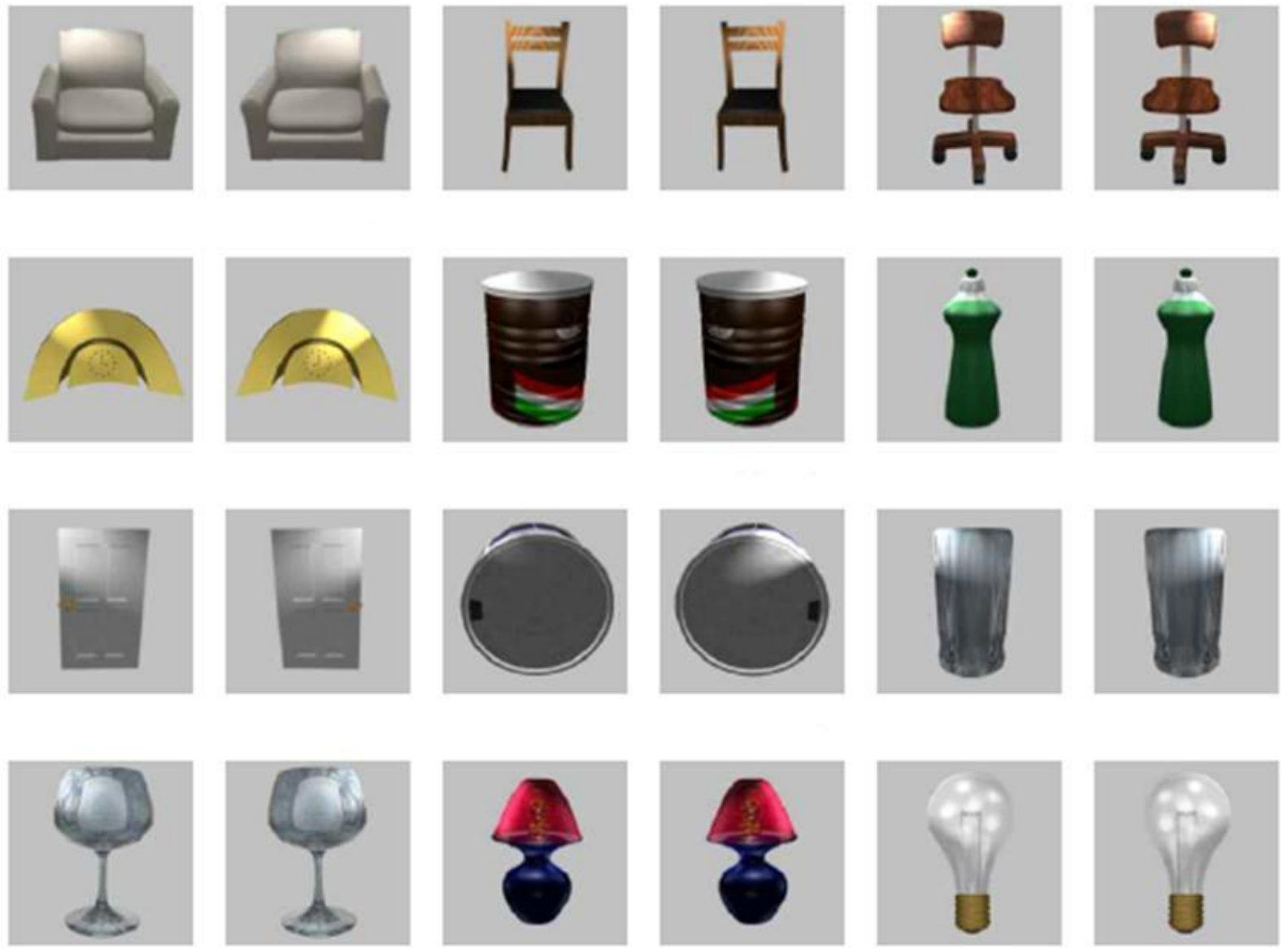


\title{
APPENDIX P \\ CONSENT FORM FROM PERCEPTUAL ASYMMETRIES IN GREYSCALES: OBJECT- BASED VERSUS SPACE-BASED INFLUENCES (STUDY 2)
}

\author{
Judging Brightness \\ Researcher: Dr. L. Elias, Department of Psychology \\ Dr. D. Saucier, Department of Psychology
}

Purpose and objectives of the study: Previous studies have suggested that people judge the brightness of objects differently depending on where they are presented on a computer screen. This study is meant to test whether this is the case. Further, we will also be testing whether the differences are present when examining pictures of real objects.

Possible benefits of the study: This project should help clarify whether the differences in how people make these judgments actually exist. Participants will gain experience with experimental psychology, and if they wish, they will be given opportunity to learn of the results of the study (only group results will be released). Each participant will receive one credit for their participation.

Procedure: Participants will complete a brief demographic questionnaire, followed by the following task: making judgements about the brightness of various objects. The entire session should take less than $1 / 2$ hour.

Possible Risks: There are no known risks associated with this procedure.

I, , have read the above description and agree to participate. The procedure and its possible risks have been explained to me by the researcher, and I understand them. I understand that I am free to withdraw from this study at any time without penalty of any type and I will not loose credit for the session. I also understand that although the data from this study may be published in a research article, only aggregate data will be described and that my identity will be kept confidential. I also confirm that I have received a copy of this consent form for my records.

(Signature)

(Date)

(Researcher)

Department of Psychology

University of Saskatchewan 


\section{APPENDIX Q \\ DEBRIEFING FORM FROM PERCEPTUAL ASYMMETRIES IN GREYSCALES: OBJECT- BASED VERSUS SPACE-BASED INFLUENCES (STUDY 2)}

\section{Judging Brightness}

Thank you for participating in the study!

Previous studies have shown that people judge objects differently depending on which side of a computer screen they are presented on. One way of testing this effect is by presenting two identical objects on each side of the screen and asking people top make judgements about the objects. For example, when identical (but mirrored) objects are presented on each side of the screen and people are asked to indicate which object is darker, they usually (66\% of the time) indicate that the object on the left is darker. Similarly, when identical (but mirrored) objects are presented on both sides of the screen and people are asked to indicate which object is bigger, they usually choose the object on the left.

The cause of this strange effect is unknown. One possibility is that people tend to scan the objects from left to right (as in normal reading). Another possibility is that people pay more attention to complex visual objects on their left side.

You were presented with a number of pairs of different objects. In some cases, there was a difference in brightness between the objects. On other occasions, the two objects were identical. We predicted that people would be more likely to choose the object wherein the leftward side of the object contained the brighter region, even if the two objects were identical in brightness overall. 


\section{APPENDIX R \\ DEBRIEFING FORM FROM UPPER AND LOWER VISUAL FIELD DIFFERENCES IN PERCEPTUAL ASYMMETRIES}

\section{Study Title: Upper and Lower Visual Field Differences in Pseudoneglect (F34)}

Thank-you for participating in the study!

It has been well established that normal people tend to underestimate the right side when judging distance judgment or bisecting lines. It is also true that when asked, participants select images that are lit from the left as being either brighter or darker. You completed a computer task where you were asked to select which image appeared darker. The images that you viewed were of equal luminance; however most people select the image with salient feature (i.e., dark) located on the left side as being darker overall.

A few studies have examined differences in line bisection related to where the line is located in space. It has been found that participants will bisect the same line in a different location depending on whether the line is located above or below eye-level. Some investigators have found that bisections are more leftward above eye-level, but others have found that they are more leftward below eye-level.

These differences might also interact with differences between the left side of space and the right side of space. You viewed a pair of images, where sometimes one image was directly on top of the other one, other times the images were slightly overlapped, and other times the images did not overlap at all. It is thought that the common leftward bias might depend on where the image is in space (i.e., top left, top right, bottom left, bottom right).

This leftward bias may be due to activation of the right hemisphere during spatial tasks, scanning related to reading habits (left-to-right), lateral biases in attention or perception, or a pre-motor bias related to the hand that is used to perform the task. The current study should help to explain this common leftward bias. 


\section{APPENDIX S}

SAMPLE STIMULI FROM THE RED-GREEN ISOLUMINANT COLOUR TASK 


\section{APPENDIX T \\ CONSENT FORM FROM DORSAL AND VENTRAL STREAM CONTRIBUTIONS TO PERCEPTUAL ASYMMETRIES}

You are invited to participate in a study entitled Detecting Motion and Luminance (Q6). Please read this form carefully, and feel free to ask questions you might have.

Researchers: Nicole Thomas, Department of Psychology, University of Saskatchewan

Dr. Lorin Elias, Department of Psychology, University of Saskatchewan

Purpose and Procedure: The purpose of this study is to investigate judgments of luminance and motion.

Participants will complete six computer tasks where they will be asked to make judgments of luminance as well as complete a demographic questionnaire, which will be used to describe the general characteristics of the entire group of participants. This study will take approximately 30 minutes.

Potential Risks: There are no known risks associated with this procedure; however you may receive no personal benefit from participation in the study. At the end of the study you will be given a sheet that better explains the nature of the study and you will be given a chance to ask any questions you might have.

Potential Benefits: This study should lead to further understanding of differences in judgments of luminance and motion as they relate to the visual system. As well, this study should help to understand how these particular judgments relate to the way the brain functions. Participants will gain experience with experimental psychology, and if they wish, they will be given the opportunity to learn the group results of the study.

Storage of Data: The data and consent forms will be stored securely at the University of Saskatchewan by Dr. L. Elias. Consent forms will be stored separately from the data materials so that it will not be possible to associate names with any given data. The data will be stored for five years after completion of the study and then destroyed beyond recovery.

Confidentiality: Your data will be kept completely confidential and no personally identifying information will be linked to your data. All data will be reported in aggregated form.

Right to Withdraw: Your participation is voluntary, and you may withdraw from the study for any reason, at any time, without penalty of any sort and without the loss of your research credit. If you withdraw from the study at any time, any data that you have contributed will be destroyed beyond recovery.

Questions: If you have any questions concerning the study, please feel free to ask at any point; you are also free to contact the researchers at the numbers provided above if you have questions at a later time. This study has been approved on ethical grounds by the University of Saskatchewan Behavioural Research Ethics Board on (Oct. 26, 2007). Any questions regarding your rights as a participant may be addressed to that committee through the Ethics Office (966-2084). Out of town participants may call collect. You may contact the researcher if you wish to obtain the results of the study.

Consent to Participate: I have read and understood the description provided above; I have been provided with an opportunity to ask questions and my questions have been answered satisfactorily. I consent to participate in the study described above, understanding that I may withdraw this consent at any time. A copy of this consent form has been given to me for my records.

(Name of Participant)

(Signature of Participant)
(Date)

(Signature of Researcher) 


\title{
APPENDIX U \\ DEBRIEFING FORM FROM DORSAL AND VENTRAL STREAM CONTRIBUTIONS TO PERCEPTUAL ASYMMETRIES
}

\author{
Study Title: Detecting Motion and Luminance (Q6)
}

Thank-you for participating in the study!

It has been well established that the majority of people tend to underestimate the right side when making luminance judgments or bisecting lines. It is also true that participants tend to select images that are lit from the left as being either brighter or darker than identical but mirrorreversed images. You completed a computer task where you were asked to select which image appeared darker. The images that you viewed were of equal luminance; however most people select the image with the dark portion located on the left side as being darker overall.

A few studies have examined differences in line bisection related to where the line is located in space. It has been found that participants will bisect the same line in a different location depending on whether the line is located above or below eye-level. Some investigators have found that bisections are more leftward above eye-level, but others have found that they are more leftward below eye-level. Similar results are found when individuals are asked whether a line that is already bisected is bisected to the left or the right of the middle of the line. Despite these mixed findings, it is clear that differences exist depending on the location of the stimulus.

Some researchers suggest that these differences are related to information processing differences between the two visual streams in the brain: the ventral stream and the dorsal stream. The ventral visual stream is thought to be linked with processing information in above eye-level, whereas the dorsal visual stream is related to information processing below eye-level. It is also known that the dorsal visual stream is better at perceiving motion, whereas the ventral visual stream is more involved in perceiving colour. The current study should increase understanding of these differences as well as understanding of how the human visual system perceives information. 


\section{APPENDIX V}

SAMPLE STIMULI FROM THE BLUE-YELLOW ISOLUMINANT COLOUR TASK

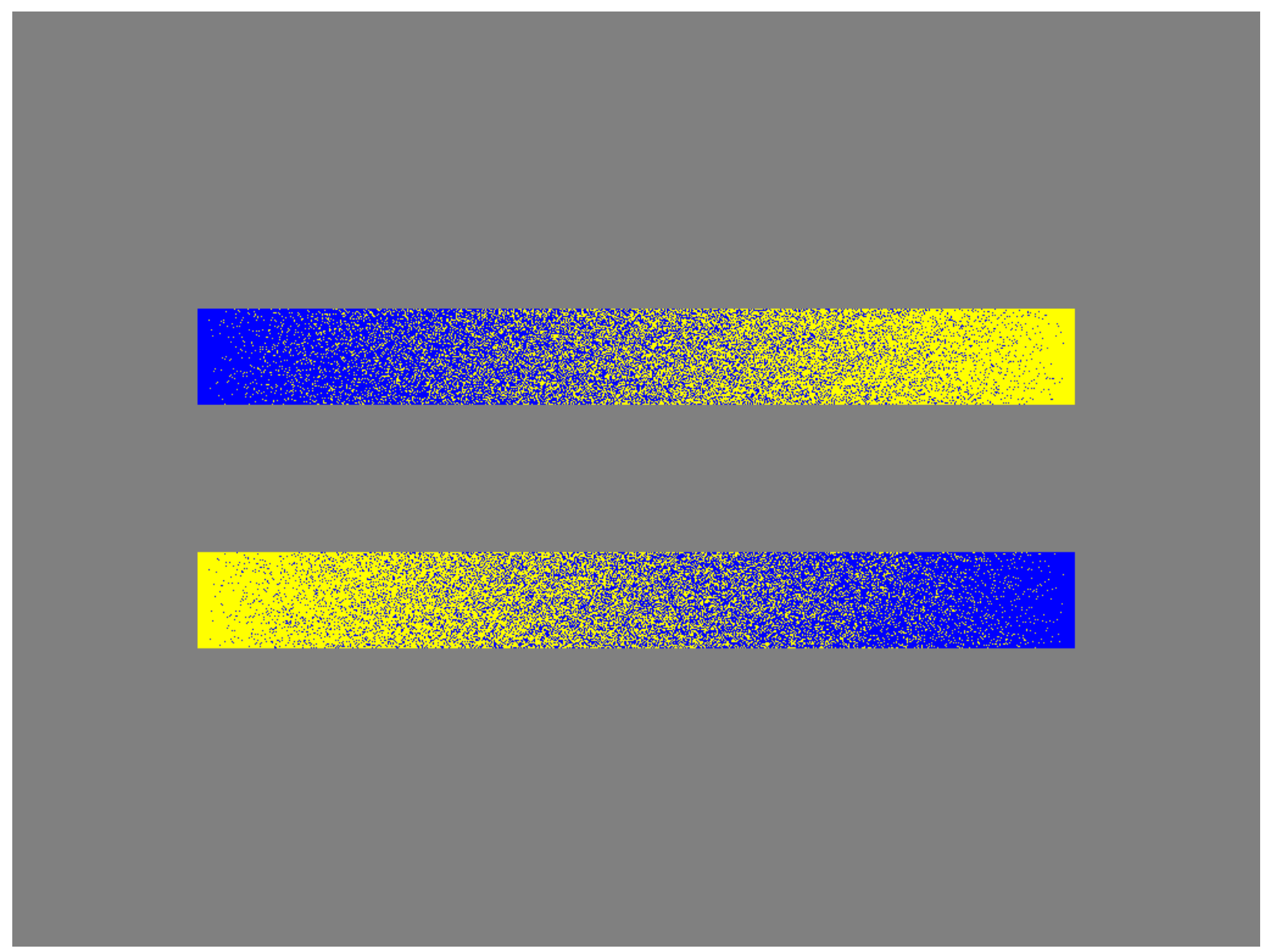

